\title{
Wölbtorsionsversuche an Stahlbetonbalken mit offenem Querschnitt
}

\section{Working Paper}

\section{Author(s):}

Grob, Josef; Thürlimann, Bruno

Publication date:

1974

\section{Permanent link:}

https://doi.org/10.3929/ethz-a-002213789

\section{Rights / license:}

In Copyright - Non-Commercial Use Permitted

\section{Originally published in:}

Bericht / Institut für Baustatik und Konstruktion ETH Zürich 6506(6) 
Wölbtorsionsversuche an Stahlbetonbalken mit offenem Querschnitt

Josef Grob

Bruno Thürlimann 


\title{
Wölbtorsionsversuche an Stahlbetonbalken mit offenem Querschnitt
}

\author{
von \\ Dipl. Ing. Josef Grob \\ Prof. Dr. Bruno Thürlimann \\ Institut für Baustatik \\ Eidgenössische Technische Hochschule Zürich
}


1. EINLEITUNG 1

1.1 Problemstellung und Zielsetzung

1.2 Versuchsprogramm

2. VERSUCHSBALKEN 2

2.1 Beschreibung 2

2.2 Baustoffe 3

2.3 Rechnerische Werte 4

3. VERSUCHSDURCHFUEHRUNG

3.1 Versuchsanlage 9

3.2 Versuchsablauf 9

4. VERSUCHSRESULTATE 11

4.1 Trag - und Bruchverhalten 11

4.2 Beanspruchung der Armierung 14

4.3 Beanspruchung des Betons 16

4.4 Verformungen 17

4.5 Rissverhalten 18

ZUSAMMENFASSUNG $\quad 20$

RESUME $\quad 21$

SUMMARY 22

VERDANKUNGEN

LITERATURVERZEICHNIS $\quad 24$

BEZEICHNUNGEN $\quad 25$

TABELLEN 1 BIS $9 \quad 30$

BILUER 1 BIS 52

ANHANG A1 


\section{EINLEITUNG}

\subsection{Problemstellung und Zielsetzung}

Das Verhalten von Stahlbeton- und Spannbetonbalken mit volien oder geschlossenen Querschnitten ist bisher experimentell eingehend untersucht worden. Am Institut für Baustatik, Abt. Massivbau, der Eidgenössischen Technischen Hochschule (ETH) in Zürich erschienen darüber die vier Berichte [2], [3], [4] und [5]. Für Balken mit offenen Querschnitten hingegen fehlen entsprechende Untersuchungen, obwohl in jüngster Zeit die Anwendung offener Querschnitte eine starke Verbreitung erfahren hat (offene Brücken, Kerne von Hochhäusern, vorfabrizierte Dachschalen, etc.). Aus diesem Grund läuft zur Zeit am Institut für Baustatik der Eidgenössischen Technischen Hochschule (ETH) in Zürich ein Forschungsprogramm, welches sich mit dem Verhalten von Stahlbetonund Spannbetonbalken mit offenen Querschnitten befasst.

Das Ziel dieses Programms besteht darin, ein theoretisches Modell für die Berechnung von Stahlbeton- und Spannbetonbalken mit offenen Querschnitten zu entwickeln und durch gezielte Versuche zu überprüfen.

\subsection{Versuchsprogramm}

Für das vorliegende Versuchsprogramm wurde ein $\dot{7}$-Querschnitt mit vernünftig grossen Abmessungen gewählt. Als Grundform drängte sich ein $\longrightarrow$ mit konstanten Seitenlängen von $50 \mathrm{~cm}$ auf, um Teile der in [1] beschriebenen Versuchsanlage verwenden zu können. Das experimentelle Versuchsprogramm ist in Bild 1 zusammengestellt.

Es wurde versucht, mit mäglichst wenig Aufwand eine aussagekräftige Parametervariation zu erhalten. Um gute Vergleichsmäglichkeiten zu schaffen, wurden die Versuchsbalken so gewählt, dass zwischen zwei Balken $A$ und $B$ jeweils nur ein Parameter änderte. Dies dokumentiert die nachstehende Zusammenstellung.

Balken A

$\mathrm{TW}$
$\mathrm{TW}$
$\mathrm{TW}$
$\mathrm{T}_{3}$

Balken $B$

$\mathrm{TW}_{3}$

$\mathrm{TW}_{2}$

$\mathrm{TW}_{4}$

$\mathrm{TW}_{5}$
Parametervariation

Beanspruchung

Längsarmierung

Schubarmierung

Vorspannung 


\section{VERSUCHSBALKEN}

\subsection{Beschreibung}

\subsubsection{Abmessungen und Armierung}

Abmessungen und Armierung der Versuchsbalken gehen aus Bild 2 hervor. Alle Balken haben den gleichen relativ gedrungenen Beton-Querschnitt. Die gewählte Wandstärke von $8 \mathrm{~cm}$ ermäglichte eine einwandfreie kontruktive Ausbildung der Versuchskörper sowie die Verwendung eines Betons mit einem vernünftigen Maximalkorndurchmesser.

Mit Ausnahme der beim Balken $T_{5}$ verwendeten Vorspannlitzen (VSL) bestand die gesamte Armierung aus naturhartem profiliertem Stahl (Box-Ultra, bzw. Baro). Die Bügel wurden mit einem Abbiegeradius von $2 \mathrm{~cm}$ gebogen und deren Enden zwecks Gewährleistung der Verankerung angeschweisst. Die verlangte Abbiegetoleranz der Bügelabmessungen von $\pm 2 \mathrm{~mm}$ wurde singehalten. Die Betonüberdeckung der Bügel betrug bei den Balken TW, bis $T_{3} 13 \mathrm{~mm}$, diejenige der Balken $T_{4}$ und $T W_{5} 15 \mathrm{~mm}$. Somit ergab sich für die Längseisen bei allen Balken eine Ueberdeckung von mehr als $20 \mathrm{~mm}$.

Die Krafteinleitungsstellen an den Balkenenden wurden durch zusätzliche Stahleinlagen verstärkt, um einen Bruch in diesem Bereich zu vermeiden. Zudem wurden füp die massgebenden Längseisen gegen die Balkenmitte hin grössere Durchmesser gewählt. Der Uebergang zwischen den beiden Durchmessern wurde aus Platzgründen stumpf verschweisst. In Balkenmitte wurde ein $20 \mathrm{~cm}$ breiter Querträger angeordnet, um eine saubere krafteinleitung zu erhalten. Der eigentliche Prüfbereich von $60 \mathrm{~cm}$ Länge wurde zwischen den Krafteinleitungszonen angeordnet.

\subsubsection{Herstellung}

Als Schalung wurde eine Holzschalung verwendet, die mit einer Kunststoffplatte überzogen war, um ein Verziehen zu verhindern. Der Beton wurde in einem 500 Liter Zwangsmischer in einer Mischcharge von 550 Liter je Träger hergestellt und nach dem Einbringen mit Nadelvibratoren $\oslash 40 \mathrm{~mm}$ verdichtet. Um dies zu ermöglichen, wurden die oberen Längseisen erst nach dem Betonieren der beiden Balkenstege eingezogen. Nach erfolgtem Glattstrich schützten Plastikfolien den frischen Beton vor dem Austrocknen. Das Ausschalen erfolgte nach zwei Tagen, worauf die Balken bis zum Versuch bei konstanter Raumtemperatur von ungefähr $20^{\circ}$ C lagerten. Die Vorbereitung der Stahlmessstellen ist im Bericht [2] beschrieben.

Der Balken TW 5 wurde nach 31 Tagen (1 Woche vor Versuchsbeginn) vorgespannt und gleich darauf injiziert. Die Grösse der Vorspannung geht aus bild 6 hervor und wurde mit einem Präzisionsmanometer kontrolliert. 


\subsection{Baustoffe}

\subsubsection{Schlaffer Stah1}

Die Festigkeitswerte der verwendeten Armierungsstähle gehen aus Tabelle 1 hervor. Sie sind für jeden der verwendeten Durchmesser getrennt aufgeführt, da die gemessenen Streuungen für Stähle desselben Durchmessers klein und die Mittelwerte folglich repräsentativ sind. Ein typisches, auf einer mechanischen Prüfmaschine aufgenommenes Spannungs-Dehnungs-Diagramm ist in Bild 3 dargestellt.

Für die Auswertung wurden die gemittelten idealisierten Diagramme von Bild 4 verwendet.

Da die dynamischen Festigkeitswerte von der Dehnungsgeschwindigkeit $\dot{\varepsilon}=d \varepsilon / d t$ abhängig sind, wurden die statischen Werte $(\dot{\varepsilon}=0)$ der Auswertung zugrunde gelegt. Sie wurden durch zweiminütige Dehnungshalte beim Fliessplateau und bei der Bruchgrenze ermittelt. Einzelheiten dieses Verfahrens sind in [6] enthalten. Das SpannungsDehnungs-Diagramm wurde so dem Ablauf der Balkenversuche angepasst.

\subsubsection{Spannstahl}

Die Festigkeitswerte der verwendeten Litzen sind in Tabelle 1 ersichtlich. Bild 5 zeigt ein typisches Spannungs-Dehnungs-Diagramm einer solchen Litze. Der Auswertung liegt das gemittelte Diagramm von Bild 6 zugrunde, wobei analog dem schlaffen Stahl die statischen Festigkeitswerte berücksichtigt wurden.

\subsubsection{Beton}

Die Zusammensetzung des Betons war für alle Balken gleich und ist in Tabelle 2 angegeben. Der Zuschlagstoff, der aus gut gewaschenem und getrocknetem, rolligem Material bestand, wurde getrennt in drei Komponenten beigegeben. Die Sieblinie entsprach ungefähr der EMPA-Kurve. Als Zement wurde schweizerischer Portlandzement verwendet. Seine Mörtelfestigkeit wurde nicht bestimmt.

Zur Ermittlung der Betonfestigkeit wurden gleichzeitig mit jedem Balken sechs Prismen $(12 \times 12 \times 36 \mathrm{~cm})$ hergestellt. Im Versuchsalter der Balken wurden jeweils an zwei Prismen die Prismendruckfestigkeit sowie der Elastizitätsmodul und an weiteren drei Prismen die Biegezug- und Würfeldruckfestigkeit ermittelt. Diese Werte wurden bei relativ hoher Dehngeschwindigkeit gemessen und sind in Tabelle 3 festgehalten.

Ergänzend wurde je an einem Prisma pro Balken mit einer mechanisch-elektrischen Messeinrichtung, welche in [2] beschrieben ist, ein dehnungsgesteuertes Spannungs-Dehnungs-Diagramm aufgenommen. Als Beispiel wird in Bild 7 das Diagramm des zum Balken $\mathrm{TW}_{2}$ gehörenden Prismas aufgeführt. Tabelle 4 gibt eine Vebersicht über alle mit diesen Versuchen erhaltenen Werte.

Für die Auswertung der in den Balkenversuchen beobachteten Betondehnungen wurden die effektiven Spannungs-Dehnungs-Diagramme durch die kubische Parabel

$$
\frac{\sigma_{b}}{\beta_{p}}=\rho \cdot\left(\frac{\varepsilon_{b}}{\varepsilon_{u}}\right) \cdot\left[1-\left(2-\frac{3}{\rho}\right) \cdot\left(\frac{\varepsilon_{b}}{\varepsilon_{u}}\right)+\left(1-\frac{2}{\rho}\right) \cdot\left(\frac{\varepsilon_{b}}{\varepsilon_{u}}\right)^{2}\right]
$$


mit

$$
\rho=\varepsilon_{u} \cdot \frac{E_{b 0}}{\beta_{p}} \text { gültig für } 1,5<\rho<3,0
$$

angenähert ( $v g l$. Bild 7). Dabei wurden für $\beta_{p}$ und $\varepsilon_{u}$ die aus der Tabelle 4 entnommenen Mittelwerte eingesetzt; $E_{b 0}$ wurde so gewählt, dass der Mittelwert für $E_{b(5 \div 100)}$ mit dem entsprechenden Wert der theoretischen Kurve übereinstimmt. Da die statischen Festigkeitswerte $(\dot{\varepsilon}=0)$ infolge Kriechen von der Dauer des Dehnungshaltes abhängig sind, wurde wie in [2] bis [4] die einer 30-minütigen Wartezeit entsprechende Prismendruckfestigkeit berücksichtigt. Damit wurden die Spannungs-Dehnungs-Diagramme dem Ablauf der Balkenversuche angepasst und der Einfluss des Kriechens während der Dehnungshalte miteinbezogen. Die nach Gleichung (1) berechneten Diagramme für Dehnungshalte von 2 resp. 30 Minuten sind in Bild 8 wiedergegeben.

\subsection{Rechnerische Werte}

\subsubsection{Ungerissener Zustand}

Die am ungerissenen Querschnitt eingeführten Bezeichnungen sind in Bild 9 dargestellt. Solange der Träger keine Risse aufweist, finden die Beziehungen der Elastizitätstheorie Anwendung. Trotz gedrungenem Querschnitt werden im folgenden die Gleichungen für dünnwandige Querschnitte verwendet, da für den ungerissenen Zustand nur Vergleichsspannungen bei Rissbeginn berechnet und Vergleiche mit der Biegezugfestigkeit des Betons angestellt werden, welche bekanntlich eine grosse Streuung aufweist. Ferner sind das hauptaxensystem sowie die Lagen des Schwer- und des Schubmittelpunktes auf den Betonquerschnitt allein bezogen.

Mit den genannten Vereinfachungen erhält man folgende Ausdrücke für die Querschnittskonstanten:

$$
\left.\begin{array}{ll}
\text { Querschnittsfläche } & F=F_{b}+n \cdot F_{e} \\
\text { Trägheitsmoment } & I_{z}=\int_{z_{b}} z^{2} \cdot d F+n \cdot \int_{z^{2}} \cdot d F \\
\text { Wölbkonstante } & I_{\omega}=\int_{F_{b}} \omega_{n}^{2} \cdot d F+n \cdot \int \omega_{n}^{2} \cdot d F \\
\text { Torsionskonstante } & K=\left(2 h_{m}+b_{m}\right) \cdot \frac{t^{3}}{3}
\end{array}\right\}
$$

Mit $n$ wird in den obigen Beziehungen die Wertigkeit $n=E_{e} / E_{b}=7,5$ bezeichnet. Die numerischen Werte für die Querschnittskonstanten sind in Tabelle 5 festgehalten. Wie aus der Elastizitätstheorie bekannt, können die Verschiebung $w$ und die Verdrehung $\vartheta$ aus den folgenden unabhängigen Differentialgleichungen bestimmt werden:

$$
\frac{d^{4} w}{d x^{4}}=\frac{P z}{E I_{z}}
$$


$\left.\begin{array}{cc} & \frac{d^{4} \vartheta}{d x^{4}}-k^{2} \cdot \frac{d^{2} \vartheta}{d x^{2}}=\frac{m_{x}}{E I_{\omega}} \\ \text { mit } \quad & k=\sqrt{\frac{G K \cdot I^{2}}{E I_{\omega}}} \simeq 3,20(G / E=0,40)\end{array}\right\}$

Dabei bedeuten $p_{z}$ die Belastung in Richtung der $z$-Axe und $m_{x}$ die auf den Schubmittelpunkt resuzierte Torsionsbelastung. Die Schnittkräfte sind Funktionen der aus den Gleichungen (4) und (5) bestimmten Verschiebungen $w$ und $\vartheta$.

$\left.\begin{array}{ll}\text { Biegemoment } & M_{z}=-E I_{z} \cdot \frac{d^{2} w}{d x^{2}} \\ \text { Querkraft } & Q_{z}=\frac{d M_{z}}{d x} \\ \text { Wölbmoment } & M_{\omega}=-E I_{\omega} \cdot \frac{d^{2} \vartheta}{d x^{2}} \\ \text { Wälbtorsionsmoment } & T_{w}=\frac{d M_{\omega}}{d x} \\ \text { St. Venant'sches } & \\ \text { Torsionsmoment } & T_{s}=G K \cdot \frac{d \vartheta}{d x}\end{array}\right\}$

Die aus der Einzellast $P$ resultierenden Schnittkräfte. sind in der linken Hälfte des Bildes 14 eingezeichnet. Zudem findet man in Tabelle 6 die im Querschnitt B-B auftretenden Schnittkräfte infolge Eigengewicht. Für eine Spannungsanalyse benötigt man noch die aus der Elastizitätstheorie bekannten Beziehungen für die Längsspannungen

$$
\sigma=\frac{N}{F}+\frac{M_{z}}{I_{z}} \cdot z+\frac{M_{\omega}}{I_{\omega}} \cdot \omega_{n}
$$

und die Schubspannungen

$$
\begin{aligned}
& \tau=\frac{Q_{z} \cdot S_{z}}{I_{z} \cdot t}+\frac{T_{w} \cdot S_{\omega}}{I_{\omega} \cdot t}+\frac{T}{K} \cdot t \\
& \text { mit } \\
& S_{z}=\int_{S} z \cdot d F \\
& S_{\omega}=\int_{s} \omega_{n} \cdot d F
\end{aligned}
$$

Bei Rissbeginn erhält man erfahrungsgemäss bessere Resultate, wenn für die Verteilung der Schubspannungen über die Querschnittsdicke $t$ die Sandhügelanalogie herangezogen wird. Damit ändert sich Gleichung (8) zu:

$$
\tau=\frac{Q_{z} \cdot S_{z}}{I_{z} \cdot t}+\frac{T_{w} \cdot S_{w}}{I_{w} \cdot t}+\frac{2}{3} \cdot \frac{T^{T}}{K} \cdot t
$$

Eine Herleitung der angegebenen Beziehungen findet man in [7] oder [8]. 


\subsubsection{Bruchzustand}

Eine theoretische Untersuchung über das Bruchverhalten von offenen Stah1- und Spannbetonträgern ist in [9] eingehend erläutert. Hier werden nur die nötigen Grundgleichungen sowie die daraus resultierenden Beziehungen für die Versuchskörper angegeben. Es sei an dieser Stelle noch auf den Anhang des Versuchsberichtes hingewiesen, wo eine einfache Berechnungsmethode hergeleitet wird.

Neben den Bezeichnungen zeigt Bild 10 die für die rechnerische Erfassung des Bruchwiderstandes eingeführte Idealisierung des Querschnitts. Das dargestellte Bruchmodell ist ein reines Scheibenmodell, d.h. die Plattenwirkung wird für die theoretische Betrachtung vernachlässigt. Im oberen Teil des Bildes sind die in Tabelle 5 angegebenen Abmessungen, die Fliesskräfte der Armierung sowie das gewählte Koordinatensystem (Ursprung der Querschnittskoordinaten und Bezugspunkt der sektoriellen Koordinate im Punkt 4) eingetragen. Die Breite b bezeichnet den mittleren Abstand der in den Scheiben 2 und 6 wirkenden Schubflüsse. Als Höhe h wird der SchwerpunktsAbstand der Gurtstäbe angenommen.

Die Schnittkräfte sind durch die Gleichgewichtsbedingungen (10) und (11) gegeben.

$$
\left.\begin{array}{l}
\frac{d Q_{z}}{d x}+p_{z}=0 \\
\frac{d^{2} M_{z}}{d x^{2}}+p_{z}=0
\end{array}\right\}
$$

und

$$
\left.\begin{array}{l}
\frac{d T_{w}}{d x}+m_{x}=0 \\
\frac{d^{2} M_{\omega}}{d x^{2}}+m_{x}=0
\end{array}\right\}
$$

Dabei sind die Belastung $p_{z}$ und $m_{x}$ wie die Schnittkräfte auf den Punkt 4 zu beziehen. Auffallend ist die zwischen den Gleichungen (10) und (11) bestehende Analogie. Danach lassen sich die Schnittkräfte $T_{w}$ und $M_{\omega}$ nach denselben Methoden bestimmen wie die von der Balkenbiegung her geläufigen Schnittkräfte $Q_{z}$ und $M_{z}$. Der Verlauf der aus der Last $P$ resultierenden Schnittkräfte ist in der rechten Hälfte des Bildes 14 eingetragen.

Gemäss den Zeichnungen in Bild 10 wird für die Einzelscheibe ein Fachwerkmodell mit variabler Neigung der Druckdiaganalen angenommen. Das Bruchmodell besteht dann aus einem räumlichen Fachwerk mit variabler Diagonalenneigung $\alpha_{k}$. In ihm bilden die Längseisen die Gurtungen, die Bügel die Pfosten und die Betondiagonalen die Druckstreben. Die Ausbildung eines solchen Fachwerkes verlangt genau wie bei den geschlossenen Querschnitten eine genügend starke Längsarmierung in den Querschnittsecken, um ein Ausbrechen der Druckdiagonalen zu verhindern. Für den untersuchten Querschnitt ist das Kräftespiel in der unteren Bildhälfte dargestellt. Es gelten die folgenden Gleichgewichtsbedingungen: 


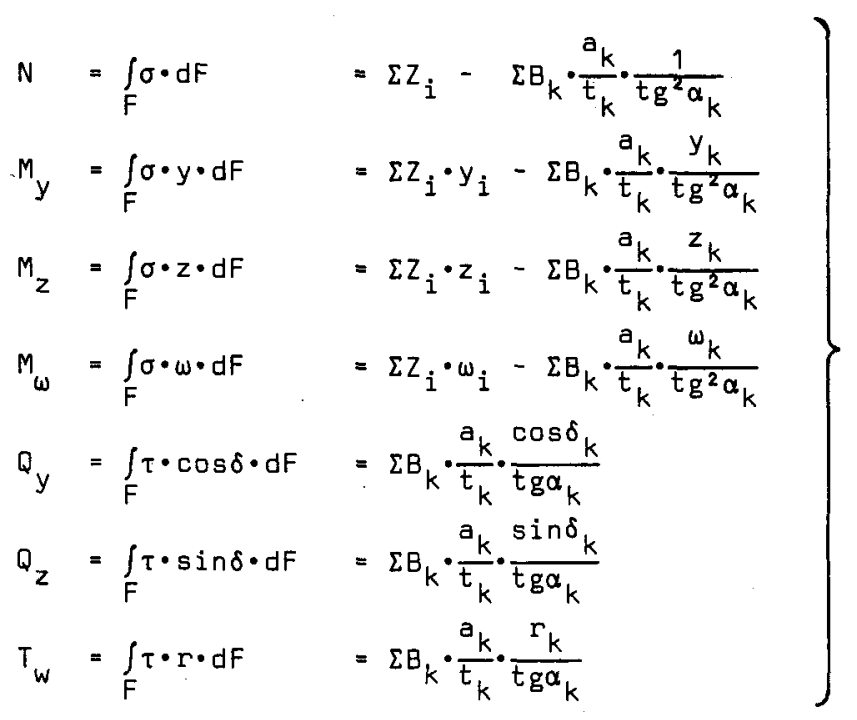

Mit $r$ wird hier der Abstand der Querschnittstangente und mit $r_{k}$ der Abstand der k-ten Querschnittscheibe vom Punkt 4 bezeichnet, auf den die Schnittkräfte $T_{w}$ und $M_{\omega}$ bezogen sind. Als $\delta$ wird der Winkel zwischen y-Axe und Querschnittstangente und als $\delta_{k}$ der Winkel zwischen $y$-Axe und k-ter Querschnittscheibe eingeführt.

Das Gleichungssystem (12) liefert für $N=Q_{y}=M_{y}=0$, wie dies für alle Versuchsbalken zutrifft, die Beziehungen für die in den Bügeln und Gurtstäben wirkenden kräfte.

$$
\begin{aligned}
& B_{2} \cdot \frac{h}{t_{B}} \cdot \frac{1}{\operatorname{tg} \alpha_{2}}=Q_{2}=-\frac{Q_{z}}{2}+\frac{T_{w}}{b} \\
& B_{4} \cdot \frac{b}{t_{B}} \cdot \frac{1}{\operatorname{tg} \alpha_{4}}=Q_{4}=0 \\
& B_{6} \cdot \frac{h}{t_{B}} \cdot \frac{1}{\operatorname{tg} \alpha_{6}}=Q_{6}=\frac{Q_{z}}{2}+\frac{T_{w}}{b} \\
& z_{1}=\frac{M_{z}}{2 h}-\frac{M_{w}}{b \cdot h}+\frac{1}{2} \cdot\left(-\frac{Q_{z}}{2}+\frac{T w}{b}\right) \cdot \frac{1}{\operatorname{tg} \alpha_{2}} \\
& z_{3}=-\frac{M_{z}}{2 h}+\frac{M_{\omega}}{b \cdot h}+\frac{1}{2} \cdot\left(-\frac{Q_{z}}{2}+\frac{T w}{b}\right) \cdot \frac{1}{\operatorname{tg} \alpha_{2}} \\
& z_{5}=-\frac{M_{z}}{2 h}-\frac{M_{w}}{b \cdot h}+\frac{1}{2} \cdot\left(\frac{Q_{z}}{2}+\frac{T w}{b}\right) \cdot \frac{1}{\operatorname{tg} \alpha_{6}} \\
& z_{7}=\frac{M_{z}}{2 h}+\frac{M_{w}}{b \cdot h}+\frac{1}{2} \cdot\left(\frac{Q_{z}}{2}+\frac{T w}{b}\right) \cdot \frac{1}{t_{g} \alpha_{6}}
\end{aligned}
$$

Bei unterarmierten Trägern wird ein Mechanismus durch Stahlfliessen verursacht, d.h. die Armierung wird für die Bruchlast massgebend. Wie in den Bildern 11 bzw. 12 dargestellt ist, kann sich für die untersuchten Balken ein Mechanismus ausbilden, wenn entweder die Balkenvorderseite oder die Balkenhinterseite versagt. Bei einem solchen Versagen kommen innerhalb gewisser Grenzen dank der sich einstellenden Umlagerung des Winkels a sowohl Bügel-als auch Längsarmierung zum Fliessen. Zu erwähnen ist noch, dass in den schematischen Darstellungen der Bilder 11 und 12 nur die plastischen Verformungen berücksichtigt sind, d.h. die vorangegangenen elastischen Verformungen sind in diesen bildern nicht eingetragen. 
Beim Versagen der Balkenvorderseite, wie dies bei den Balken $T W_{1}$, $T W_{3}$, $T W_{4}$ und $T W_{5}$ zu beobachten war, müssen die in der Scheibe 6 liegenden Bügel sowie der Gurtstab 7 fliessen.

$$
\begin{aligned}
& \frac{1}{t_{g} \alpha_{6}}=\left(\frac{Q_{z}}{2}+\frac{T_{w}}{b}\right) \cdot\left(B_{f} \cdot \frac{h}{t_{B}}\right)^{-1} \\
& z_{f 7}=\frac{M_{z}}{2 h}+\frac{M_{w}}{b \cdot h}+\frac{1}{2} \cdot\left(\frac{Q_{z}}{2}+\frac{T}{b}\right)^{2} \cdot\left(B_{f} \cdot \frac{h}{t_{B}}\right)^{-1}
\end{aligned}
$$

Die nicht verwendeten Beziehungen aus dem Gleichungssystem (13) dienen lediglich zur Plastizitätskontrolle. Es muss nachgewiesen werden, dass keine in einem Bügel oder Gurtstab wirkende Kraft ihre Fliessgrenze übersteigt.

Versagt hingegen die Balkenhinterseite wie bei $T_{2}$, so müssen der Gurtstab 3 und die Bügel der Scheibe 2 fliessen.

$$
\begin{aligned}
& \frac{1}{t_{g \alpha} \alpha_{2}}=\left(-\frac{Q_{z}}{2}+\frac{T_{w}}{b}\right) \cdot\left(B_{f} \cdot \frac{h}{t_{B}}\right)^{-1} \\
& z_{f 3}=-\frac{M_{z}}{2 h}+\frac{M_{w}}{b \cdot h}+\frac{1}{2} \cdot\left(-\frac{Q_{z}}{2}+\frac{T_{w}}{b}\right)^{2} \cdot\left(B_{f} \cdot \frac{h}{t_{B}}\right)^{-1}
\end{aligned}
$$

Betrachtet man den für den Bruch des Balkens massgebenden Querschnitt-A-A, so erhält man mit den entsprechenden Schnittkräften (siehe Tabelle 6 ind Bild 14 rechts)

$$
\left.\begin{array}{l}
Q_{z}=Q_{z}(g)-0,50 \cdot P \\
T_{w}=T_{w}(g)-0,50 \cdot P \cdot e \\
M_{z}=M_{z}(g)+0,125 \cdot P \cdot 1 \\
M_{\omega}=M_{\omega}(g)+0,125 \cdot P \cdot e \cdot 1
\end{array}\right\}
$$

aus (14b) oder (15b) jeweils eine quadratische Gleichung in $P$. Die positive Wurzel der betreffenden quadratischen Gleichung ergibt dann die theoretische Traglast $P^{T h}$ des angenommenen Scheibenmodells. Die numerischen Werte für $P_{u}^{T h}$ sind in Tabelle 7 angegeben. 


\section{VERSUCHSDURCHFUEHRUNG}

\subsection{Versuchsanlage}

Die Balkenversuche wurden auf dem Aufspannboden in der Prüfhalle der Eidgenössischen Materialprüfungs - und Versuchsanstalt (EMPA) in Dübendorf (ZH) durchgeführt.

Eine Gesamtansicht der Versuchsanlage zeigt Bild 15. Von der in [1] beschriebenen Versuchsanlage konnte der Belastungsrahmen mit Zugpresse verwendet werden. Die Auflagerung der Versuchsbalken hingegen erforderte für das vorliegende Versuchsprogramm eine Ergänzung der bereits bestehenden Prüfeinrichtung. Entsprechend dem auf Bild 13 skizzierten Systemschema musste eine Lagerung gefunden werden, welche weder eine wölbung noch eine Neigung der Balkenenden verhinderte. Dazu wurden zwei Auflagerrahmen konstruiert, von denen der eine in Bild $16 \mathrm{zu}$ sehen ist. Die krafteinleitung vom Auflagerrahmen in den Versuchskörper wurde durch zwei Lager besorgt. Jedes dieser Lager bestand im Prinzip aus einer Kombination eines Teflon-Gleitlagers mit einem Kreuzgelenk, so dass die gewünschten Randbedingungen wenigstens näherungsweise erfüllt werden konnten. Im oberen Lager war die seitliche Bewegungsmöglichkeit auf einige Millimeter beschränkt. Dadurch konnten kleine Horizontalkräfte in der Rahmenebene, wie sie z.B. eine Schiefstellung der Zugpresse verursachte, aufgenommen werden. In Längsrichtung wurde der Versuchsbalken, wenn man von der Schiefstellung der Lager und der Lagerreibung absieht, lediglich durch, die Zugpresse stabilisiert. Um schon bei Versuchsbeginn eine gleichmässige Auflage des Balkens in beiden Auflagerrahmen erreichen zu können, waren die oberen Riegel der Auflagerrahmen senkrecht und die deran befestigten Lager wagrecht verstellbar.

Schliesslich wurde in den Auflagerebenen der Versuchsbalken ein zur Entstehungszeit der Bilder 15 und 16 noch nicht montiertes Gestell angebracht, um die Querschnittsform beim Auflager zu erhalten. Nebenbei kann bemerkt werden, dass es für die Scheibenwirkung eines Dreischeiben-Querschnittes nicht von Bedeutung ist, ob sich der Querschnitt verformen kann oder nicht. Die Plattenwirkung hingegen ist von den Bedingungen abhängig, die an die Verformung des Querschnittes gestellt werden.

\subsection{Versuchsablauf}

Der Versuchsablauf entsprach demjenigen der früheren Torsionsversuche und ist in [2] erläutert. In Bild 17 ist ein Belastungsschema dargestellt. Wesentlich ist, dass während der Verformungsmessungen (Phase II in Bild 18) die Deformation des Balkens konstant gehalten wurde. Die dabei entstehende Relaxation erforderte eine zweimalige Lastmessung, eine am Anfang und eine am Ende jeder Laststufe (Phasen I und III in Bild 18). Bei der Auswertung der Versuchsresultate wurde den gemessenen Verformungen stets die Endlast zugeordnet. Da die Spannungs-Dehnungs-Diagramme der StahlProbestäbe und der Betonprismen in ähnlicher Weise aufgenommen wurden, konnten diese Diagramme direkt für die Zuordnung Spannung-Dehnung übernommen werden. Die Dauer der Laststufen ist in den Tabellen 8 aufgeführt. Der Versuch wurde jeweils nach der zweiten Entlastung über Nacht unterbrochen.

Die im Balken wirksamen Schnittkräfte wurden aus den Lastmessungen am Pendelmano- 
meter der Zugpresse ermittelt. Die veränderlichen Schnittkräfte wurden jeweils auf die Mitte des Messbereiches ( $v g l$. Bild 19) bezogen. Die wesentlichen Verformungsmessungen wurden an je zwei $60 \mathrm{~cm}$ langen Balkenabschnitten vargenommen. Der erste Abschnitt diente zur Messung der Stahldehnungen (Detail-Messbereich), im zweiten Abschnitt, dessen Rissverhalten durch keine Messfenster gestört war, wurden die Betondehnungen und das Rissbild (Detail-Rissbereich) aufgenommen. Die Anordnung der Messstellen ist in Bild 19 wiedergegeben. Die folgenden Verformungsmessungen wurden durchgeführt.

a) globale Verformungen

- Verdrehungen in 7 Messquerschnitten mit einem induktiven Neigungsmesser (Anzeige in 1/100 Grad).

- Durchbiegungen in 7 Messquerschnitten mit auf den Balken gestellten Massstäben (Flexometer) durch Nivellieren (Ablesung auf $1 / 10 \mathrm{~mm}$ ).

- Horizontale Ausbiegungen in 7 Messquerschnitten mit an den Balken gehaltenem Masstab und Theodolit (Ablesung auf $1 / 10 \mathrm{~mm}$ ).

b) lokale Verformungen im Detail-Messbereich

- Längseisendehnungen mit induktiven Setzdehnungsmessern, Basis $60 \mathrm{~cm}$ (Anzeige in $2 / 1000 \mathrm{~mm}$ ) und $20 \mathrm{~cm}$ (Anzeige in $1 / 1000 \mathrm{~mm}$ ).

- Bügeldehnungen mit induktiven Setzdehnungsmessern, Basis $20 \mathrm{~cm}$ und $10 \mathrm{~cm}$ (Anzeige in $1 / 1000 \mathrm{~mm}$ ).

- Schiebungen und Krümmungen durch Ausmessen der Abschnittsseiten und diagonalen mit induktiven Setzdehnungsmessern, Basis $74 \mathrm{~cm}, 60 \mathrm{~cm}$ und $43 \mathrm{~cm}$ (Anzeige in $2 / 1000 \mathrm{~mm}$ ).

c) lokale Verformungen im Detail-Rissbereich

- Betondehnungen mit induktivem Setzdehnungsmesser, Basis $10 \mathrm{~cm}$.

- Rissbreiten auf Höhe der Zugarmierung und in Seitenmitte der vorderen und hinteren Querschnittscheibe mit einem Rissemikroskop (Ablesung auf $1 / 100 \mathrm{~mm}$ ).

Die induktiven Setzdehnungsmesser wurden auf Messbolzen aufgesetzt, die mit einem Schnellklebestoff auf der freiliegenden Stahl- resp. Betonoberfläche aufgeklebt waren. Mit diesem Verfahren wurde ein einwandfreies Ansetzen der Messinstrumente bis zum Bruch gewährleistet. 


\subsection{Trag- und Bruchverhalten}

\subsubsection{Bruchlasten}

Die in den Balkenversuchen erreichten Bruchlasten werden in Tabelle 7 und in Bild 20 mit den nach Abschnitt 2.3.2 berechneten Werten verglichen. Tabelle 7 enthält ausserdem die beobachteten Fliesslasten und die nach Gleichung (14a) bzw. (15a) bestimmten Diagonalenneigungswinkel der massgebenden Querschnittscheiben.

Auffallend ist bei dieser Gegenüberstellung die durchschnittlich um $44 \%$ höher liegende experimentelle Bruchlast gegenüber der am Scheibenmodell berechneten Traglast. Ungefähr $5 \%$ sind dabei auf die nicht idealen Lagerbedingungen (Schiefstellung der Lager + Lagerreibung) in den Auflagerrahmen der Versuchsanlage zurückzuführen. Der verbleibende Rest von etwa $40 \%$ kann nur durch die am Scheibenmodell nicht in Rechnung gestelite Plattenwirkung bedingt sein.

Die Vermutung, dass der Unterschied zwischen theoretischer und experimenteller Bruchlast fast ausschliesslich auf die Plattenwirkung der Querschnittscheiben zurückzuführen ist, wird durch die Versuche vollauf bestätigt. Dazu braucht man lediglich die in den Auflagerebenen gemessenen Haltekräfte der hinteren Platte in Bild 21 zu betrachten. Durch die Verdrehung des Lastarmes bzw. des Querträgers wird die hintere Platte nach aussen gedrückt. Einer solchen Bewegung würde praktisch kein widerstand entgegengebracht, wäre diese Platte in den Auflagerebenen nicht gehalten. Die entsprechenden Haltekräfte ergeben demnach ein aussagekräftiges Bild der Plattenwirkung. In Bild 21 sind die Haltekräfte der Laststufen 10 bis 18 für den Versuchsbalken TW, aufgetragen. Sie zeigen einen annähernd linearen Verlauf mit der Lastarmverdrehung, d.h. sie sind ungefähr proportional zur Plattenverformung. Die Grösse der Haltekräfte entspricht ohne weiteres den aufgetretenen Deformationen, wenn man an die relativ grosse Plattensteifigkeit denkt.

Die geringsten Anteile für die Plattenwirkung entstehen dort, wo die Plattensteifigkeit durch grosse Risse herabgesetzt ist. Die Hauptanteile liefern jene Querschnittsbereiche, welche durch die Wirkung einer Längsdruckkraft eine grössere Biegesteifigkeit aufweisen. Diese Bereiche sind in der Balken-Skizze des Bildes 21 als schraffierte Flächen dargestellt. Demzufolge waren beim Balken TW, vor allem die obere und die hintere Platte verantwortlich für eine über die Scheibenwirkung hinausgehende Erhähung der Traglast. Nimmt man an, dass die Plattenwirkung der oberen Platte dieselbe Grössenordnung wie in der hinteren Platte aufwies, so kommt man mit Hilfe der gemessenen Haltekräfte tatsächlich auf die experimentellen Bruchlasten.

In diesem Zusammenhang sei bemerkt, dass es im Bruchzustand im Gegensatz zum ungerissenen Zustand wenig sinnvoll ist, bei der Plattenwirkung zwischen einem Verwindungsanteil (St. Venant'scher Torsionswiderstand) und einem Biegeanteil zu unterscheiden. Grund dafür sind die sehr komplexen Steifigkeitsverhältnisse (vgl. Bild 21), welche stark von der Grösse und dem Verlauf der Risse abhängig sind. So konnte z.B. an allen für das Versagen massgebenden Querschnittscheiben beobachtet werden, wie sich die Rissneigungen auf der Innenseite der Versuchsbalken nach und nach dem Riss- 
verlauf auf der Aussenseite anglichen. Von St. Venant'scher Torsion zu sprechen, wie man sie von der Elastizitätstheorie her kennt, dürfte demnach fehl am Platze sein.

Ein weiterer Beweis für die vorliegende Erklärung wird durch den deutlich unter der Bruchlast liegenden Fliessbeginn erbracht. Bei allen Versuchsbalken kam zuerst der massgebende Gurtstab an der Stossstelle der Längsarmierung (siehe Bild 2) ins Fliessen, was an einer Messstrecke von $20 \mathrm{~cm}$ beobachtet werden konnte. Da die Plattenwirkung deformationsbedingt ist, wird sie erst nach Fliessbeginn richtig ins Spiel kommen. Die in Tabelle 7 und Bild 20 eingetragenen experimentellen fliesslasten zeigen dies deutlich, denn die Last konnte nach Fliessbeginn noch beträchtlich gesteigert werden, obwohl in der für den Bruch massgebenden Querschnittscheibe keine wesentliche Kräfteumlagerung mehr stattfand.

Die Plattenwirkung ist in erster Linie von der gewählten Querschnittsdicke $t$ abhängig. Bei dünnwandigen Querschnitten spielt die Plattenwirkung praktisch keine Rolle. Sie gewinnt an Bedeutung, je dickwandiger ein Querschnitt ausgeführt ist. Es muss hier betont werden, dass die Versuchsbalken $T W_{1}$ bis $T W_{5}$ dermassen gedrungene Querschnitte aufwiesen, wie sie in der Praxis kaum zu finden sind. Folglich fällt bei praktisch vorkommenden Tragwerken die Plattenwirkung weit weniger ins Gewicht. Die am Scheibenmodell berechneten Bruchlasten stimmen dann besser mit den tatsächlichen Werten überein.

Für die Interpretation der gemessenen Bruchlasten ist neben der in Rechnung gestellten Scheibenwirkung die Plattenwirkung nicht zu vergessen. Die Bedeutung der Plattenwirkung in den vorliegenden Balkenversuchen geht aus Bild 22 hervor. Bild 23 zeigt die für die Scheibenwirkung typische Querschnittsverwölbung.

\subsubsection{Tragverhalten}

Nachfolgend wird das Tragverhalten bis zum Bruch der einzelnen Versuchsbalken beschrieben. Die Tabellen 8 enthalten die Anfangs- und Endlasten sowie die zeitliche Dauer jeder einzelnen Laststufe. Einen Ueberblick über das Tragverhalten vermitteln auch die Last-Verformungs-Kurven der Bilder 47 bis 50.

\section{Balken $\underline{T W}_{1}$}

Im ungerissenen Zustand verformte sich der Versuchsbalken entsprechend seiner Steifigkeit nur wenig. Nach dem Auftreten der ersten Risse, die sich in jeder Balkenhälfte schraubenförmig um den Versuchskörper wanden, nahm die Steifigkeit sukzessive ab. Die Risse auf der Balkeninnenseite waren anfänglich stark gegenüber den Rissen auf der Aussenseite geneigt, was den grossen St. Venant'schen Torsionsanteil beim Entstehen der Risse bestätigt. Die weitere Entwicklung entsprach einem auf Biegung und Schub beanspruchten Träger, wenn man von der Verwindung der Querschnittscheiben absieht.

Die Armierung der unteren Gurtstäbe war wesentlich kleiner als die der oberen Gurtstäbe. Erwartungsgemäss kamen daher die unteren Längseisen auf der Balkenvorderseite (Gurtstab 7) ins Fliessen. Die Bügelarmierung wurde für den Versuchsbalken TW, so gewählt, dass sich in der massgebenden vorderen Querschnittscheibe ein Fachwerk mit etwa unter $45^{\circ}$-geneigten Betondiagonalen ausbildete. Die Bügeldehnungen überschritten in allen Bügelmessstellen der Balkenvorderseite ihre Fliessdehnung. 
Mit dem Fliessen des Gurtstabes 7 und der in der Querschnittscheibe 6 liegenden Bügel hätte man bereits die Traglast erreicht, wenn nur die Scheibenwirkung eine Rolle gespielt hätte. Die Last konnte jedoch dank der Plattenwirkung noch beträchtlich gesteigert werden, allerdings nahmen in dieser Phase die Deformationen sehr stark zu. Schliesslich zeigten sich deutliche Spuren grosser Druckbeanspruchungen, vor allem in den Diagonalen der Balkenvorderseite (siehe Bild 24). Nach der Laststufe 18 (vgl. Bild 47) konnten die Deformationen ohne nennenswerte Steigerung der Belastung erhöht werden, bis der Versuch infolge eines lokalen Versagens bei einem Auflager abgebrochen werden musste.

EaIken_I $\mathbf{W}_{2}$

Im Unterschied zum Versuchsbalken TW 1 waren hier die oberen Gurtstäbe schwächer ausgebildet. Es musste sich aus diesem Grunde ein vällig anderer Bruchmechanismus ausbilden. Dies zeigt Bild 26. Während sich bei allen anderen Versuchsbalken die vordere Querschnittscheibe nach unten bewegte, wurde beim Balken $T W_{2}$ die hintere Querschnittscheibe nach oben gedrückt. Dieser Mechanismus wurde durch das Fliessen des Gurtstabes 3 und der in der Scheibe 2 liegenden Bügel bewirkt. Ausserdem kamen die Bügel der Balkenvorderseite ins Fliessen, ohne dass dort ein Mechanismus beobachtet werden konnte.

Nach dem Ueberschreiten der Maximallast erfolgte der Bruch schlagartig durch Abplatzen der äusseren Betonschale auf der Vorderseite (siehe Bild 26) und durch eine Zerstörung der Druckzone auf der Innenseite des Balkens hinten unten. Dieses Versagen war bedingt durch die sehr grossen Plattenverformungen, warüber noch in Abschnitt 4.3 berichtet wird.

\section{BaIken $I W_{3}$}

Der Versuchsträger $T_{3}$ war mit dem Balken $T W_{1}$ identisch. Er wurde jedoch einer anderen Beanspruchung unterworfen, indem die Lastexzentrizität um die Hälfte reduziert wurde.

Trotz der unterschiedlichen Belastungsanordnung war die massgebende Querschnittscheibe 2 gleich beansprucht wie bei $T W_{1}$. Aus diesem Grunde zeigte der Balken $T_{3}$ mit Ausnahme unwesentlicher Unterschiede dasselbe Trag- und Bruchverhalten wie der Versuchsbalken $T_{1}$. Das Versagen erfolgte durch eine an der Aussenseite beginnende Zerstörung der Betondiagonalen, wie sie in Bild 25 zu sehen ist.

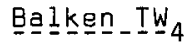

Gegenüber dem Versuchsbalken $T_{3}$ zeichnete sich der Balken $T_{4}$ durch eine um etwa die Hälfte reduzierte Bügelarmierung aus. Wird als Bruchmodell ein räumliches Fachwerk mit variabler Diagonalenneigung angenommen, so müssen trotz der einseitigen Reduktion der Bügelarmierung auch die massgebenden Längseisen (Gurtstab 7) ins Fliessen kommen, was im Versuch eindeutig festgestellt wurde. Die dadurch bedingte Flacherstellung der Druckdiagonalen konnte anhand der neu auftretenden Risse beobachtet werden. Allerdings wich das Rissbild im Durchschnitt nicht stark van der $45^{\circ}-$ Neigung ab (vgl. Bild 27), da es durch die starke Plattenverwindung beeinflusst wurde.

Nach dem Fliessen des Gurtstabes 7 und der vorderen Bügel erreichten auch die Bügel 
auf der Balkenhinterseite ihre Fliessspannung, was erwartungsgemäss keine Aenderung des Bruchmechanismus bewirkte. Wie bei den Versuchsbalken $T_{1}$ und $T_{3}$ stellte sich ein Mechanismus ein, bei dem sich die vordere Querschnittscheibe nach unten bewegte. Der Bruch erfolgte durch eine Zerstörung der Betondiagonalen auf der Balkenvorderseite.

\section{Balken_I $\underline{W}_{5}$}

Der Versuchsbalken $T_{5}$ entsprach dem Balken $T_{4}$ mit Ausnahme der Vorspannung der unteren Gurtstäbe. Um die Träger miteinander vergleichen zu können, wurden ungefähr gleich grosse Fliesskräfte der unteren Gurtstäbe gewählt. Dabei wurden die verwendeten Litzen derart vorgespannt, dass sie die Streckgrenze etwa bei der gleichen zusätzlichen Dehnung erreichten wie die schlaffe Armierung. Dementsprechend wiesen die Balken $T W_{4}$ und $T W_{5}$ mit Ausnahme der Risslast ein analoges Tragverhalten auf (Bild 50 ). Der Unterschied in der Risslast war nicht so ausgeprägt, wie man dies aufgrund von Vergleichen an Biegebalken erwarten könnte, weil der im Zugbereich liegende Gurtstab 3 (oben) nicht vorgespannt war.

Der Balken $T_{5}$ ging genau gleich wie der Balken $T_{4}$ zu Bruch. Dies verdeutlicht Bild 28. Der Bruch hätte bei hundertprozentigem Verbund schon bei kleineren Verformungen durch ein Reissen der Spannarmierung im Gurtstab 7 erfolgen können, was aber nicht eintrat, weil der Verbund zwischen Stahl und Beton gegen den Bruch hin nicht mehr vollständig war. Die Dehnung der Vorspannlitze konnte sich so auf eine grössere Länge verteilen und der Bruch erfolgte nicht durch ein Stahlversagen sondern durch ein Versagen des Betons wie im Falle des Balkens $\mathrm{TW}_{4}$.

\subsubsection{Brucharten - Biegeschubbruch}

Im vorhergehenden Abschnitt über das Tragverhalten konnte gezeigt werden, dass bei allen untersuchten Balken die gleichen Erscheinungen dem Bruch vorausgingen und diesen schliesslich herbeiführten. Die beobachtete Bruchart kann gemäss der im Anhang dieses Berichtes auf Seite $A B$ angegebenen Klassifikation der Brucharten als Biegeschubbruch bezeichnet werden. Diese Einteilung scheint berechtigt, da in den Balkenversuchen eine Beeinflussung der Mechanismen durch die Plattenwirkung nicht beobachtet werden konnte. Ausserdem hat die Plattenwirkung bei Balken mit dünnwandigen Querschnitten eine viel geringere Auswirkung als bei den gedrungenen Versuchsbalken $T W_{1}$ bis $T W_{5}$. Bei Balken mit dünnwandigen Querschnitten wird demnach die Art des Versagens noch deutlicher als Biegeschubbruch zu erkennen sein.

Die Versuche zeigten, dass alle Balken unterarmiert waren, d.h. die massgebende Armierung kam vor dem Versagen des Betons ins Fliessen. Das damit verbundene duktile Bruchverhalten ermäglichte die Anwendung der Plastizitätstheorie zur Bestimmung der theoretischen Traglast.

\subsection{Beanspruchung der Armierung}

Die Dehnungen der Stahleinlagen wurden mit Setzdehnungsmessern der Basis 60, 20 und $10 \mathrm{~cm}$ gemessen ( $\mathrm{vgl}$. Kapitel 3). Die Genauigkeit der einzelnen Messungen betrug für die mittlere Basislänge etwa 0.05 Promille. Die Anordnung der Messstellen ist aus 
Bild 19 ersichtlich. In den Bildern 29 bis'33 sind für jeden Versuchsbalken die Mittelwerte der Stahlspannungen über den $60 \mathrm{~cm}$ langen Detail-Messbereich (vgl. Bild 19) aufgetragen. Da die Gurtstäbe im Prüfbereich gestossen sind, wurden die Spannungen der Zug-Gurtstäbe 3 und 7 (hinten oben resp. vorne unten) auf den jeweils kleineren Armierungsquerschnitt bezogen. Ausserdem wurden für die Längseisen die theoretischen Stahlspennungen bei Laststufe 1 (Grundlast) mitberücksichtigt. Die Berechnung dieser Ausgangswerte erfolgte nach den Beziehungen der Elastizitätstheorie aus den bei Laststufe 1 vorhandenen Schnittkräften. Für die Mittelwerte der Bügelspannungen wurden pro Balken fünf Bügel auf der Balkenvorder- und Hinterseite sowie drei Bügel in der oberen Scheibe ausgemessen.

Zum Vergleich mit den gemessenen Kurven sind in den Bildern 29 bis 33 für die massgebenden Bügel und Längseisen auch die am Fachwerkmodell bestimmten theoretischen Werte eingetragen. Diese Geraden ergeben sich aus den Gleichungen (13), wenn man für die Diagonalenneigungen die im Bruchzustand gültigen Werte von Tabelle 7 einsetzt und die so erhaltenen Bügel- und Gurtstab-Kräfte durch die entsprechenden Stahlquerschnit"te dividiert. Die Berechnung der Stahlspannungen im gerissenen Zustand des vorgespannten Balkens $\mathrm{TW}_{5}$ erfolgt nach den Methoden teilweise vorgespannter Bauteile. Durch eine in Spanngliedhähe eingeführte fiktive Kraft wird der Beton zuerst spannungsfrei gemacht. Die dieser fiktiven kraft entgegengesetzte kraft wirkt dann zusammen mit den Schnittkräften (16) auf das räumliche Fachwerk. Der Unterschied zwischen Theorie und Messung ist in den Bildern 29 bis 33 nicht zu übersehen. Er beruht, wie dies in Abschnitt 4.1 .1 gezeigt wurde, in erster Linie auf der vernachlässigten Plattenwirkung.

Bei der Interpretation der Messungen ist die Anordnung und Lage der Messstellen (vgl. Bild 19) zu beachten. Die Messung erfolgte immer von der Aussenseite her. Dabei wurden die induktiven Setzdehnungsmesser auf Messbolzen aufgesetzt, die bei den Bügelmessstellen etwa $1 \mathrm{~cm}$ und bei den Längseisenmessstellen etwa $2 \mathrm{~cm}$ vom Aussenrand des Querschnitts entfernt waren. Die exzentrische Lage der Messstellen in den Querschnittscheiben wirkte sich für die Bügelmessungen praktisch nicht aus, da die Querschnittsform in den Versuchen mehr oder weniger erhalten werden konnte. Die Längseisendehnungen hingegen wurden durch die exzentrische Lage der Messstellen spürbar beeinflusst, da je nach Lage ein grösserer oder kleinerer Anteil der Plattenverformung mitgemessen wurde. Dieser Anteil äussert sich vor allem bei den Gurtstäben 1 und 5 (hinten unten bzw. vorne oben), die zusammen mit dem umliegenden Beton eine Druckzone bilden müssen. Die in den Bildern 29 bis 33 dargestellten Messwerte weisen auf einen starken Dehnungs - resp. Spannungsgradienten über die Querschnittsdicke hin und unterstreichen die Tatsache, dass der Unterschied zwischen Versuch und Theorie in der vernachlässigten Plattenwirkung liegt.

Die Bügelspannungen auf der Oberseite sind durch die Verankerung der seitlichen Bügelabschnitte zu erklären. Die Oberseite des Balkens $T_{2}$ war durch das Fliessen des Gurtstabes 3 stärker aufgerissen wie bei den übrigen Balken. Folglich waren die seitlichen Bügelkräfte im Beton der oberen Scheibe etwas schlechter verankert und die oberen Bügel etwas stärker beansprucht. Diese Verankerung begründet zusammen mit der aus der Verwindung entstandenen Rissbildung die gemessenen Bügelspannungen auf der Balkenoberseite.

Abgesehen von der Plattenwirkung und der damit zusammenhängenden Unterschiede im Spannungsverlauf entwickelten sich die Stahlspannungen gemäss den am Fachwerkmodell 
errechneten Werten. Insbesondere konnte bei allen Versuchsbalken das theoretisch erwartete Fliessen der massgebenden Bügel und Gurtstäbe beobachtet werden. Bei den Versuchsbalken $T W_{1}, T W_{3}, T W_{4}$ und $T W_{5}$ entwickelten sich dieselben Bruchmechanismen. Aus diesem Grunde verliefen die Stahlspannungen dieser Balken ziemlich ähnlich. Infolge des späteren Rissbeginns beim vorgespannten Balken $T_{5}$ war der Spannungszuwachs im gerissenen Zustand ausgeprägter. Der Spannungsverlauf des Balkens $\mathrm{TW}_{2}$ unterschied sich in Uebereinstimmung mit dem eingetretenen Bruchmechanismus deutlich von allen übrigen Balken.

\subsection{Beanspruchung des Betons}

Die Stauchungen der Betonoberfläche wurden mit Setzdehnungsmessern der Basis $10 \mathrm{~cm}$ an den in Bild 19 angegebenen Messstellen ermittelt. Die Genauigkeit der einzelnen Messung betrug etwa 0,1 Promille. Aufgrund der grossen Torsions- bzw. Schubbeanspruchung entwickelten sich bei allen Balken Diagonalrisse, durch die die Messungen der zur Balkenaxe parallelen Messstellen gestört wurden. Dargestellt sind in Bild 34 die Stauchungen der Betanoberfläche auf der für das Versagen massgebenden Querschnittscheibe unter einer Neigung von $45^{\circ}$ gegenüber der Balkenaxe. In Bild $34(a)$ sind die Betonstauchungen in Funktion der Belastung aufgetragen. Interessant war das starke Anwachsen der Stauchungen nach dem Fliessbeginn der Armierung obwohl infolge Gleichgewicht im Fachwerk eine Vergrösserung der Diagonaldruckkraft nicht mehr möglich war. Dieser Zuwachs musste also durch die Verformung selbst verursacht werden. Es handelte sich dabei um den in [2] beschriebenen Verwindungseffekt. Die grössten Randstauchungen aus dieser Verwindung treten unter $45^{\circ}$ auf und haben nach [2] den Betrag :

$$
\varepsilon_{b}(\vartheta)=-\frac{d v}{d x} \cdot \frac{t}{2}
$$

Der Verwindungsanteil ist demnach eine lineare Funktion der gemessenen spezifischen Verdrehung $v_{60}$.

$$
\varepsilon_{b}(\vartheta)=-\frac{\vartheta_{60}}{60} \cdot \frac{8}{2}=-\frac{\vartheta_{60}}{15}
$$

Den linearen Zusammenhang zwischen Betonstauchung und spezifischer Verdrehung veranschaulicht Bild $34(b)$. Erst bei grosser Rissöffnung traten die Diagonalen etwas aus der verwundenen Fläche heraus und die Dehnungszunahme verflachte sich. Die den Randstauchung entsprechende, unter $45^{\circ}$ zur Balkenaxe geneigte Druckspannung setzt sich aus einem Strebenkraft- und einem Verwindungsanteil zusammen. Dies zeigt die aus [2] übernommene Gleichung:

$$
\sigma_{b}=-\left(2 \tau+E_{b} \cdot \frac{\vartheta_{60}}{15}\right)
$$

Die nominellen Schubspannungen $\tau$ können mit Hilfe der Beziehungen (13), in welchen die Scheibenquerkräfte durch die entsprechenden Schubflächen zu dividieren sind, bestimmt werden. Für die Scheibe $k$ erhält man:

$$
\tau_{k}=\frac{Q_{k}}{a_{k} \cdot t}
$$

Wie aus Bild $34(b)$ zu entnehmen ist, spielen die Betonstauchungen aus der Verwindung 
eine bedeutende Rolle für die Bruchart. Sie sind bei der Festlegung der oberen Schubspannungsgrenze unbedingt zu berücksichtigen, um ein vorzeitiges Versagen der Betondiagonalen zu verhindern. Für eine bestimmte Stahldennung (z.B. Fliessbeginn) wird die Verdrehung und damit auch die Verwindung umso kleiner, je weiter die Querschnittscheiben auseinanderliegen, d.h. je grösser die Aussenabmessungen eines Querschnittes sind. Nach Gleichung (17) vergrössert sich die aus der Verwindung stammende Randstauchung mit zunehmender Wandstärke $t$, so dass die Gedrungenheit eines Querschnittes, die durch das Verhältnis der Wandstärke zu den Aussenabmessungen ausgedrückt werden kann, eine Aussage über die zu erwartende Betonbeanspruchung gibt.

Neben dem vorzeitigen Versagen der Betondiagonalen ist auch der vorzeitige Sprödbereich von druckbeanspruchten Gurtstäben zu verhindern. Dabei sind wie bei der Festlegung der oberen Schubspannungsgrenze die durch die Plattenverformung bedingten Betonstauchungen zu berücksichtigen.

4.4 Verformungen

\subsubsection{Schiebungen und Krümmungen}

Die Schiebungen $\gamma$ und die Krümmungen $\frac{d \varphi}{d x}$ wurden im Detail-Messbereich (vgl. Bild 19) durch Ausmessen der Abschnittsseiten und -diagonalen mit Setzdehnungsmessern bestimmt. Die Berechnung der Winkeländerungen in den vier Ecken des rechteckigen Messabschnittes aus den gemessenen Grössen und die damit mögliche Bestimmung der Schiebungen und Krümmungen ist in [2] eingehend beschrieben.

Die auf diese Weise ermittelten Schiebungen und Krümmungen sind in den Bildern 35 bis 44 für alle Querschnittscheiben und Balken dargestellt. Auf den Zusammenhang zwischen Schiebungen und Dehnungen wird hier nicht näher eingegangen, da dies schon in den Berichten [2], [3], [4] und [5] eingehend untersucht wurde. Der lineare Zusammenhang zwischen Längsdehnungen und Krümmung ist evident. Als Mass für die Krümmung wird der gemessene Dehnungsunterschied $\frac{d \varphi}{d x} \cdot 43 \mathrm{~cm}$ der beiden in einer Scheibe liegenden Gurtstäbe (Messstellenabstand $=43 \mathrm{~cm}$ ) aufgetragen.

Die gemessenen Werte entsprechen durchaus den Erwartungen. Interessant ist das unterschiedliche Verformungsverhalten der Balken vor und nach Fliessbeginn der Armierung. Man sieht sehr schön, wie sich das Verformungsverhalten nach Fliessbeginn den in den Bildern 11 und 12 skizzierten Bruchmechanismen annäherte.

\subsubsection{Durchbiegungen}

Die Durchbiegungen der einzelnen Querschnittscheiben wurden aus den KlinometerFlexometer-Messungen unter Annahme der Querschnittserhaltung ermittelt. In Bild 45 sind die Durchbiegungen vor Fliessbeginn und in Bild 46 die unter Maximallast aufgetretenen Werte dargestellt. Beim Vergleich der beiden Bilder sind die eingetretenen Mechanismen deutlich zu erkennen. Erwartungsgemäss nahmen bei den Balken TW 1 , TW 3 , $\mathrm{TW}_{4}$ und $\mathrm{TW}_{5}$ die Durchbiegungen der Balkenvorderseite sehr stark zu, währenddem beim Balken TW 2 die Durchbiegungen der oberen und vor allem der hinteren Scheibe nach Fliessbeginn anwuchsen.

Neben den über die Balkenlänge aufgetragenen Durchbiegungen der Bilder 45 und 46 
sind die Durchbiegungen der massgebenden Querschnittscheiben in Balkenmitte auch in Funktion der Belastung angegeben. Dies zeigen die Bilder 47 bis 50, die vor allém das in Abschnitt 4.1 .2 beschriebene Tragverhalten der Versuchsbalken illustrieren.

\subsection{Rissverhalten.}

\subsubsection{Risslasten}

Bei allen Versuchsbalken traten vorerst Biegeschubrisse auf, die sich bei den Balken $\mathrm{TW}_{1}$ bis $\mathrm{TW}_{4}$ aus Anrissen auf der Balkenvorderseite unten und beim Balken $\mathrm{TW}_{5}$ aus Anrissen auf der Balkenhinterseite oben entwickelten. Die ersten Risse traten ungefähr im Abstand h/2 vom Mittelquerträger auf, weshalb dieser Querschnitt (Querschnitt B-B) zur Bestimmung der Vergleichsspannungen bei Rissbeginn herangezogen wurde.

Die bei Rissbeginn vorhandenen Lasten sind in Tabelle $g$ aufgeführt. Für den Vergleich mit der Biegezugfestigkeit des Betons genügen die Normalspannungen $\sigma_{R}$, da diese die Schubspannungen im Querschnitt B-B bei weitem übertrafen. Die Vergleichsspannungen $\sigma_{R}$ wurden nach Gleichung (7) berechnet. Sie entsprechen den Mittelwerten der Normalspannungen über die Querschnittsdicke $t$ und dürften für den Rissbeginn im Querschnitt B-B massgebend sein. Die SIA-Norm 162 (1968) nimmt für die an Betonprismen ermittelte Biegezugfestigkeit den Wert von $(2,5 \div 3,0) \cdot \sqrt{B_{W}}$ an, was gemäss Tabelle 3 auch für den verwendeten Beton zutrifft. Diese Biegezugfestigkeit ist nach den Versuchsbeobachtungen massgebend für den Rissbeginn im Querschnitt B-B, da dort die Verhältnisse Schubzu Normalspannungen sehr klein sind. Bei stärkeren Schubspannungsanteilen würde der Beton schon früher reissen. Der berechnete, niedrigere Wert beim Balken TW $_{5}$ weist in diese Richtung, allerdings dürfte dafür auch die schlechtere Betonqualität auf der Balkenoberseite verantwortlich gewesen sein.

\subsubsection{Rissverlauf}

Die ersten Risse öffneten sich bei allen Balken senkrecht zur Hauptzugrichtung und verliefen spiralförmig um jede der beiden Balkenhälften. Allgemein wurde der Rissverlauf der in diesem Bericht beschriebenen Versuchsbalken sowohl durch die Scheibenals auch die Plattenwirkung bestimmt. Die Risse entwickelten sich gemäss der Scheibenwirkung (Neigung der Druckdiagonalen) und der Plattenwirkung (Verwindung). Letztere erreichte bei den untersuchten Balken mit offenen Querschnitten sehr grosse Werte (vgl. Bild 34) und beeinflusste den Rissverlauf an der Betonoberfläche entsprechend der grossen Wandstärke von $8 \mathrm{~cm}$ beträchtlich. Aus diesem Grunde wichen die Rissbilder auf der Vorder-Hinter- und oberseite der Balken im Mittel nicht stark von der $45^{\circ}$-Neigung ab. Bild 51 zeigt stellvertretend für alle Balken den Rissverlauf des Balkens $\mathrm{TW}_{2}$.

\subsubsection{Rissbreiten}

In einem $60 \mathrm{~cm}$ langen Detail-Rissbereich ( $\mathrm{ggl}$. Bild 19) wurden die Rissbreiten auf je zwei Linien der Vorder- und Hinterseite mit einem Risse-Mikroskop ausgemessen. Die Ablesegenauigkit betrug $1 / 100 \mathrm{~mm}$. Die ermittelten werte wurden auf Folien notiert, welche direkt neben den Messpunkten am Balken fixiert und photographiert wurden. Das Verfahren ist aus Bild 51 ersichtlich. 
Die mittleren Rissbreiten der grössten fünf Risse auf Höhe der massgebenden Längszugarmierung wurden in Bild $52(a)$ der Belastung gegenübergestellt. Die mittlere Rissbreite des Balkens $T_{4}$ wich von denjenigen der übrigen Balken ab, da durch den abrupten Uebergang der Längsarmierung lokal sehr hohe Dehnungen entstanden. Die maximalen Rissbreiten entlang den gleichen Messaxen sind in Bild 52(b) wiedergegeben.

Zusätzlich wurde in diesen Bildern die nach den CEB-Richtlinien als zulässig bezeichnete maximale Rissbreite im Gebrauchszustand von $0,2 \mathrm{~mm}$ eingetragen. Wie aus Bild 52 (b) hervorgeht, wurde diese Grenze bei der durch 1,8 dividierten experimentellen Bruchlast $P$ UE (schraffierter Bereich) zum Teil ganz wesentlich überschritten. Die grossen Rissbreiten sind eindeutig auf die starke Plattenverformung zurückzuführen, die wegen des grossen Dehnungsgradienten über die Wandstärke grosse Rissöffnungen auf der Balkenoberfläche erzeugte.

Berücksichtigt man für die Bemessung hingegen nur die Scheibenwirkung wie beim theoretisch betrachteten Scheibenmodell, so erhält man im Gebrauchszustand bei der durch 1,8 dividierten theoretischen Bruchlast $\mathrm{P}_{U}^{\mathrm{Th}}$ ein vernünftiges Verformungs- und Rissverhalten (siehe Bild $52(b)$ ). 
Im Rahmen eines Forschungsprogrammes über das Verhalten von Stahlbetonbalken unter Torsion, Biegung und Querkraft wurden vom Institut für Baustatik, Abt. Massivbau der Eidgenössischen Technischen Hochschule in Zürich (ETHZ) fünf Versuche an Stahlbetonbalken mit offenen Querschnitten durchgeführt. Diese ergänzen die bisher durchgeführten Versuche an Stahlbetonbalken mit geschlossenen und vollen Querschnitten, welche in [2] bis [5] ausführlich beschrieben sind. Die Hauptvariablen (Armierung, Beanspruchung und Vorspannung) sind in Bild 1 zusammengestellt. Ausser der aufgebrachten Last wurden Stahl- und Betondehnungen, Verdrehungen und Durchbiegungen, Rissbreiten sowie Schiebungen und Krümmungen gemessen.

Die Ergebnisse dieser Versuche lassen sich wie folgt zusammenfassen:

- Die Vergleichsspannungen bei Rissbeginn der sich entwickelnden Biegeschubrisse lagen im Bereich der für reine Biegung (Biegezugfestigkeit) ermittelten Werte.

- Im gerissenen Zustand entwickelte sich ein räumliches Fachwerk mit den Längseisen als Gurtungen, den Bügeln als Pfosten und den Beton-Druckdiagonalen. Die durch die Umlagerung der Kräfte zwischen Längseisen und Bügeln bedingte endgültige Neigung der Druckdiagonalen wurde durch anfängliche Risse nicht beeinflusst.

- Neben der Scheibenwirkung, die sich in der Ausbildung eines räumlichen Fachwerkes äusserte, gewann die Plattenwirkung mit zunehmender Verformung an Bedeutung. Sie erreichte wegen der relativ dickwandigen Ausführung der Versuchsbalken eine beachtliche Grösse.

- Die Plattenwirkung war verantwortlich für eine Erhöhung der Traglast gegenüber der am Fachwerkmodell bestimmten theoretischen Bruchlast. Die gemessenen Maximallasten überschritten die theoretisch ermittelten Bruchlasten um etwa $40 \%$, während die am Fachwerkmodell vorausgesagten Mechanismen in allen Fällen zutrafen.

- Infolge der grossen Plattenverformungen traten sehr hohe Betonbeanspruchungen auf. So wurden die Gurtstäbe und die Betondruckdiagonalen neben der zentrischen Normalkraft nach Fachwerktheorie auch durch eine Krümmung beansprucht. Demzufolge spielen die Betonstauchungen aus der Plattenverformung eine bedeutende Rolle für die Art des eintretenden Bruches.

- Alle Versuchsbalken waren unterarmiert, d.h. die massgebende Armierung kam vor dem Versagen des Betons ins Fliessen. Die beobachtete Bruchart wies alle Merkmale eines Biegeschubbruches auf.

- Die Versuche zeigten, dass man bei einer Bemessung auf die theoretische Bruchlast des Fachwerkmodelles, wo die Plattenwirkung vernachlässigt wird, ein vernünftiges Verformungs- und Rissverhalten im Gebrauchszustand erhält. Daher sollte für die praktische Bemessung die Plattenwirkung im Bruchsicherheitsnachweis nicht in Rechnung gestellt werden. 
Dans le cadre d'un programme de recherche sur le comportement des poutres en béton armé sousmises à la torsion, à la flexion et à l'effort tranchant, on a procédé, à l'institut de Statique, section béton, de l'Ecole Polytechnique Fédérale (EPF) de Zurich, a des essais sur cinq poutres à sections ouvertes. Ces essais complètent ceux exécutés jusqu' à présent sur des poutres à sections pleines et fermés, essais qui ont été décrits en détail dans [2], [3], [4] et [5]. Les paramètres variés (armature, bras de levier de la charge appliquée, précontraintel sont rassemblés dans le tableau 1. En plus de la charge appliquée on a mesuré les déformations de l'acier et du béton, les rotations, les flexions et la largeur des fissures, ainsi que les déformations de cisaillement et les courbures.

Les résultats des essais peuvent se résumer de la façon suivante:

- Les contraintes de traction nominelles au commencement de la fissuration à partir des fissures de flexion atteignaient la résistance à la traction du bétan obtenue pour la flexion pure.

- A l'état fissuré, la structure portante agissait comme un treillis spatial avec l'armature longitudinale comme membrures, les étriers comme montants et des bielles comprimés en béton. L'inclinaison définitive des diagonales comprimés conditionnée par la transposition des forces entre des fers longitudinaux et les etriers n'était pas influencée par les fissures initiales.

- En plus des efforts contenus dans chaque parois de la poutre (treillis spatial), l'effet de plaque devenait de plus en plus important avec l'augmentation des déformations, à cause de la grande épaisseur des parois.

- Grâce à l'effet de plaque (pas consideré dans les calculs théoriques) la charge de rupture se trouvait au-dessus de celle donnée par la théorie du treillis. Les efforts mesurés maximaux dépassaient d'environ $40 \%$ les valeurs théoriques, tandis que les mécanismes prédits se trouvaient justes dans tous le cas.

- La déformation des plaques produisait des grandes déformations du béton. En plus de la force axiale centrée selon la théorie du treillis, les membrures et les bielles comprimées etaient aussi sousmises à des contraintes dues à la courbure. Celles-ci jouent un rôle important pour le genre de la rupture.

- Toutes les poutres étaient sous-armées, de sorte que l'armature déterminante a atteint la limite d'écoulement avant l'écrasement du béton. Le genre de rupture observé montrait les indices d'une rupture typique pour une poutre sousmise à la flexion et à l'effort tranchant.

- Les essais ont montré qu'un dimensionnement basé sur la charge de rupture théorique, calculée en négligeant l'effet de plaque, livrait un état de déformation et de fissuration raisonable pour les charges réelles. C'est pourquois pour un dimensionnement pratique, il ne faut pas tenir compte de l'effet de plaque dans le contrôle à la ruine. 
Five reinforced concrete beams with open cross sections were tested at the Institute of Structural Engineering, Swiss Federal Institute of Technology (ETH), Zurich, as part of a research program to study the behavior of reinforced concrete beams under combined torsion, bending and shear. These tests complete the previous test series on reinforced concrete beams with plain and hollow cross sections described in detail in [2], [3], [4] and [5]. The main parameters (reinforcement, excentricity of applied load, prestressing) are given in Fig. 1. At each stage of the tests steel strains, concrete strains, angles of twist, deflections, crack widths, shear strains, curvatures and applied loads were recorded.

The results of these tests may be summarized as follows:

- The nominal tensile stresses at the onset of cracks due to bending came up to the tensile strength of concrete obtained for pure bending (tensile strength in flexure).

- After cracking all beams worked as space trusses with the longitudinal bars acting as stringers, the stirrups acting as vertical ties and the concrete diagonals acting as inclined struts. The final inclination of struts determined by the redistribution of forces between transverse and longitudinal reinforcement was not affected by initial cracks.

- Beside the in-plane forces acting in each wall of the beams (space truss forces), the plate bending action became more and more important with increasing deformations, it reached a relatively high value due to the thickness of the walls.

- The plate bending action, which was not considered in the theoretical study, led to higher values of ultimate loads than predicted by theory. The measured ultimate loads exceeded the theoretical values calculated with the space truss model by about $40 \%$, whereas the failure mechanisms were as predicted.

- In accordance to the large bending deformation of plates high concrete strains were measured. The stringers and the compression diagonals were subjected to on axial force according to truss theory and an additional curvature. The concrete strains due to this curvature cannot be neglected for describing failure modes (ductile, not ductile).

- All test beams were under-reinforced, so that the reinforcement yielded before the failure of concrete. The observed failure modes showed the characteristics of a failure under bending and shear.

- It can be stated from the test results, that under service loads a good behavior relative to cracks and deformations is obtained, when the design bases on the theoretical carrying capacity of the truss model without considering the plate bending action. Therefore this should be neglected in practical ultimate load design. 
Der vorliegende Bericht wurde im Rahmen des Forschungsprogrammes "Torsion, Biegung und Querkiaft von Stahlbetonbalken" des Institutes für Baustatik. Abt. Massivbau, Eidgenässische Technische Hochschule Zürich, ausgearbeitet. Für die grosszügige finanzielle Unterstützung dieses Programmes möchten die Verfasser der

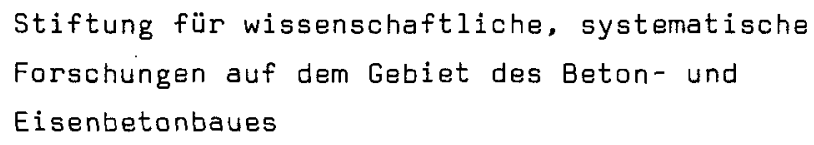

aufrichtig danken. Der experimentelle Teil der Untersuchungen wurde an der Eidgenässischen Materialprüfungs- und Versuchsanstalt (EMPA) in Dübendorf durchgeführt. Für dieses Entgegenkommen möchten die Verfasser Herrn Professor Dr. Th. Erismann, Direktionspräsident der EMPA sowie Herrn Dr. M. Ladner, Vorsteher der Abteilung Massivbau, ihren besten Dank aussprechen. Die Armierungsstähle für die Versuchsbalken wurden von den Firmen Monteforno Stahl- und Walzwerke AG, Bodio, Ferrowohlen Stahl- und Walzwerke $A G$, Wohlen, die Spannkabel von der Firma Losinger + Co. AG, Bern, in verdankenswerter Weise zur verfügung gestellt.

Bei der Versuchsdurchführung und der Gestaltung dieses Berichtes haben mitgearbeitet: Frl. S. Burki, die Herren K. Bucher, G. Göseli und L. Sieger. Herr Dipl. Ing. M. Baumann hat sich besonders bei den Messungen mit der neu entwickelten elektronischen Mess- und Datenerfassungsanlage eingesetzt. Für Ihre Mitarbeit sei den Genannten, wie auch den übrigen an der Versuchsdurchführung Beteiligten, bestens gedankt. 


\section{LITERATURVERZEICHNIS}

[1] Lampert P./Thürlimann B.: "Versuchsanlage für Balken unter Torsion-BiegungQuerkraft", Bericht Nr. 12, Juni 1967, Institut für Baustatik, ETH, Zürich (Sonderdruck Schweizer Archiv. Heft 9, 33. Jahrgang 1967).

[2] Lampert P./Thürlimann B.: "Torsionsversuche an Stahlbetonbalken", Bericht Nr. 6506-2, Juni 1968, Institut für Baustatik, ETH, Zürich.

13] Lampert P./Thürlimann B.: "Torsions-Biege-Versuche an Stahlbetonbalken", Bericht Nr. 6506-3, Januar 1969, Institut für Baustatik, ETH, Zürich.

[4] Lampert P./Lüchinger P./Thürlimann B.: "Torsionsversuche an Stahl- und Spannbetonbalken", Bericht Nr. 6506-4, Februar 1971, Institut für Baustatik, ETH, Zürich.

[5] Lüchinger P./Thürlimann B.: "Versuche an Stahlbetonbalken unter Torsion, Biegung und Querkraft", Bericht Nr. 6506-5, Juli 1973, Institut für Baustatik, ETH, Zürich.

[6] Lampert P./Wegmüller A./Thürlimann B.: "Einfluss der Dehngeschwindigkeit auf Festigkeitswerte von Armierungsstählen", Bericht Nr. 10, April 1967. Institut für Baustatik, ETH, Zürich (Sonderdruck Schweizerische Bauzeitung, 85. Jahrgang, Heft 14, 6. April 1967).

17] Wlassow W.S.: "Dünnwandige elastische Stäbe", Band 1, VEB Verlag für Bauwesen, Berlin, 1964.

[8] Kollbrunner C.F./Basler K.: "Torsion", Springer Verlag, Berlin/Heidelberg/ New York, 1966.

[9] Grob J.: "Traglast von Stäben mit dünnwandigen offenen Querschnitten". Institut für Baustatik, ETH, Zürich. Erscheint demnächst im BirkhäuserVerlag Basel und Stuttgart. 


\section{Längen, Flächen und Querschnittswerte}

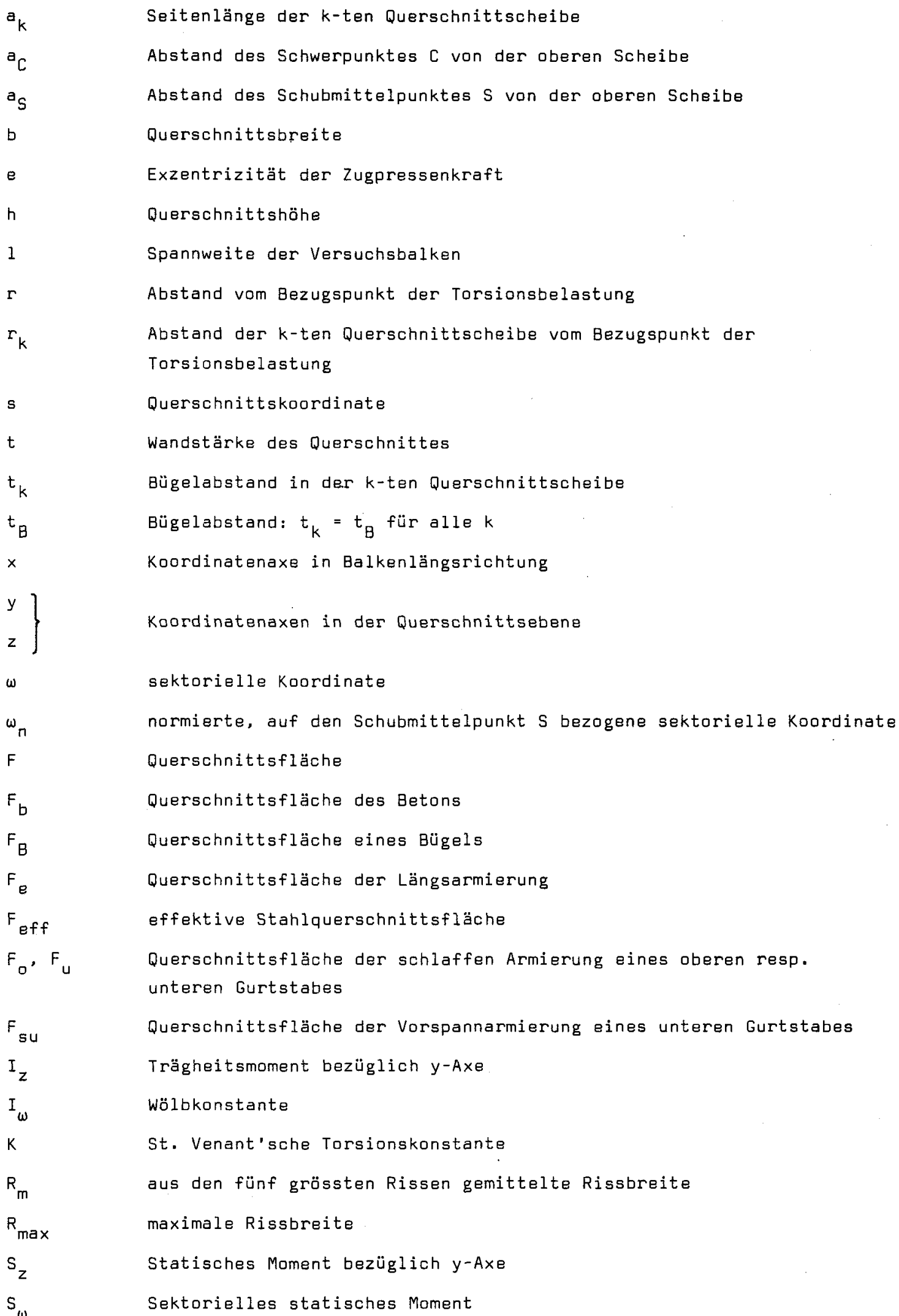




\section{Kraftgrössen}

\begin{tabular}{|c|c|}
\hline$m_{x}$ & Torsionsbelastung \\
\hline$P_{z}$ & Belastung in Richtung $z-A x e$ \\
\hline$B_{k}$ & Bügelzugkraft in der k-ten Querschnittscheibe \\
\hline$B_{f k}$ & Fliesskraft eines Bügels der k-ten Querschnittscheibe \\
\hline$B_{f}$ & Fliesskraft eines Bügels: $B_{f k}=B_{f}$ für alle $k$ \\
\hline $\mathrm{EI}_{z}$ & Biegesteifigkeit im ungerissenen Zustand bezüglich y-Axe \\
\hline $\mathrm{EI}_{\omega}$ & Wölbsteifigkeit im ungerissenen Zustand \\
\hline GK & Torsionssteifigkeit im ungerissenen Zustand \\
\hline$H_{1,2}$ & Haltekräfte der hinteren Platte in den Auflagerebenen \\
\hline$M_{y}$ & Biegemoment bezüglich $z$-Axe \\
\hline$M_{z}$ & Biegemoment bezüglich $y$-Axe \\
\hline$M_{\omega}$ & Wölbmoment \\
\hline $\mathrm{N}$ & Normalkraft \\
\hline$P$ & Zugpressenkraft \\
\hline$P_{A}$ & Zugpressenkraft am Anfang einer Laststufe \\
\hline$P_{E}$ & Zugpressenkraft am Ende einer Laststufe \\
\hline$P_{R}$ & Zugpressenkraft bei Rissbeginn \\
\hline$p_{f E}^{E x}$ & Experimentelle Fliesskraft \\
\hline$P_{U E}^{E X}$ & Experimentelle Maximallast \\
\hline$P_{u}^{T h}$ & Theoretische Maximallast \\
\hline$Q_{k}$ & Schubbeanspruchung der $k$-ten Querschnittscheibe \\
\hline $\begin{array}{l}Q_{y} \\
Q_{z}\end{array}$ & Querkräfte \\
\hline $\mathrm{T}$ & Torsionsmoment \\
\hline$T_{s}$ & St. Venant'sches Torsionsmoment \\
\hline$T_{w}$ & Wälbtorsionsmoment \\
\hline$z_{i}$ & Zugkraft des i-ten Gurtstabes \\
\hline$z_{f i}$ & Fliesskraft des $i$-ten Gurtstabes \\
\hline$Z_{k}$ & $\begin{array}{l}\text { aus der Schubbeanspruchung resultierende Längskraft in der k-ten } \\
\text { Querschnittscheibe }\end{array}$ \\
\hline
\end{tabular}

\section{Festigkeitswerte und Spannungen}

$\begin{array}{ll}\beta_{b z} & \text { Biegezugfestigkeit des Betons } \\ \beta_{p} & \text { Prismendruckfestigkeit des Betons } \\ \beta_{p d} & \text { dynamische Prismendruckfestigkeit des Betons } \\ \beta_{p s} & \text { statische Prismendruckfestigkeit des Betons } \\ \beta_{w} & \text { Würfeldruckfestigkeit des Betons }\end{array}$




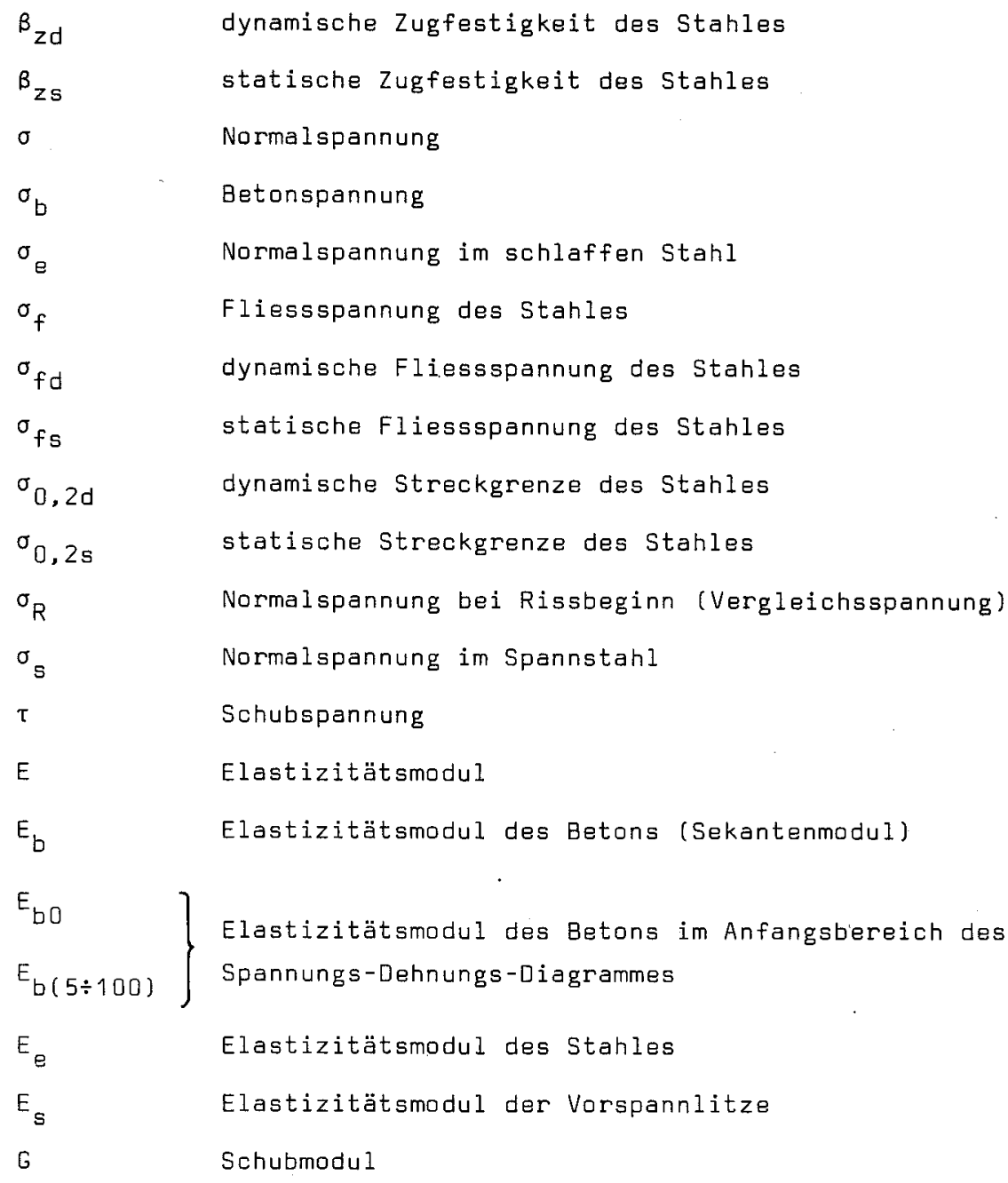

\section{Verformungen, Winkel und Zeitgrössen}

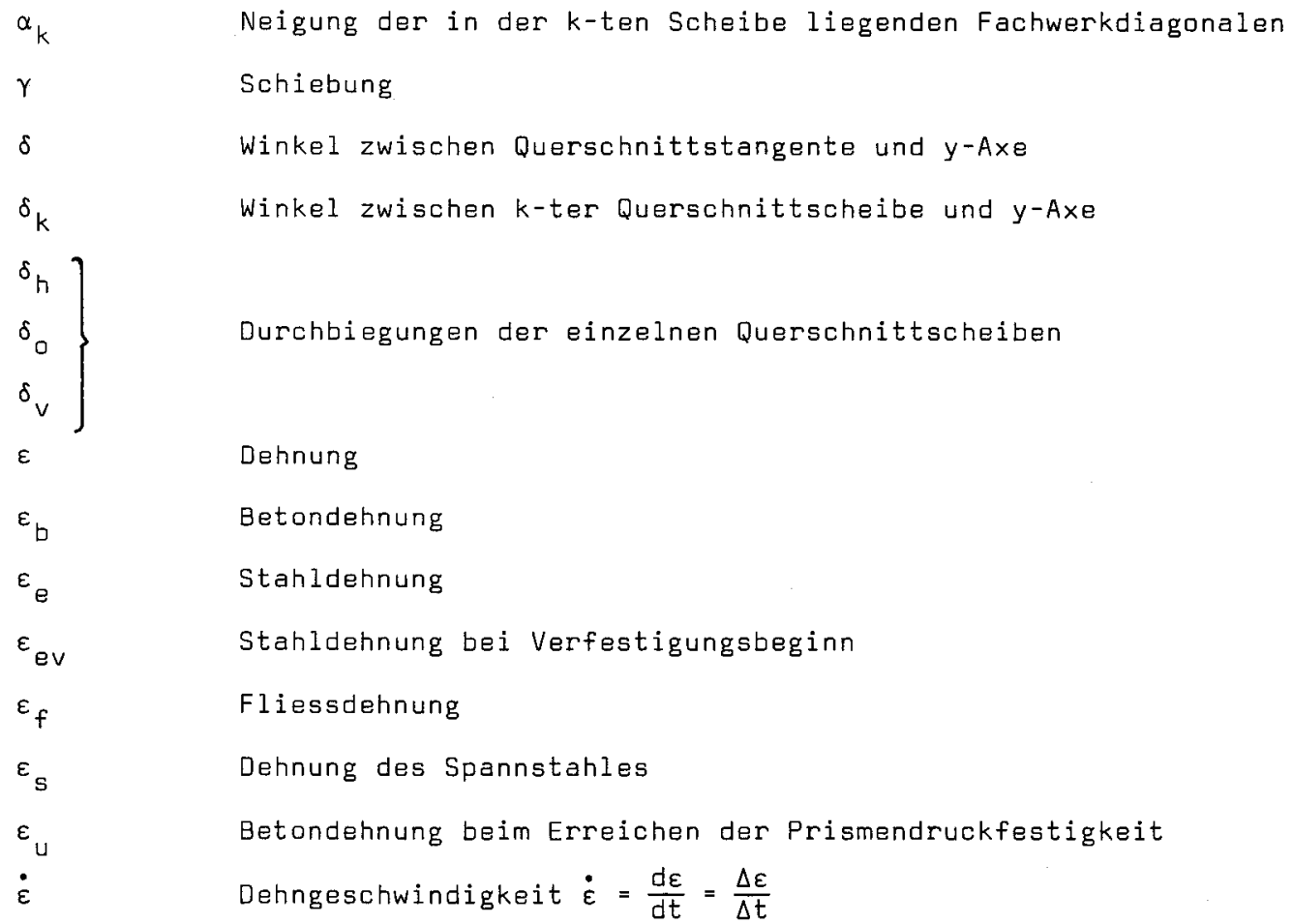




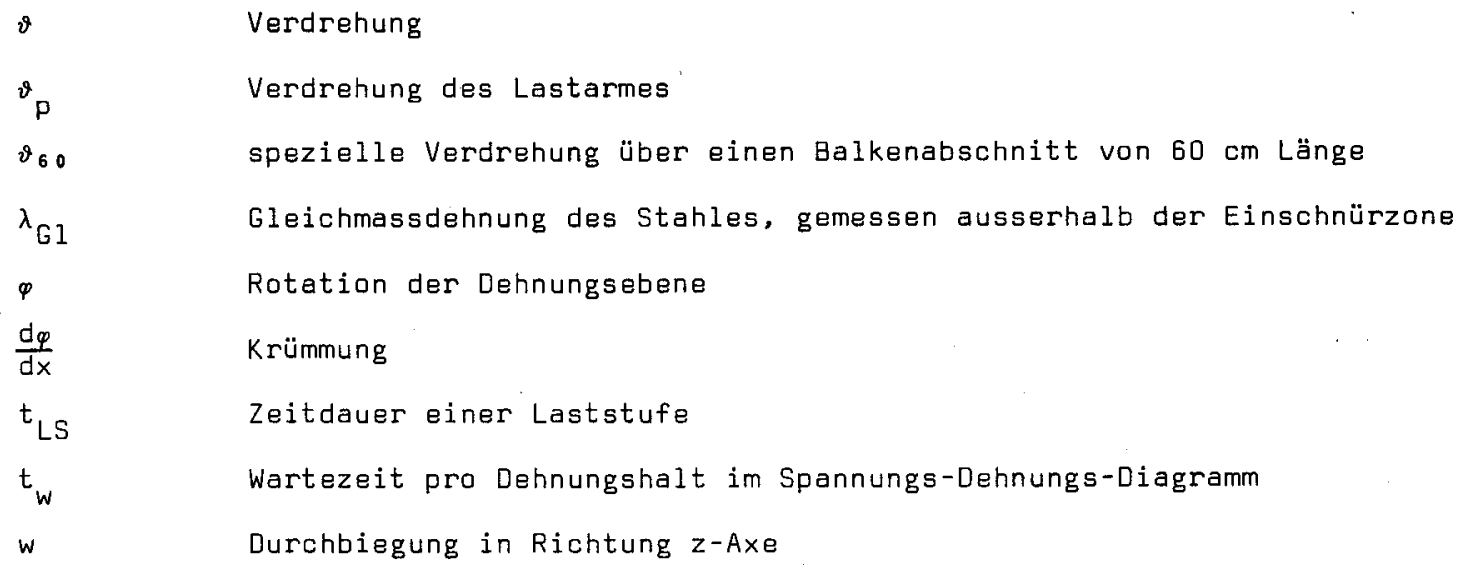

a

b

d

e

f

h auf Querschnittsrand bezogen

Beton

dynamisch

Stahl

Fliess-

hinten

auf $i-t e n$ Gurtstab bezogen

auf k-te Querschnittscheibe bezogen

auf Wandmittellinie bezogen; mittel

normiert

oben

Spannstahl; statisch

unten; ultimate

vorne

Anfang der Laststufe

Bügel

auf Schwerpunkt bezogen

Ende der Laststufe

Nu 11

Riss 
auf Schubmittelpunkt bezogen

(g) infolge Eigengewicht

(v) infolge Vorspannung 


\begin{tabular}{|c|c|c|c|c|c|c|c|c|c|c|}
\hline Nenn $\phi$ & Foff & $\begin{array}{c}\sigma_{t s} / \sigma_{0,2 s} \\
\dot{\varepsilon}=0\end{array}$ & $\begin{array}{l}\sigma_{f d} / \sigma_{0,2 d} \\
\dot{\varepsilon}=1,25 \% / \min \end{array}$ & $\begin{array}{l}\beta_{\mathbf{2 s}} \\
\dot{\varepsilon}=0\end{array}$ & $\begin{array}{c}\beta_{\mathbf{2 d}} \\
\dot{\varepsilon}=1,25 \% / \mathrm{min}\end{array}$ & $\varepsilon_{e v}$ & $\lambda_{G I}$ & $\psi$ & $E_{e} / E_{3}$ & $\begin{array}{l}\text { Anzahl } \\
\text { Proben }\end{array}$ \\
\hline $\mathrm{mm}$ & $\mathrm{cm}^{2}$ & $t / \mathrm{cm}^{2}$ & $t / \mathrm{cm}^{2}$ & $t / \mathrm{cm}^{2}$ & $t / \mathrm{cm}^{2}$ & $\%$ & $\%$ & $\%$ & $\mathrm{t} / \mathrm{cm}^{2}$ & \\
\hline 6 & $\begin{array}{l}0,289 \\
(1,0 \%)\end{array}$ & $\begin{array}{l}5,04 \\
(1,5 \%) \\
\end{array}$ & $\begin{array}{l}5,23 \\
(1,2 \%) \\
\end{array}$ & $\begin{array}{l}6,63 \\
(0,6 \%) \\
\end{array}$ & $\begin{array}{l}6,89 \\
(0,5 \%) \\
\end{array}$ & 2,2 & 13 & 56 & 2100 & 4 \\
\hline 8 & $\begin{array}{l}0,501 \\
(1,2 \%)\end{array}$ & $\begin{array}{l}5,57 \\
(1,4 \%)\end{array}$ & $\begin{array}{l}5,84 \\
(1,1 \%) \\
\end{array}$ & $\begin{array}{c}7,38 \\
(1,9 \%)\end{array}$ & $\begin{array}{l}7,72 \\
(1,6 \%) \\
\end{array}$ & 2,1 & 15 & 53 & 2100 & 4 \\
\hline 16 & $\begin{array}{l}1,97 \\
(0,4 \%)\end{array}$ & $\begin{array}{l}5,00 \\
(1,9 \%) \\
\end{array}$ & $\begin{array}{l}5,23 \\
(1,8 \%) \\
\end{array}$ & $\begin{array}{l}6,85 \\
(1,7 \%) \\
\end{array}$ & $\begin{array}{l}7,16 \\
(1,7 \%) \\
\end{array}$ & 1,7 & 14 & 52 & 2100 & 4 \\
\hline 18 & $\begin{array}{l}2,51 \\
(0,5 \%)\end{array}$ & $\begin{array}{l}5,09 \\
(1,8 \%) \\
\end{array}$ & $\begin{array}{l}5,33 \\
(1,9 \%) \\
\end{array}$ & $\begin{array}{l}7,03 \\
(1,9 \%) \\
\end{array}$ & $\begin{array}{l}7,37 \\
(1,8 \%) \\
\end{array}$ & 1,7 & 15 & 49 & 2100 & 4 \\
\hline 26 & $\begin{array}{l}5,16 \\
(0,1 \%)\end{array}$ & $\begin{array}{l}5,23 \\
(0,1 \%) \\
\end{array}$ & $\begin{array}{l}5,45 \\
(0,2 \%) \\
\end{array}$ & $\begin{array}{l}7,06 \\
(0,3 \%)\end{array}$ & $\begin{array}{l}7,39 \\
(0,3 \%)\end{array}$ & 1,5 & 14 & 46 & 2100 & 4 \\
\hline $12,7\left(1 / 2^{\prime \prime}\right)$ & $\begin{array}{l}0,935 \\
(0,1 \%)\end{array}$ & $\begin{array}{l}15,96 \\
(0,7 \%)\end{array}$ & $\begin{array}{l}16,45 \\
(0,8 \%)\end{array}$ & $\begin{array}{l}17,55 \\
(0,6 \%)\end{array}$ & $\begin{array}{l}18,35 \\
(0,6 \%)\end{array}$ & - & 2,3 & - & 1970 & 4 \\
\hline
\end{tabular}

Variationskoeffizient in Klommern

Tabelle 1: Festigkeitswerte des verwendeten BARO-Stahles $(\phi 6)$, BOX-ULTRA - Stahles $(\phi 8, \phi 16, \phi 18, \phi 26)$ und der VSL-Litzen $\left(1 / 2^{\prime \prime}\right)$

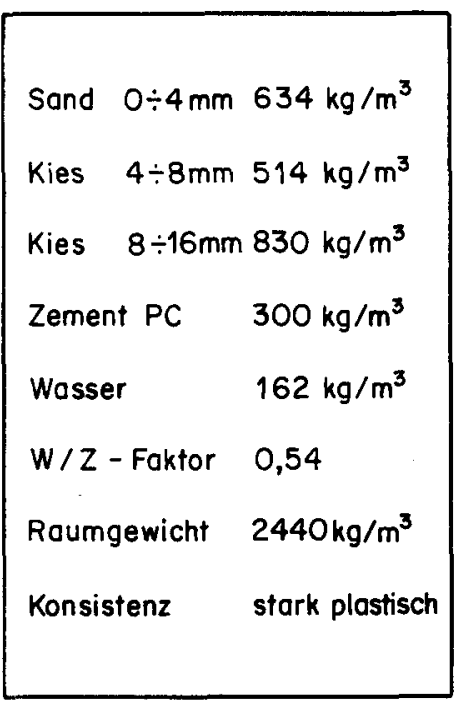

\begin{tabular}{|c|c|c|c|c|c|c|c|}
\hline Balken & $T W_{1}$ & $T W_{2}$ & $T W_{3}$ & $\mathrm{TW}_{4}$ & $T W_{5}$ & Mittelwert & Var. Koeff \\
\hline Alter(Toge) & 45 & 45 & 45 & 36 & 38 & & \\
\hline$\beta_{b z}\left(\mathrm{~kg} / \mathrm{cm}^{2}\right)$ & $\begin{array}{l}45 \\
46 \\
49\end{array}$ & $\begin{array}{l}45 \\
47 \\
51\end{array}$ & $\begin{array}{l}58 \\
49 \\
41\end{array}$ & $\begin{array}{l}50 \\
45 \\
50\end{array}$ & $\begin{array}{l}54 \\
46 \\
60\end{array}$ & 49 & $10,4 \%$ \\
\hline$\beta_{w}\left(\mathrm{~kg} / \mathrm{cm}^{2}\right)$ & $\begin{array}{l}410 \\
434 \\
434\end{array}$ & $\begin{array}{l}417 \\
410 \\
403\end{array}$ & $\begin{array}{l}393 \\
392 \\
386\end{array}$ & $\begin{array}{l}458 \\
445 \\
444\end{array}$ & $\begin{array}{l}374 \\
392 \\
379\end{array}$ & 411 & $6,4 \%$ \\
\hline$\beta_{\mathrm{p}}\left(\mathrm{kg} / \mathrm{cm}^{2}\right)$ & $\begin{array}{l}331 \\
329\end{array}$ & $\begin{array}{l}326 \\
331\end{array}$ & $\begin{array}{l}313 \\
299\end{array}$ & $\begin{array}{l}337 \\
357\end{array}$ & $\begin{array}{l}292 \\
338\end{array}$ & 325 & $5,9 \%$ \\
\hline$E_{5-100}\left(t / \mathrm{cm}^{2}\right)$ & $\begin{array}{l}344 \\
327\end{array}$ & $\begin{array}{l}323 \\
312\end{array}$ & - & $\begin{array}{l}349 \\
352\end{array}$ & $\begin{array}{l}343 \\
333\end{array}$ & 335 & $4,2 \%$ \\
\hline
\end{tabular}

Tabelle 2: Zusammenset- Tabelle 3: Festigkeitswerte der Betonprismen zung des Frischbetons $12 \times 12 \times 36$ im Versuchsalter der Balken

\begin{tabular}{|c|c|c|c|c|c|c|c|c|c|c|c|}
\hline Prismo & $\begin{array}{c}\text { Alter } \\
\mathrm{Tg}\end{array}$ & $\begin{array}{c}\dot{\varepsilon}=\Delta \varepsilon / \Delta t \\
10^{-3} / \mathrm{min}\end{array}$ & $\begin{array}{c}\mathrm{t}_{\mathrm{w}} \\
\mathrm{min}\end{array}$ & $\begin{array}{c}\beta_{\mathrm{pd}} \\
\mathrm{kg} / \mathrm{cm}^{2}\end{array}$ & $\begin{array}{c}\beta_{\mathrm{ps}}\left(2^{\prime}\right) \\
\mathrm{kg} / \mathrm{cm}^{2}\end{array}$ & $\begin{array}{c}\beta_{p s}\left(30^{\prime}\right) \\
\mathrm{kg} / \mathrm{cm}^{2}\end{array}$ & $\frac{\beta_{\mathrm{ps}}\left(2^{\prime}\right)}{\beta_{p d}}$ & $\frac{\beta_{\mathrm{ps}}\left(30^{\prime}\right)}{\beta_{\mathrm{pd}}}$ & $\begin{array}{c}E_{\mathrm{b}(5 \div 100} \\
t / \mathrm{cm}^{2}\end{array}$ & $\begin{array}{c}\varepsilon_{\mathrm{u}} \\
10^{-3}\end{array}$ & Prisma \\
\hline 1 & 46 & 0,2 & 2 & 287 & 253 & & 0,88 & & 301 & 1,40 & 1 \\
2 & 45 & 0,2 & 2 & 268 & 235 & & 0,88 & & 274 & 1,52 & 2 \\
3 & 45 & 0,2 & 2 & 275 & 238 & & 0,87 & & 272 & 1,76 & 3 \\
4 & 42 & 0,2 & 2 & 308 & 271 & & 0,88 & & 284 & 1,54 & 4 \\
5 & 38 & 0,2 & 2 & 286 & 251 & & 0,88 & & 271 & 1,44 & 5 \\
\hline
\end{tabular}

1) Wert aus früheren Versuchen übernommen

Tabelle 4: Festigkeitswerte der Beton-Probekörper 


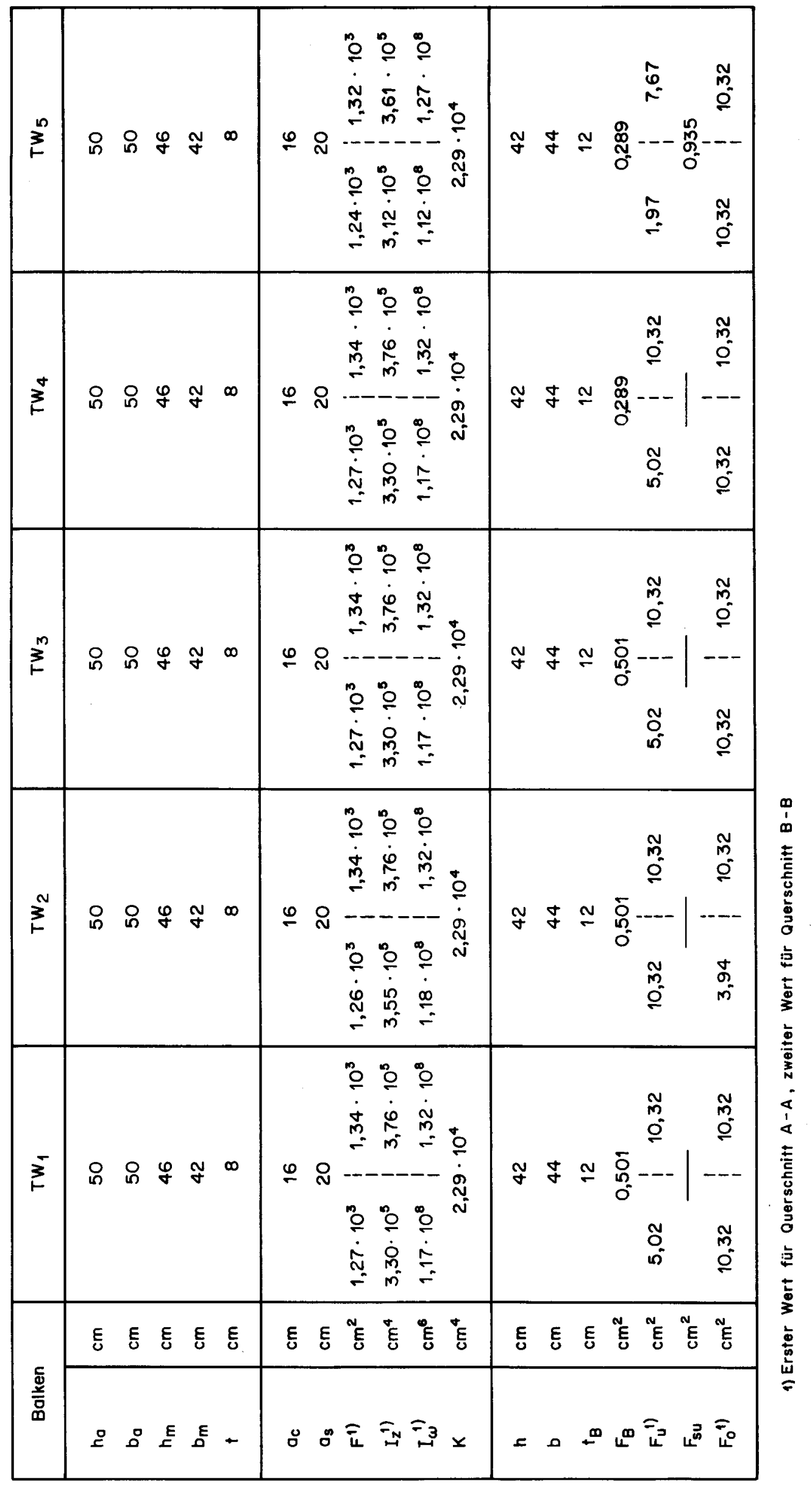

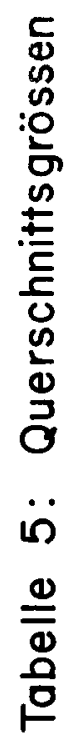


Homogener Zustand: Gemischte Torsion $k=\sqrt{\frac{G K \cdot L^{2}}{E I_{\omega}}}=3,20$

\begin{tabular}{|l|c|c|c|c|c|}
\hline \multirow{2}{*}{ Bolken } & $Q_{z}(g)$ & $T_{W}(g)$ & $T_{s}(g)$ & $M_{2}(g)$ & $M_{\omega}(g)$ \\
\cline { 2 - 6 } & $t$ & $\mathrm{~cm} \dagger$ & $\mathrm{cm} \dagger$ & $\mathrm{cm} \dagger$ & $\mathrm{cm}^{2} \dagger$ \\
\hline$T W_{1}, T W_{2}$ & 0,29 & 7,4 & 2,3 & 70 & 720 \\
\hline$T W_{3}, T W_{4}, T W_{5}$ & 0,26 & 2,0 & 0,7 & 66 & 200 \\
\hline
\end{tabular}

1) Schnittkrufte im Querschnitt B-B

Bruchzustand: Wölbtorsion

\begin{tabular}{|l|c|c|c|c|}
\hline \multirow{2}{*}{ Balken } & $Q_{z}(g)$ & $T_{W}(g)$ & $M_{z}(g)$ & $M_{\omega}(g)$ \\
\hline$T W_{1}, T W_{2}$ & $\dagger$ & $c m t$ & $c m t$ & $\mathrm{~cm}^{2} t$ \\
\hline$T W_{3}, T W_{4}, T W_{5}$ & $-0,44$ & $-9,7$ & 50 & 870 \\
\hline
\end{tabular}

2) Schnittkrőfte im Querschnitt A-A

Tabelle 6: Schnittkräfte aus Eigengewicht des Balkens und des Belastungsarmes

\begin{tabular}{|c|c|c|c|c|c|c|}
\hline \multirow{2}{*}{ Balken } & \multicolumn{2}{|c|}{ Gemessen } & \multicolumn{2}{|c|}{ Gerechnet } & \multirow{2}{*}{$\frac{P_{f E}^{E x}}{P_{u}^{T h}}$} & \multirow{2}{*}{$\frac{P_{u E}^{E x}}{P_{u}^{T h}}$} \\
\hline & $P_{f E}^{E x}$ & $P_{U E}^{E X}$ & $P_{u}^{T h}$ & $\frac{1}{\operatorname{tg} \alpha}$ & & \\
\hline$T W_{1}$ & 4,60 & 5,70 & 4,00 & vorne 0,99 & 1,15 & 1,43 \\
\hline$T W_{2}$ & 5,00 & 6,25 & 4,35 & hinten 0,80 & 1,15 & 1,44 \\
\hline$T W_{3}$ & 8,50 & 10,45 & 7,40 & vorne 0,99 & 1,15 & 1,41 \\
\hline$T W_{4}$ & 7,40 & 9,60 & 6,50 & vorne 1,68 & 1,14 & 1,48 \\
\hline$T W_{5}$ & 7,20 & 9,60 & $\begin{array}{c}\left(6,35^{31}\right) \\
\left.6,60^{4}\right)\end{array}$ & vorne $\begin{array}{c}(1,64) \\
1,70\end{array}$ & $\begin{array}{c}(1,13) \\
1,09\end{array}$ & $\begin{array}{c}(1,51) \\
1,45\end{array}$ \\
\hline
\end{tabular}

3) Theor. Werte mit Streckgrenze des Sponnstahles berechnet

4) Theor. Werte mit gemessener Dehnung des Spannstahles berechnet

Tabelle 7: Gemessene und theoretische Bruchlasten des Scheibenmodells 


\begin{tabular}{|c|c|c|c|c|c|c|c|}
\hline \multirow{5}{*}{$\stackrel{N}{i}$} & 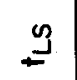 & 高 & 용 \& & $\stackrel{2}{2}=$ & 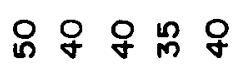 & $=$ 은 & 왕ㅇㅇㅇㅇㅇ \\
\hline & $\frac{8}{8}$ & 1 & 0 운 & $0 n$ & $\infty \infty \sim \infty \sigma$ & $0+$ & $\sigma a$ 을 \\
\hline & $a^{\omega}$ & - & 员㙕品 & 용 \& & 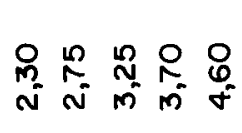 & 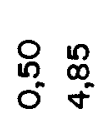 & 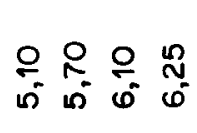 \\
\hline & $a^{\varangle}$ & $\leftarrow$ & 용요 & $\begin{array}{l}0 \\
\text { ho } \\
0 \\
0\end{array}$ & 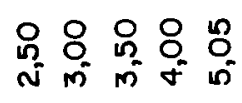 & 象员 & 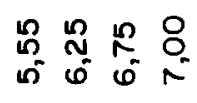 \\
\hline & ن) & & $-N m \sigma$ & $\infty 6$ & $\sim \infty \sigma \circ=$ & $\stackrel{N}{\sim}$ & $\stackrel{\square}{\square} \mathscr{F}$ \\
\hline
\end{tabular}

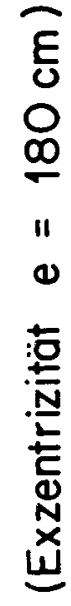

$\sum_{5}^{2}$

\begin{tabular}{|c|c|c|c|c|c|c|c|}
\hline \multirow{5}{*}{$\sum^{5}$} & 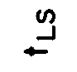 & 高 & 용우 웅 & ㅇ ก & $\stackrel{m}{m} \tilde{m}$ & 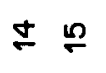 & 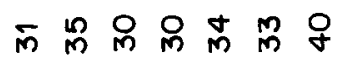 \\
\hline & $\frac{8}{8}$ & 1 & 0 으른 & $0 \infty$ & $\infty \sim r$ & 00 & $\infty \infty \infty \sigma a=$ 응 \\
\hline & $a^{w}$ & + & 용요용 용 & 罩 & 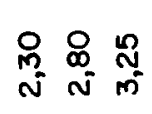 & 员 怘 & 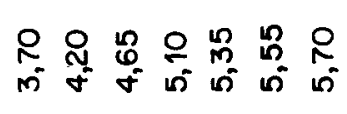 \\
\hline & $a^{\alpha}$ & - & 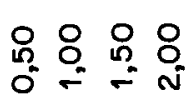 & 菖 8 & $\begin{array}{lll}8 & 8 & 8 \\
\text { N } & 8 \\
\text { m } & \text { m }\end{array}$ & 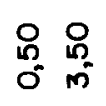 & 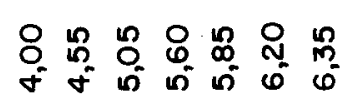 \\
\hline & $\dot{j}$ & & $-n m a$ & $\infty 0$ & $\sim \infty \sigma$ & 우 둔 & 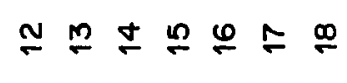 \\
\hline
\end{tabular}

$\sum$

高

岇

음

$\infty$

$\stackrel{0}{\bar{\nu}}$
믐 


\begin{tabular}{|c|c|c|c|c|c|c|c|}
\hline \multirow{5}{*}{$\sum^{n}$} & $\stackrel{9}{-}$ & $\cdot \frac{\varrho}{\varepsilon}$ & 品品罢 & ำ ㅇำ & 우앙ㅇ & $\stackrel{5}{2}$ & ケ $q$ 思 \\
\hline & $\frac{8}{8}$ & 1 & $\circ$ 우 $\sim$ 은 & $0 \%$ & 우 0 으 & $O N$ & $\infty \stackrel{\circ}{=}$ \\
\hline & $a^{w}$ & - & 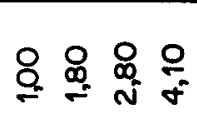 & 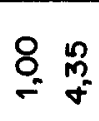 & 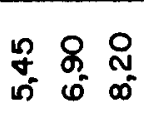 & \begin{tabular}{l}
8 \\
\hdashline \\
\hdashline
\end{tabular} & 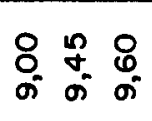 \\
\hline & $a^{4}$ & - & 웅 웡 & $\underset{8}{8}$ & 员 & 웅 & 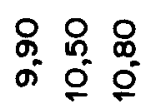 \\
\hline & $\dot{j}$ & & $-N m \sigma$ & 06 & $\wedge \infty \theta$ & 우 $=$ & $\simeq \stackrel{m}{\square}$ \\
\hline
\end{tabular}

8

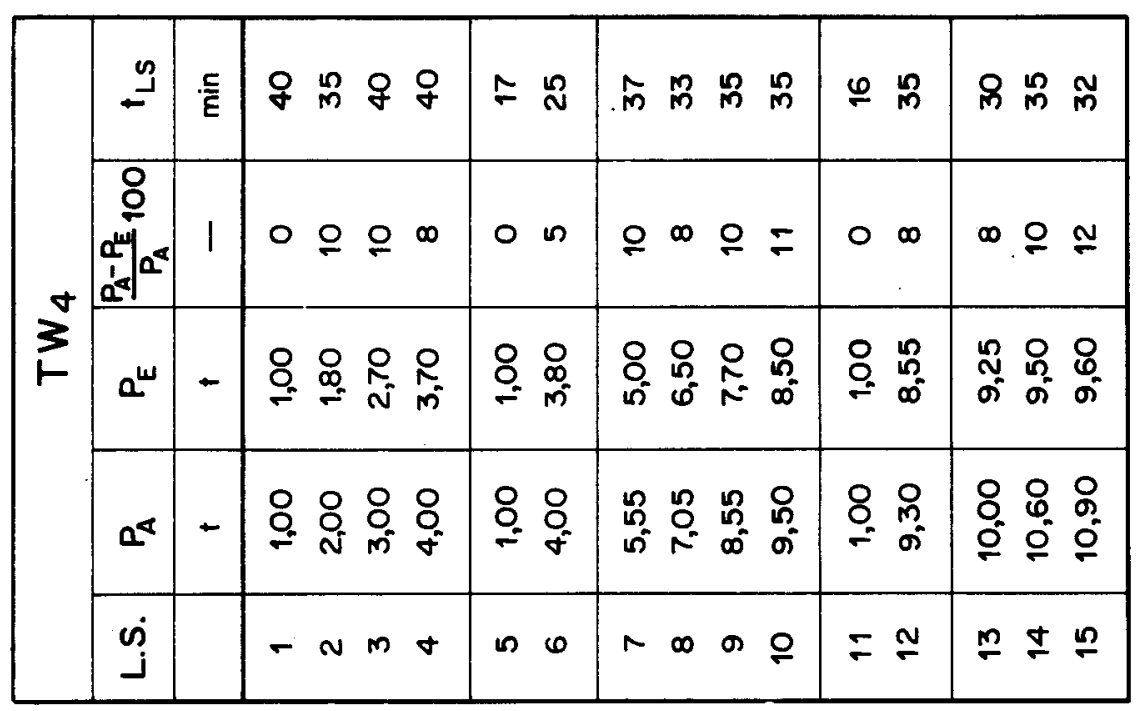

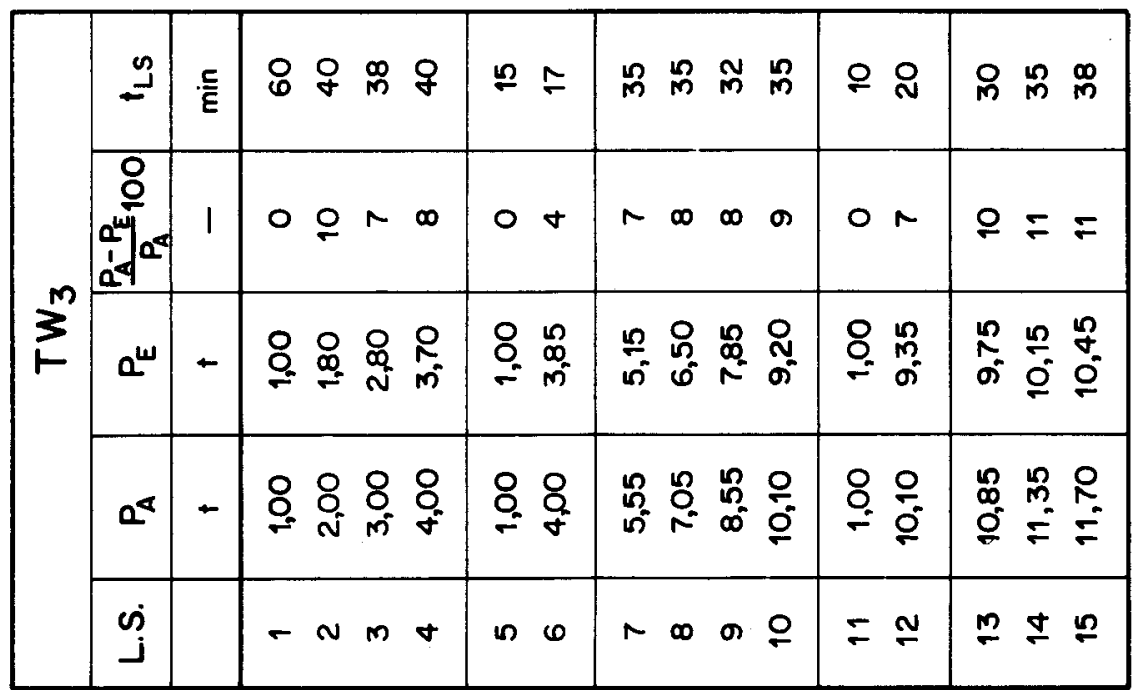




\begin{tabular}{|c|c|c|c|c|}
\hline Bolken & $\begin{array}{c}\text { Risslost } \\
P_{R}\end{array}$ & $\sigma_{R}{ }^{l}$ & $\beta_{W}$ & $\sigma_{R} / \sqrt{\beta_{W}}$ \\
\hline & $t$ & $\mathrm{~kg} / \mathrm{cm}^{2}$ & $\mathrm{~kg} / \mathrm{cm}^{2}$ & \\
\hline$T W_{1}$ & 1,30 & 52,6 & 426 & 2,55 \\
$T W_{2}$ & 1,40 & 55,0 & 410 & 2,72 \\
$T W_{3}$ & 2,50 & 54,7 & 390 & 2,76 \\
$T W_{4}$ & 2,40 & 52,8 & 449 & 2,50 \\
$T W_{5}$ & 4,50 & 43,2 & 382 & 2,22 \\
\hline
\end{tabular}

9) Vergleichsspannungen im Querschnitt B-B : Für TW, bis $T W_{4}$ vorne unten, für $T W_{5}$ hinten oben.

Tabelle 9: Vergleichsspannungen bei Rissbeginn 


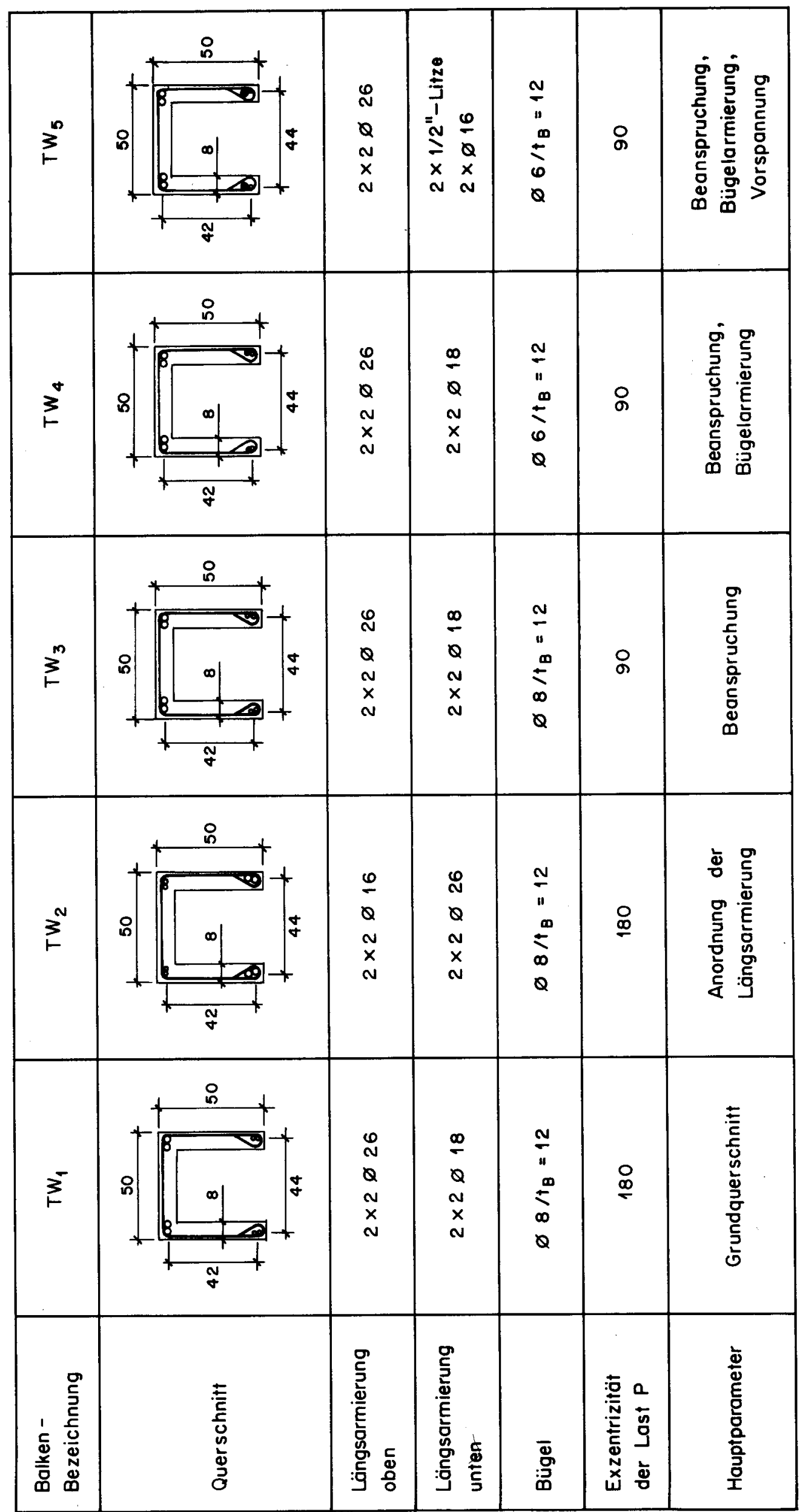



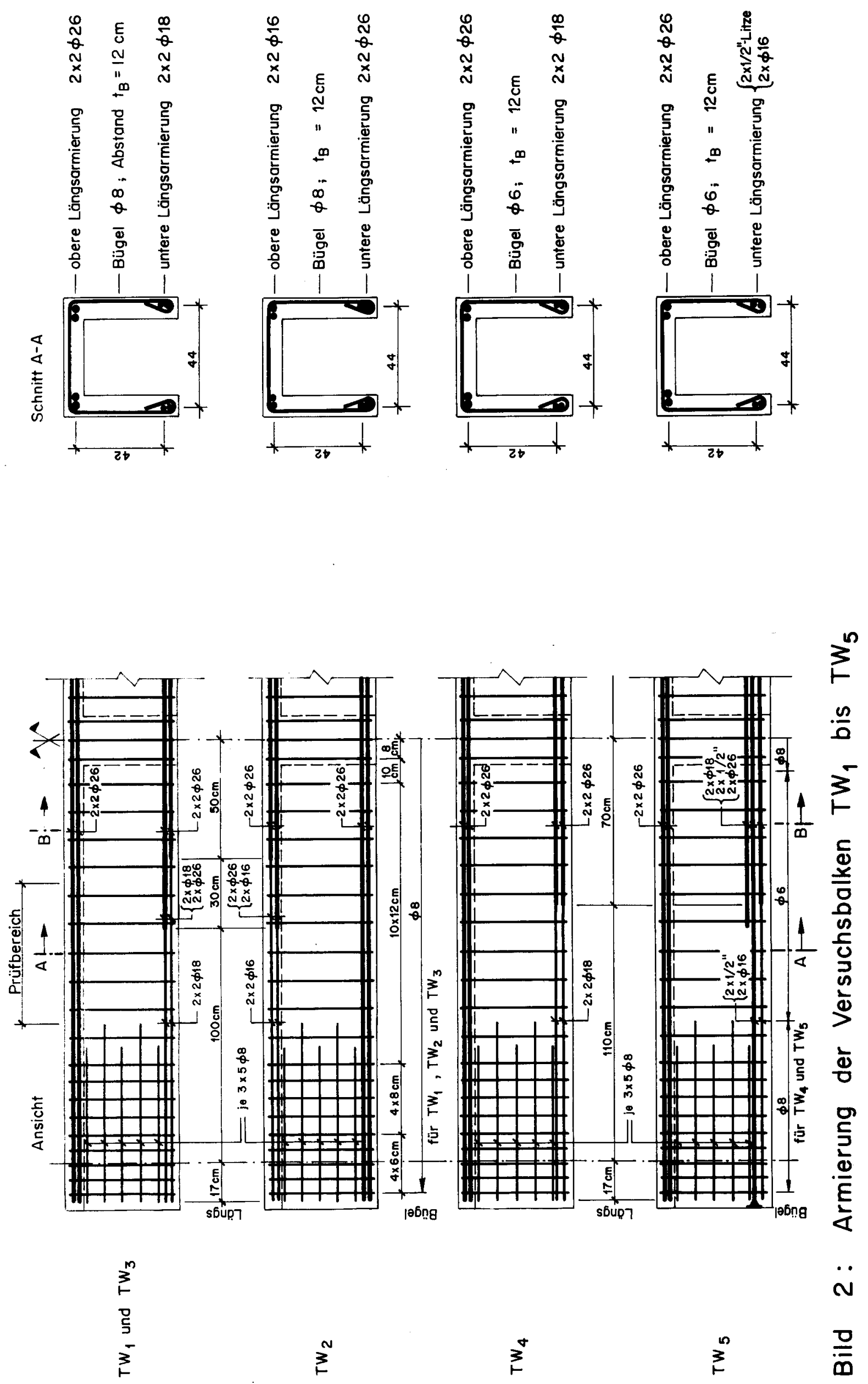


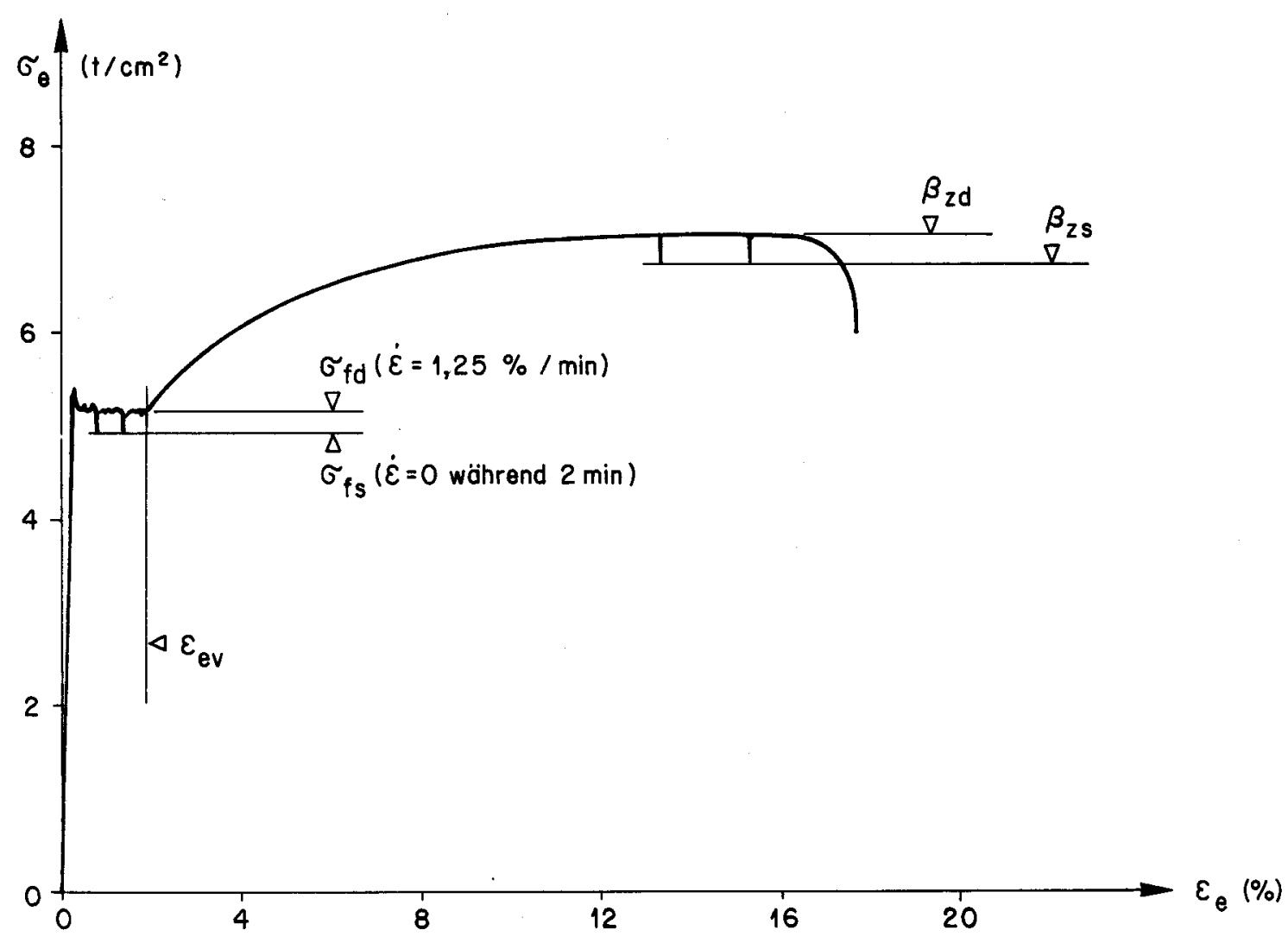

Bild 3: Spannungs-Dehnungs-Diagramm eines BOX-Stahles $\varnothing 16$

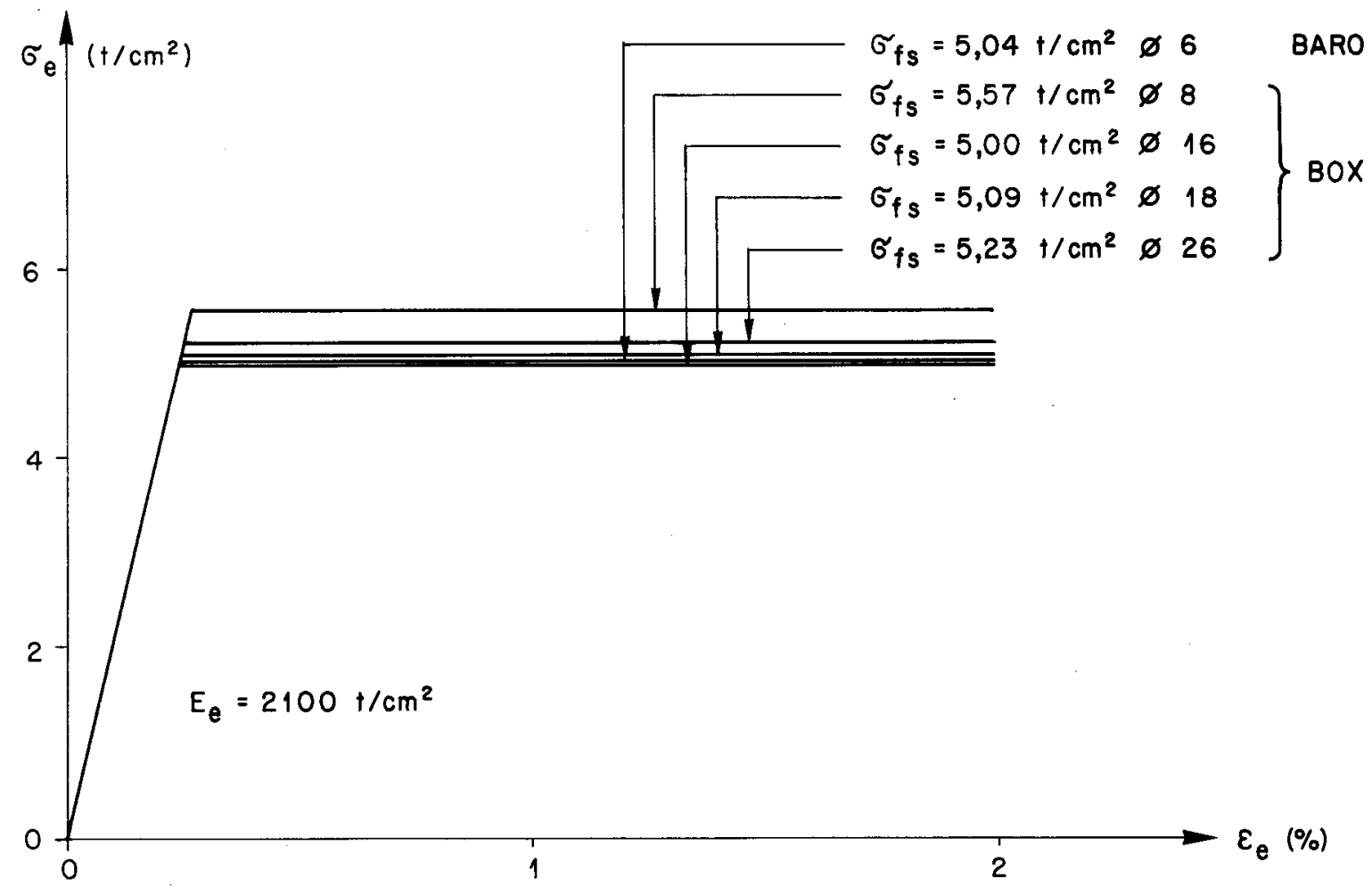

Bild 4: Gemittelte Spannungs-Dehnungs-Diagramme für Auswertung 


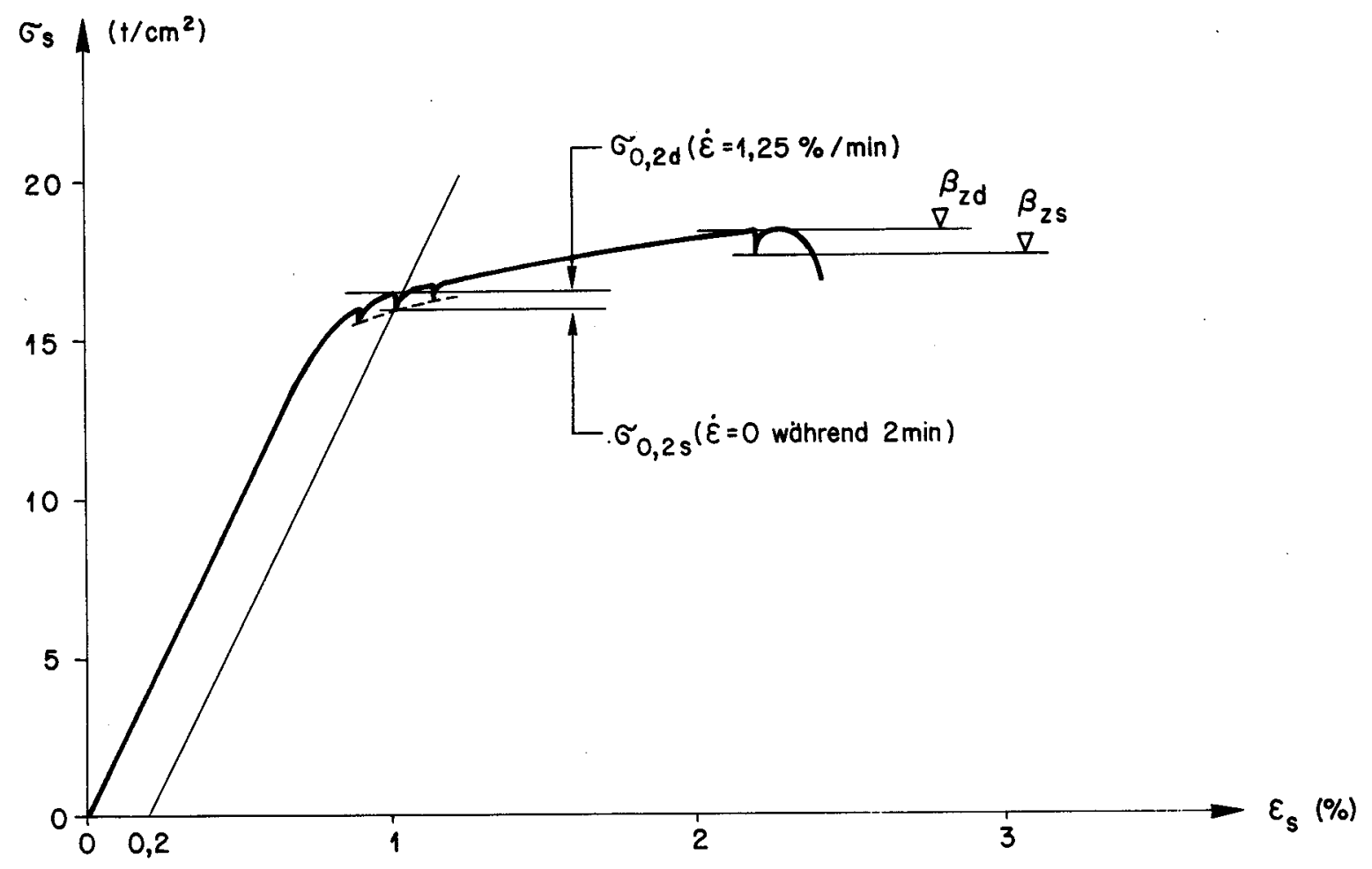

Bild 5 : Spannungs-Dehnungs-Diagramm einer VSL-Liłze $\varnothing 1 / 2 "$

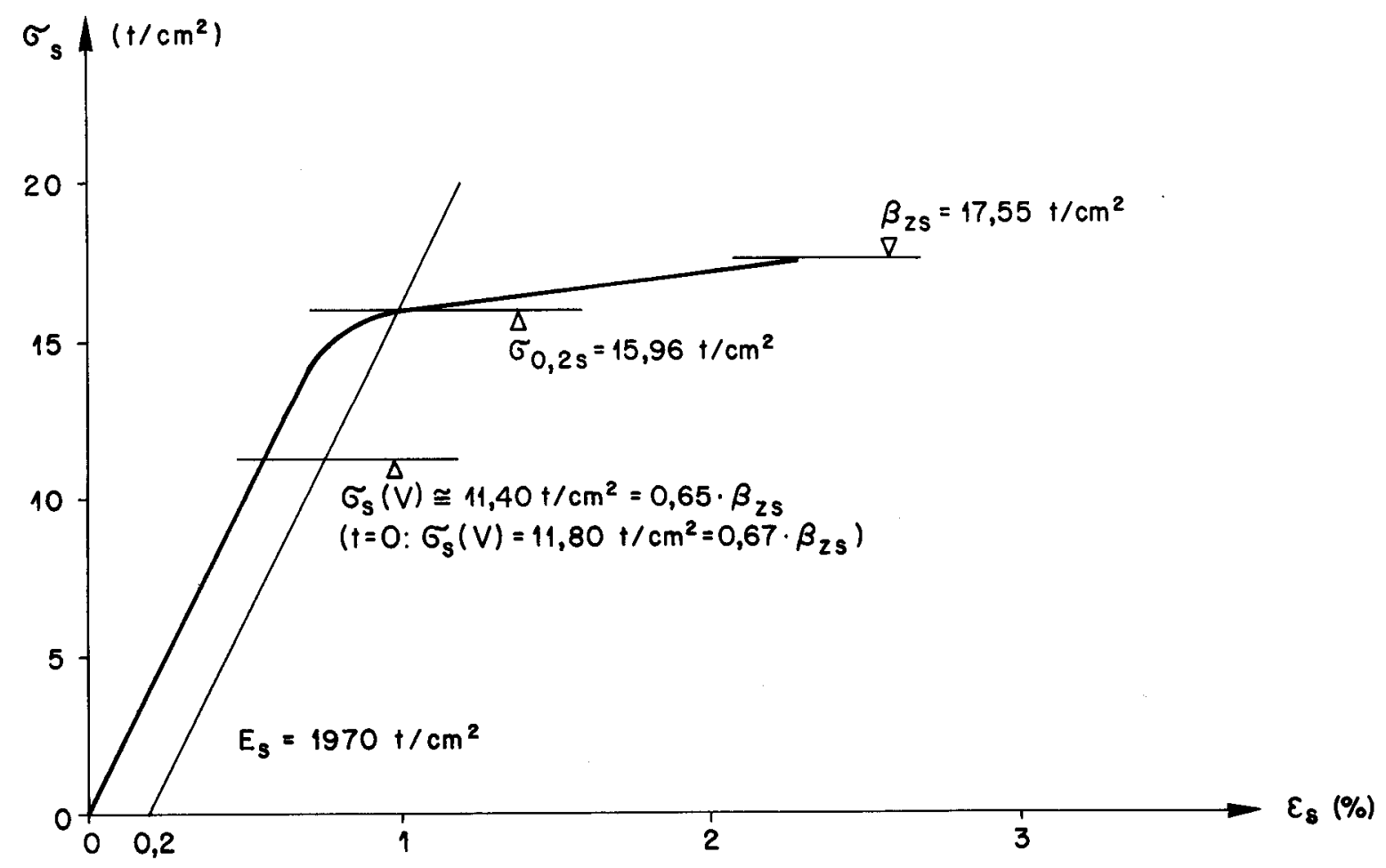

Bild 6 : Gemitteltes Spannungs-Dehnungs- Diagramm der Litzen für Auswertung 
40

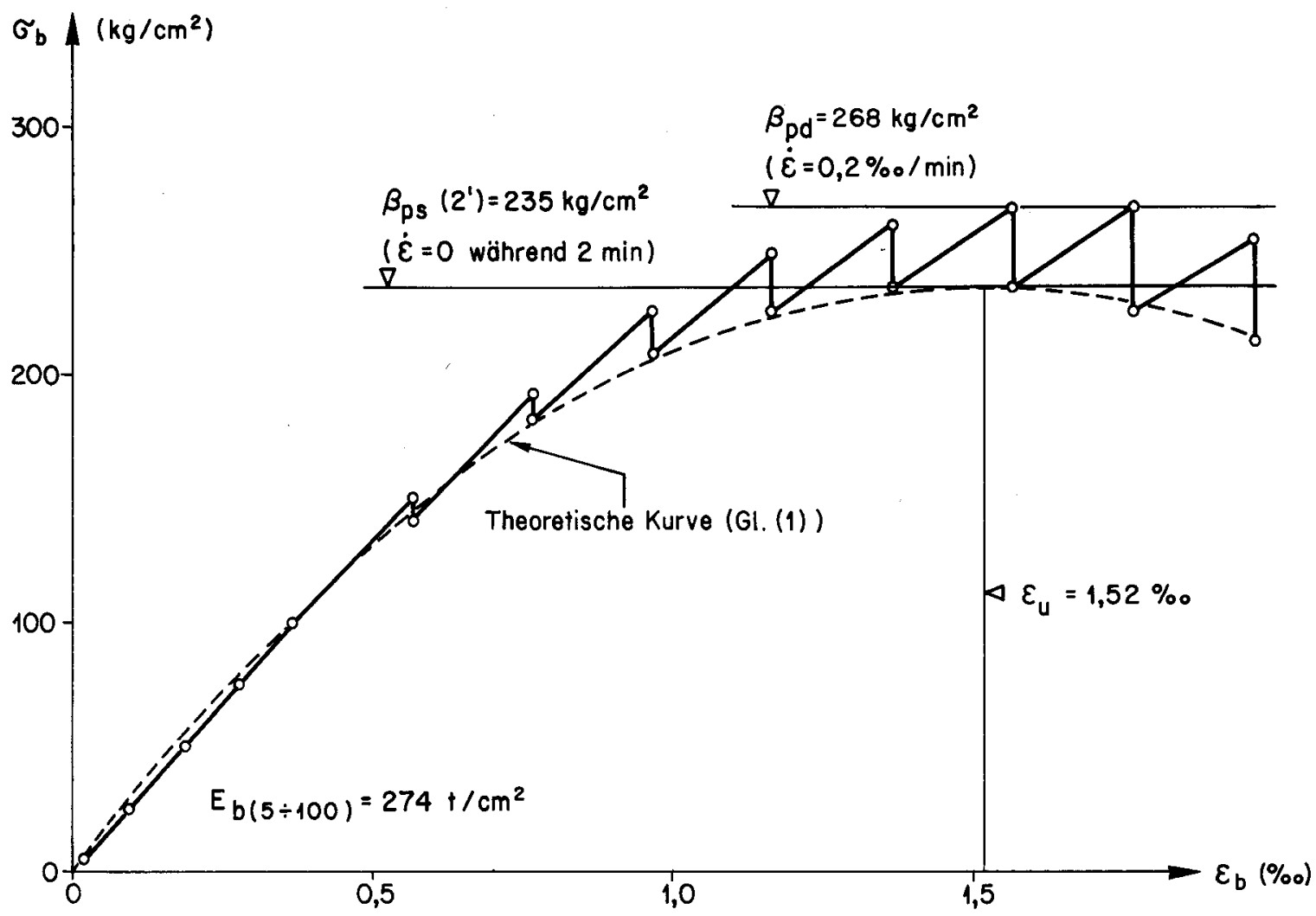

Bild 7: Spannungs-Dehnungs-Diagramm eines Betonprismas $\left(\mathrm{TW}_{2}\right)$

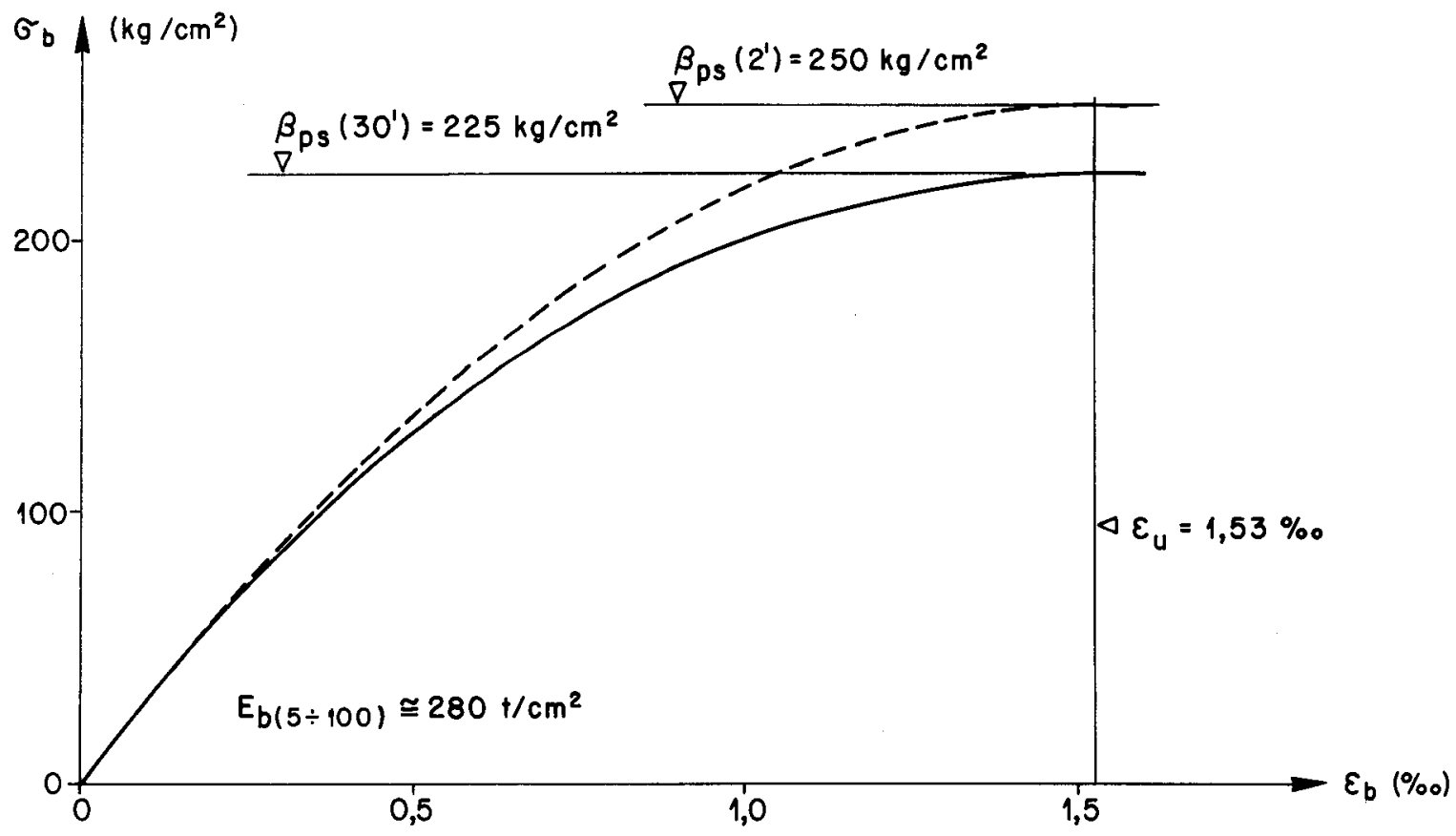

Bild 8 : Gemitteltes Spannungs-Dehnungs-Diagramm der Betonprismen für Auswertung 


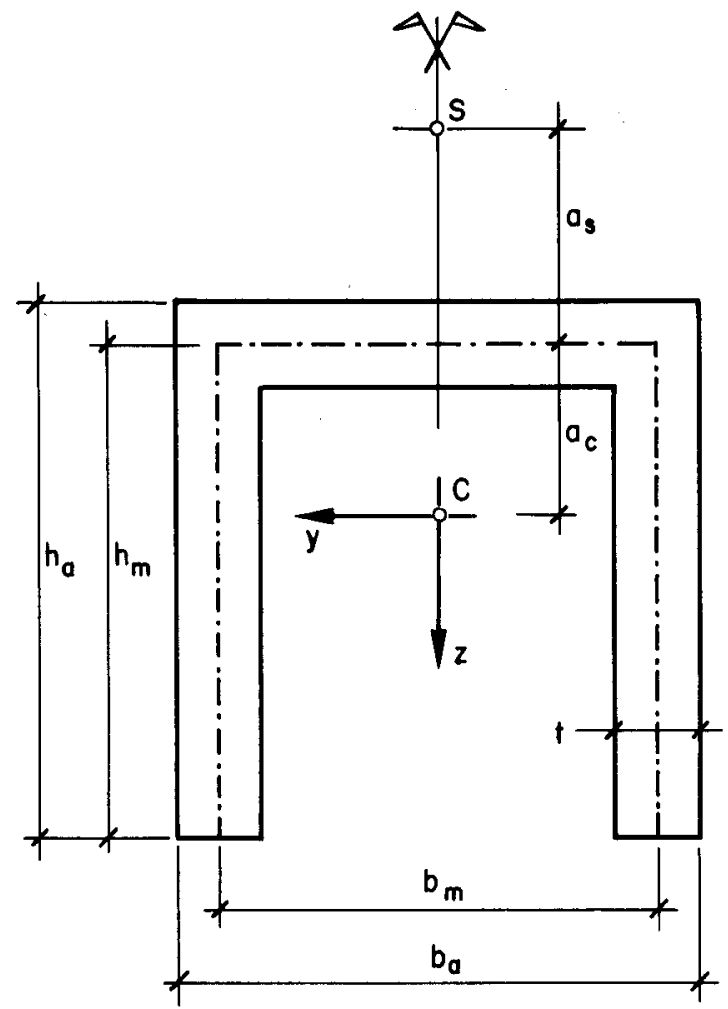

C : Schwerpunkt

$y, z$ : Schweraxen

$S$ : Schubmittelpunkt

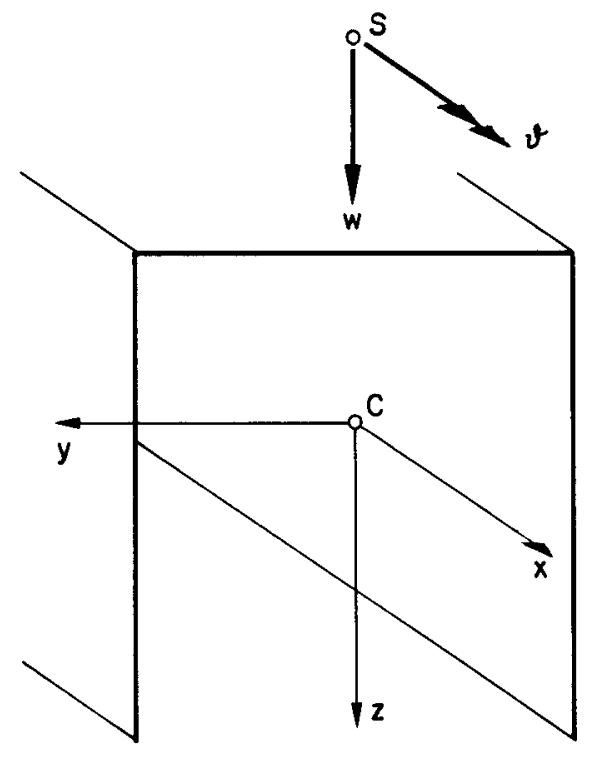

Verschiebungen

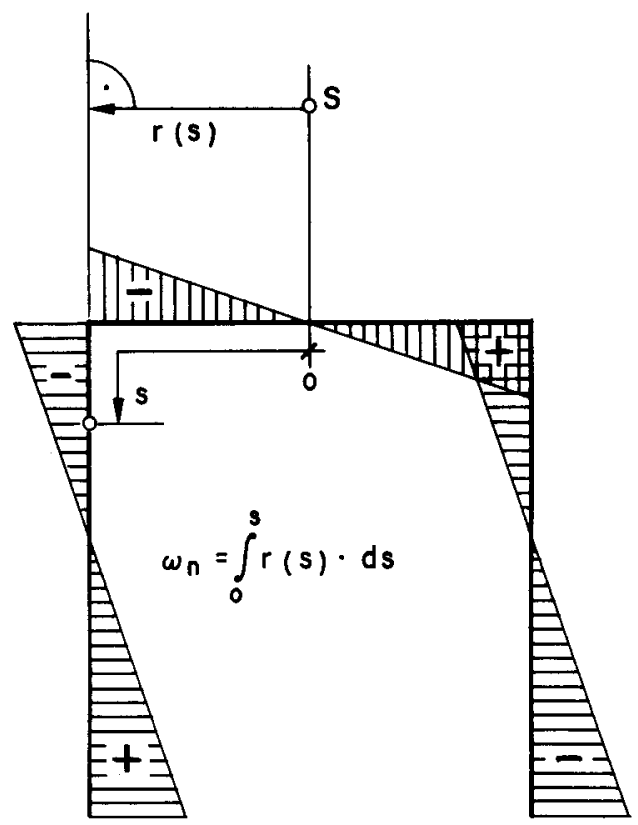

$\omega_{n}$ : Normierte, ouf den Schubmittelpunkt $S$ bezogene sektorielle Koordinate

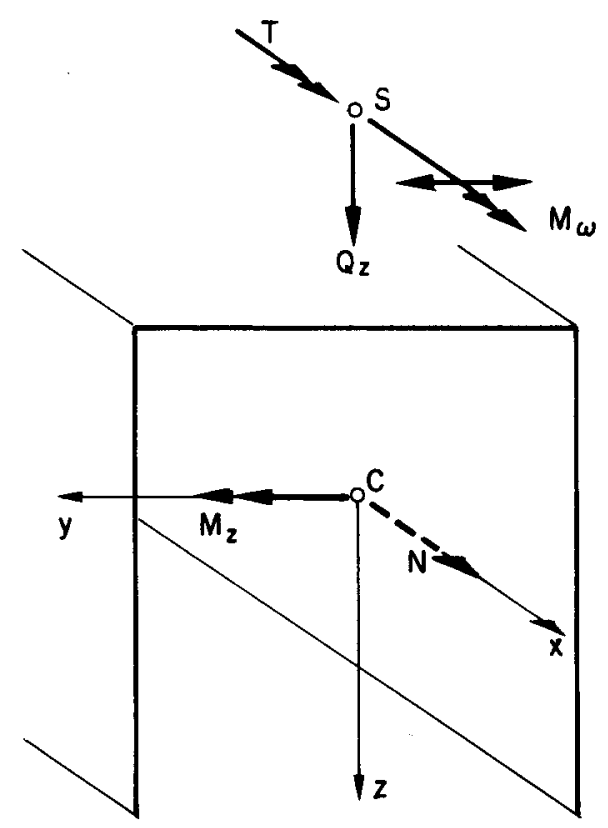

Schnittkräfte

Bild 9: Bezeichnungen am ungerissenen Querschnitt 

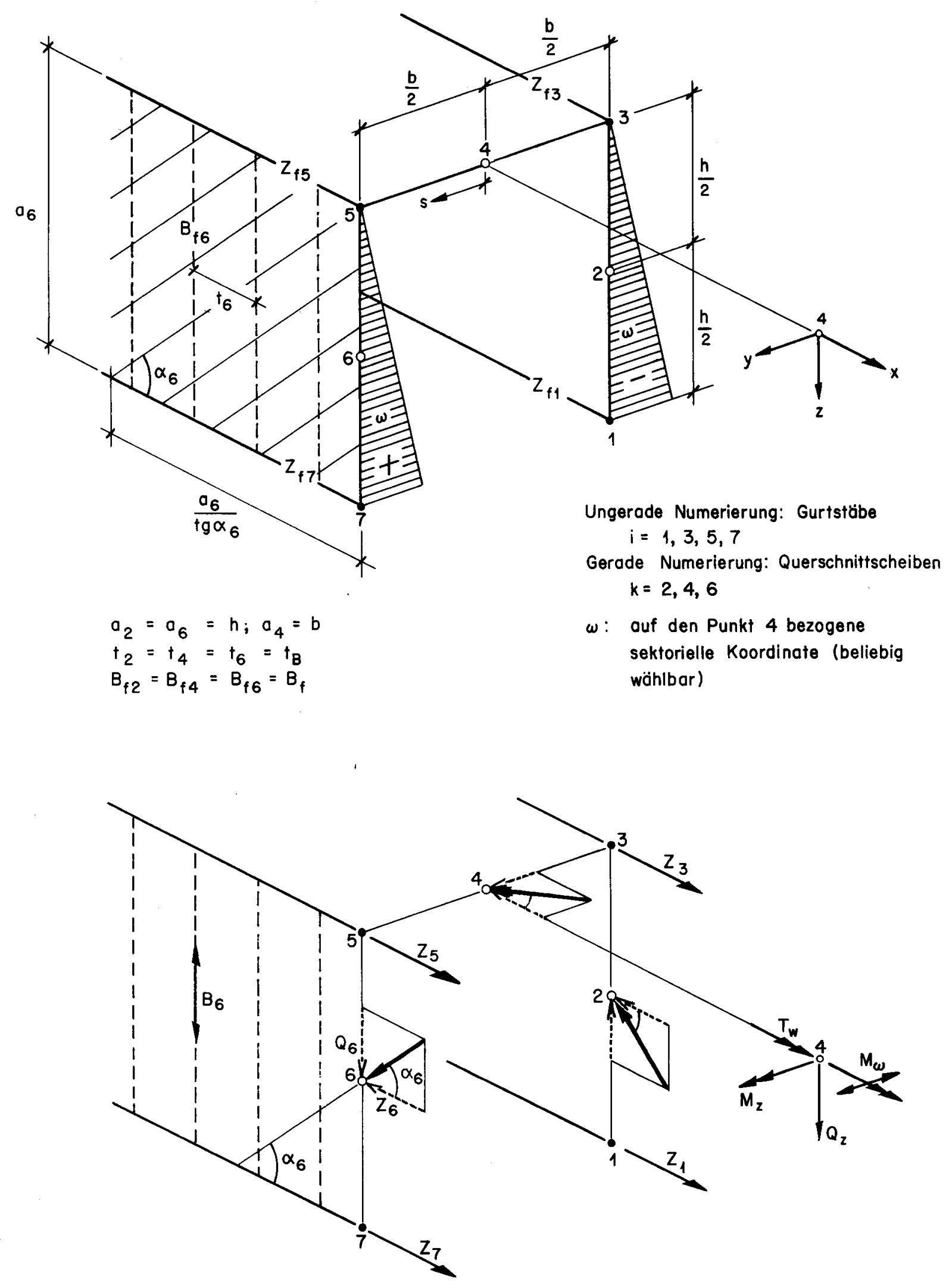

$Q_{k}=B_{k} \cdot \frac{a_{k}}{t_{k}} \cdot \frac{1}{\operatorname{tg} \alpha_{k}}$

$z_{k}=B_{k} \cdot \frac{a_{k}}{t_{k}} \cdot \frac{1}{\operatorname{tg}^{2} \alpha_{k}}$

Bild 10: Bezeichnungen am Bruchmodell 

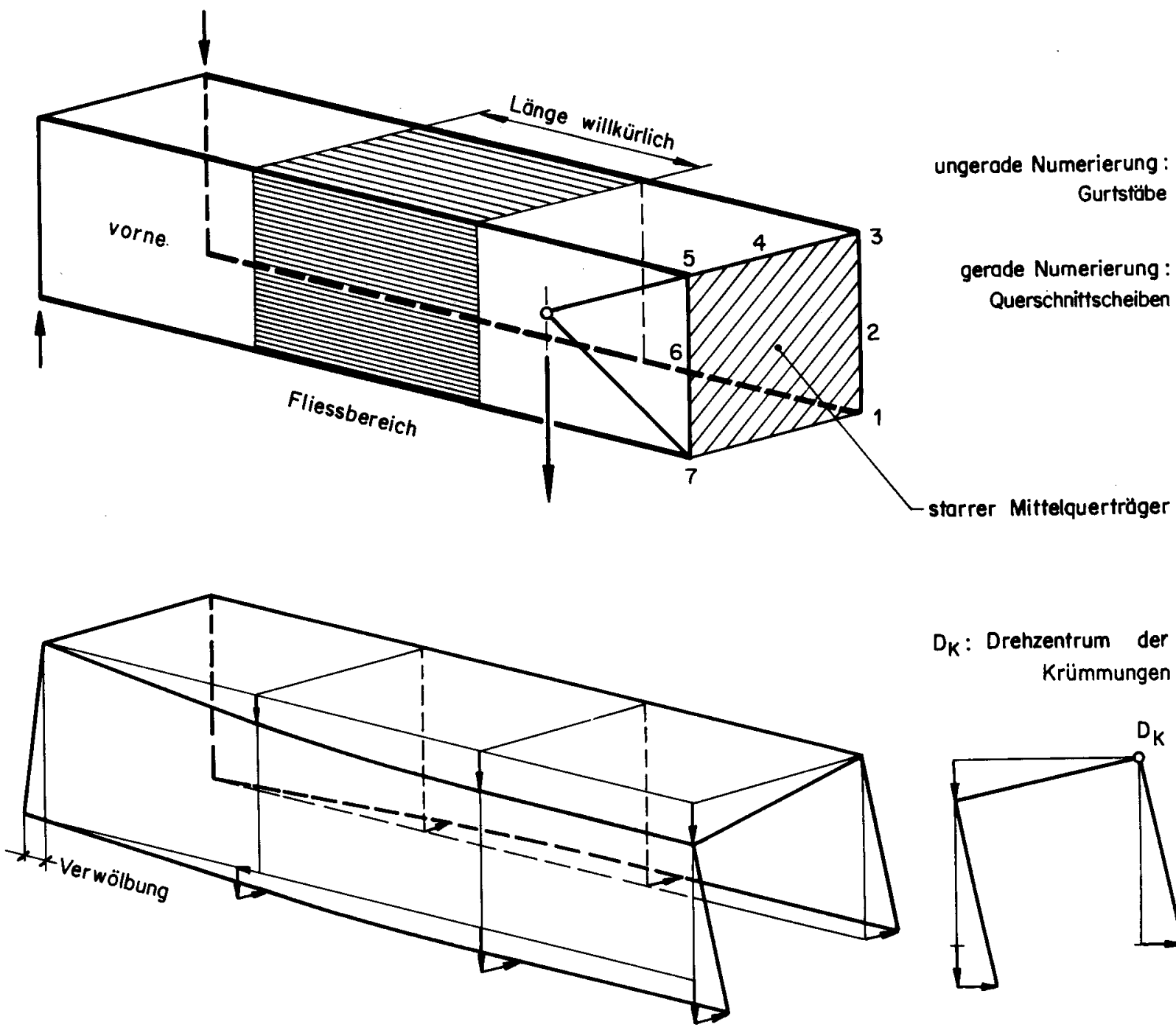

$D_{K}:$ Drehzentrum der Krümmungen

Krümmung der Querschnittscheibe 6 (Fliessen von Gurtstab 7)

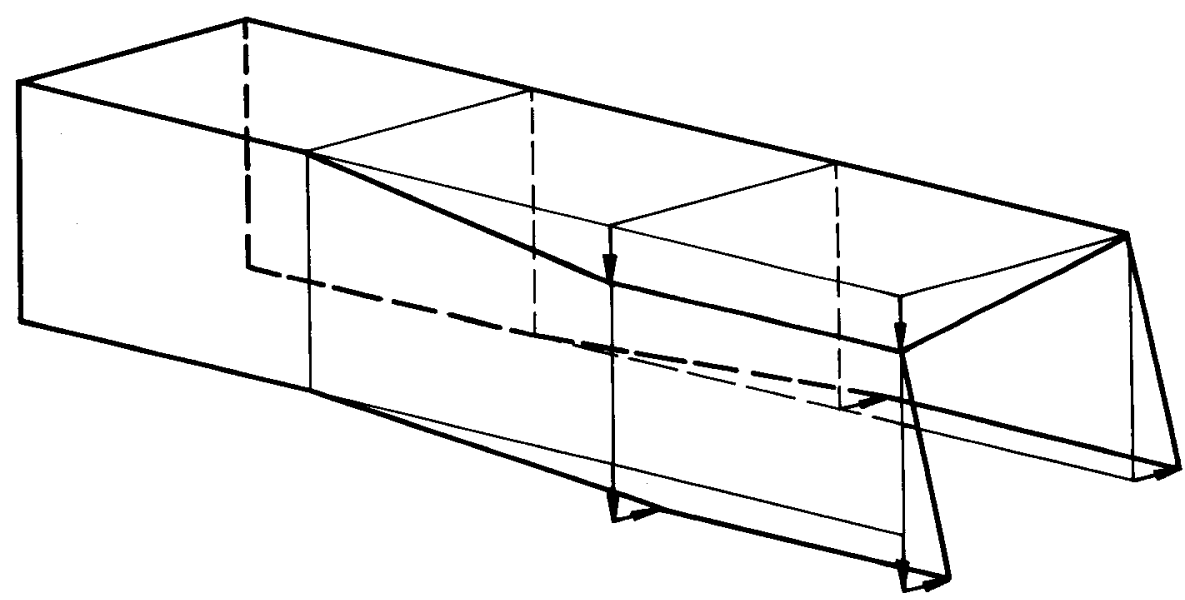

$D_{S}:$ Drehzentrum der Schiebungen

Schiebung der Querschittscheibe 6

Bild 11: Mechanismus beim Versagen der Balkenvorderseite 

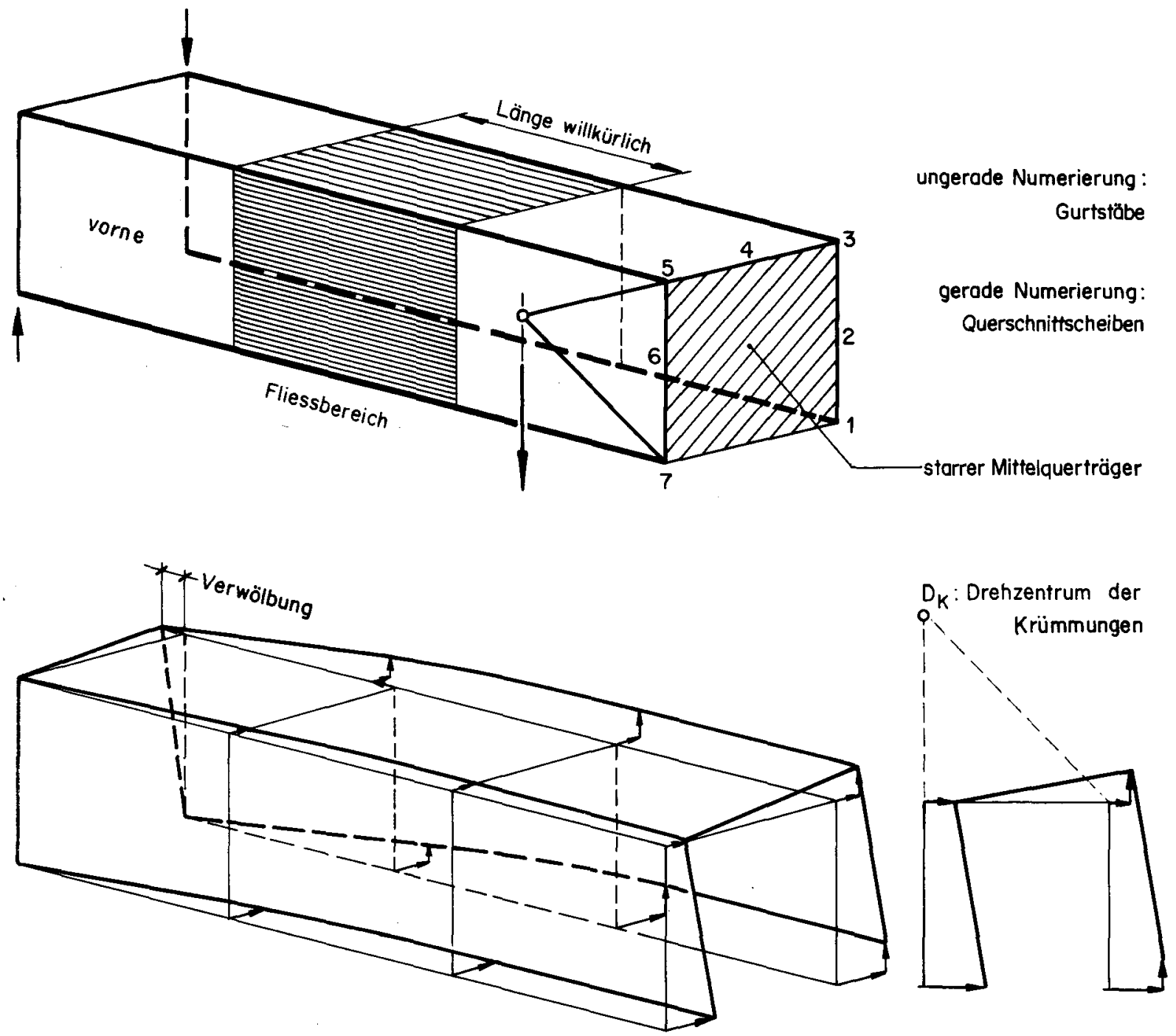

Krümmung der Querschnitłscheiben 2 und 4 (Fliessen von Gurtstab 3 )

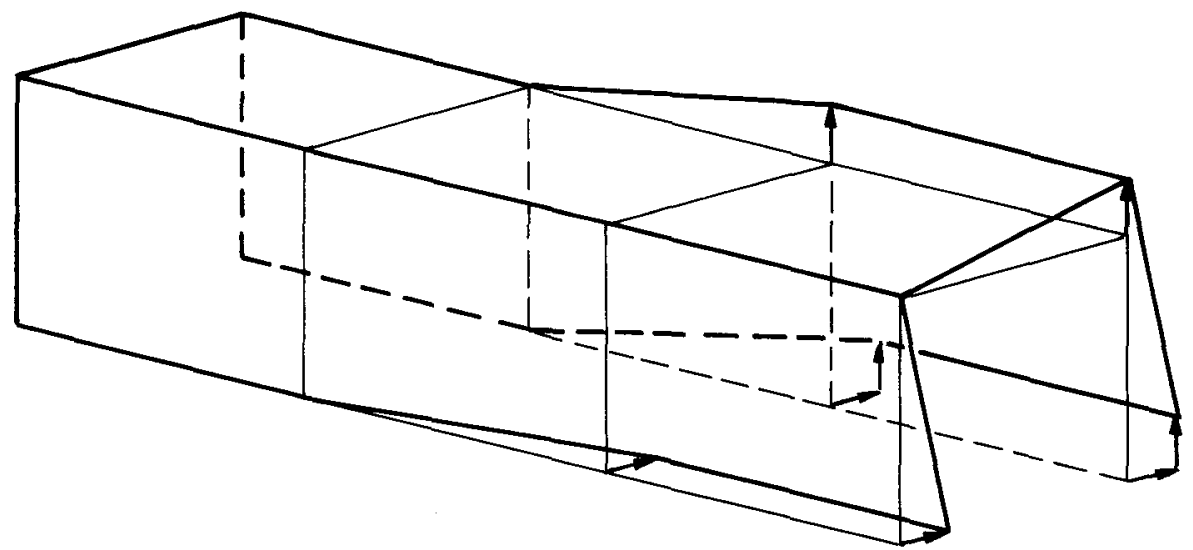

$D_{S}:$ Drehzentrum der Schiebungen

Schiebung der Querschnittscheibe 2

Bild 12: Mechanismus beim Versagen der Balkenhinterseite 


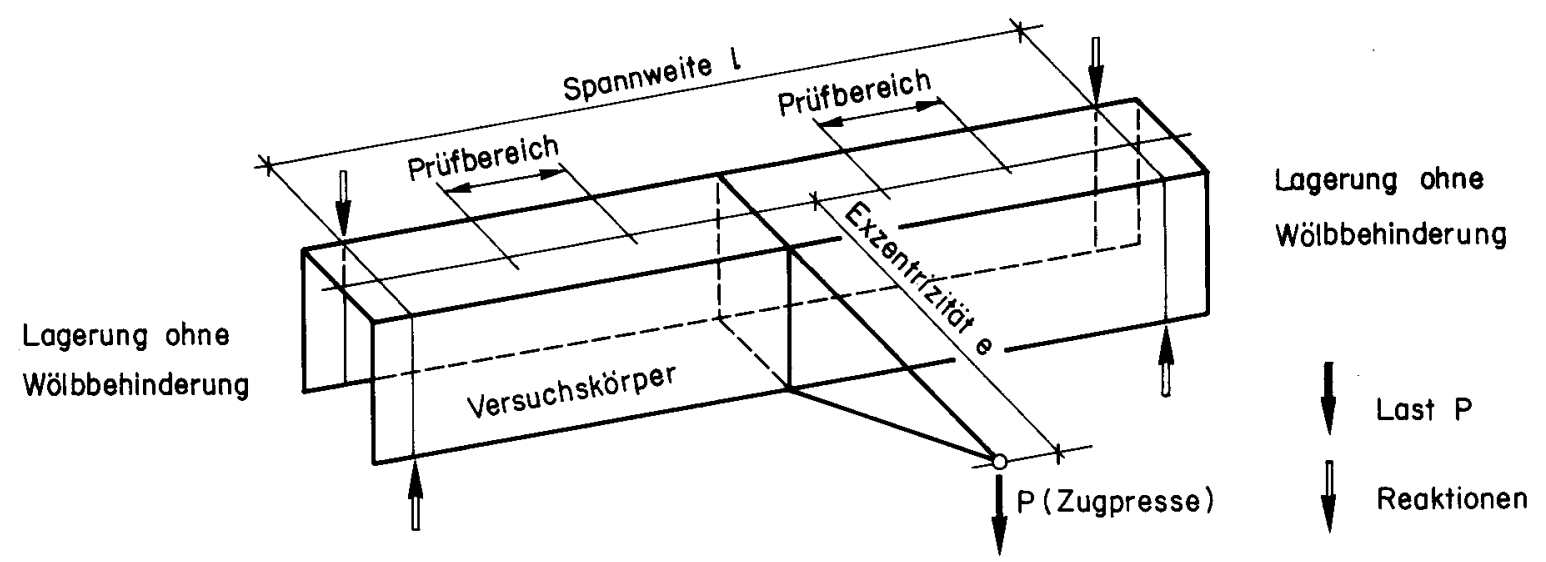

Bild 13: Schema der Versuchsanlage
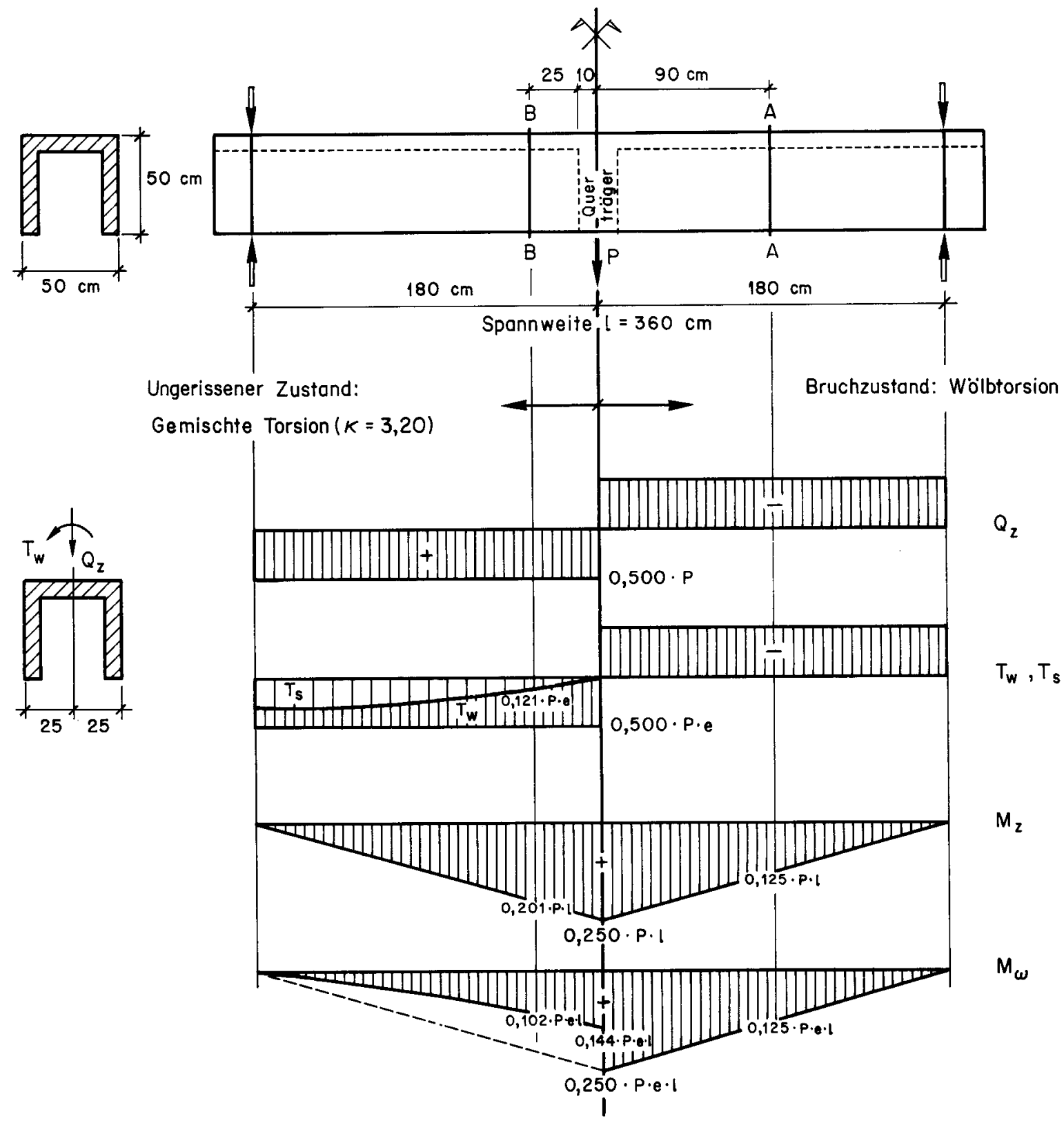

Bild 14: Theoretisch angenommene Schnittkräfte 


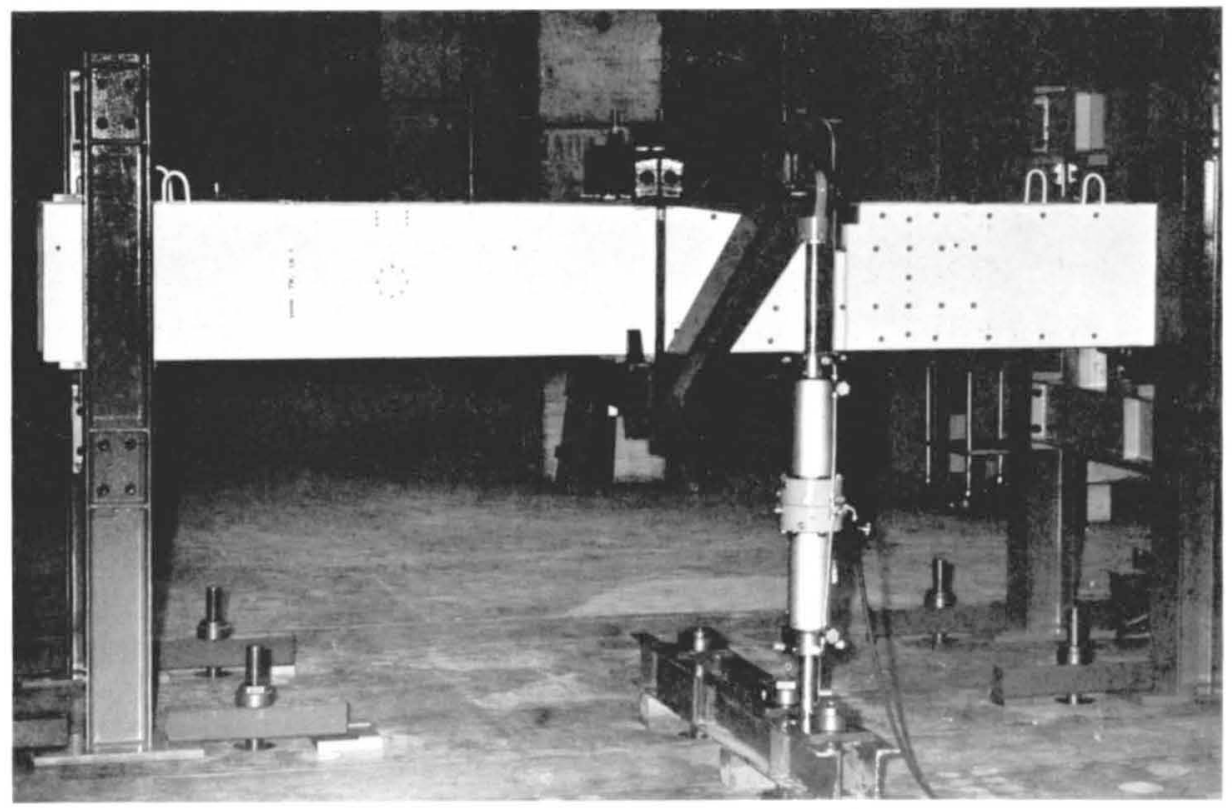

Bild 15: Gesamtansicht der Versuchsanlage

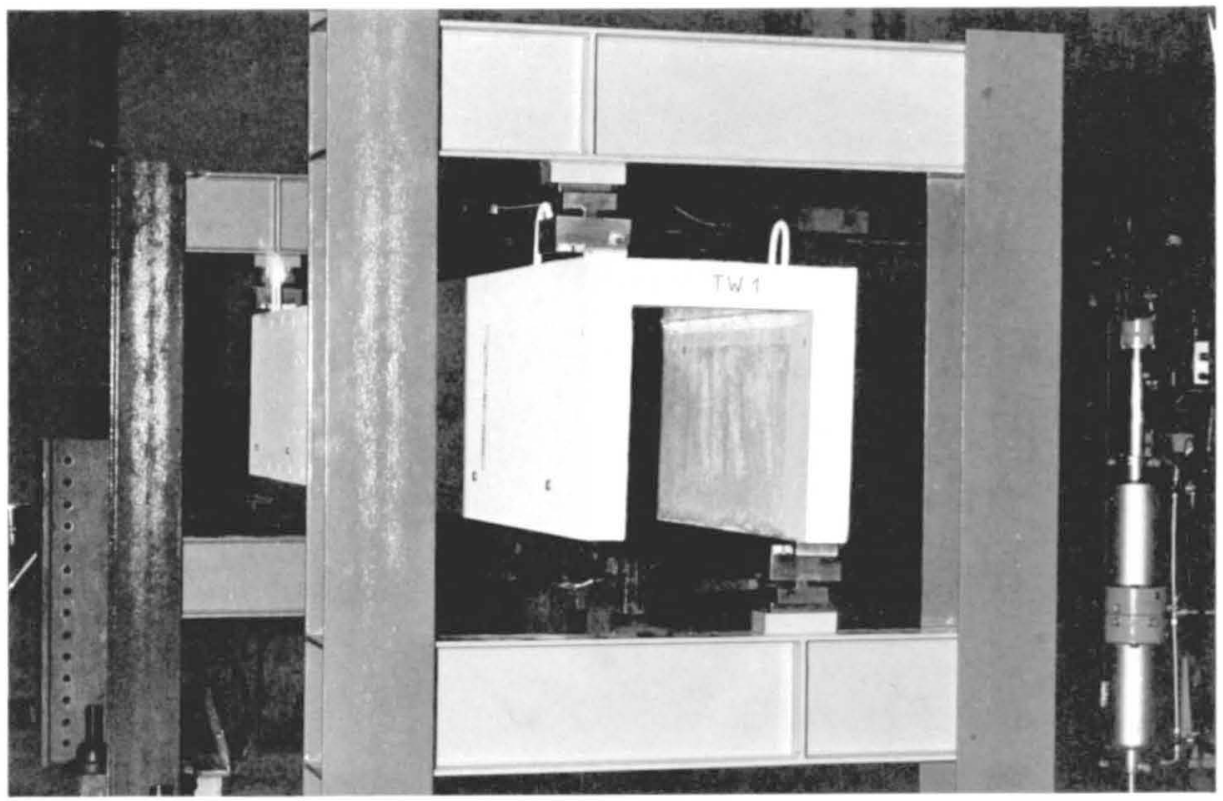

Bild 16: Auflagerrahmen der Versuchsanlage 


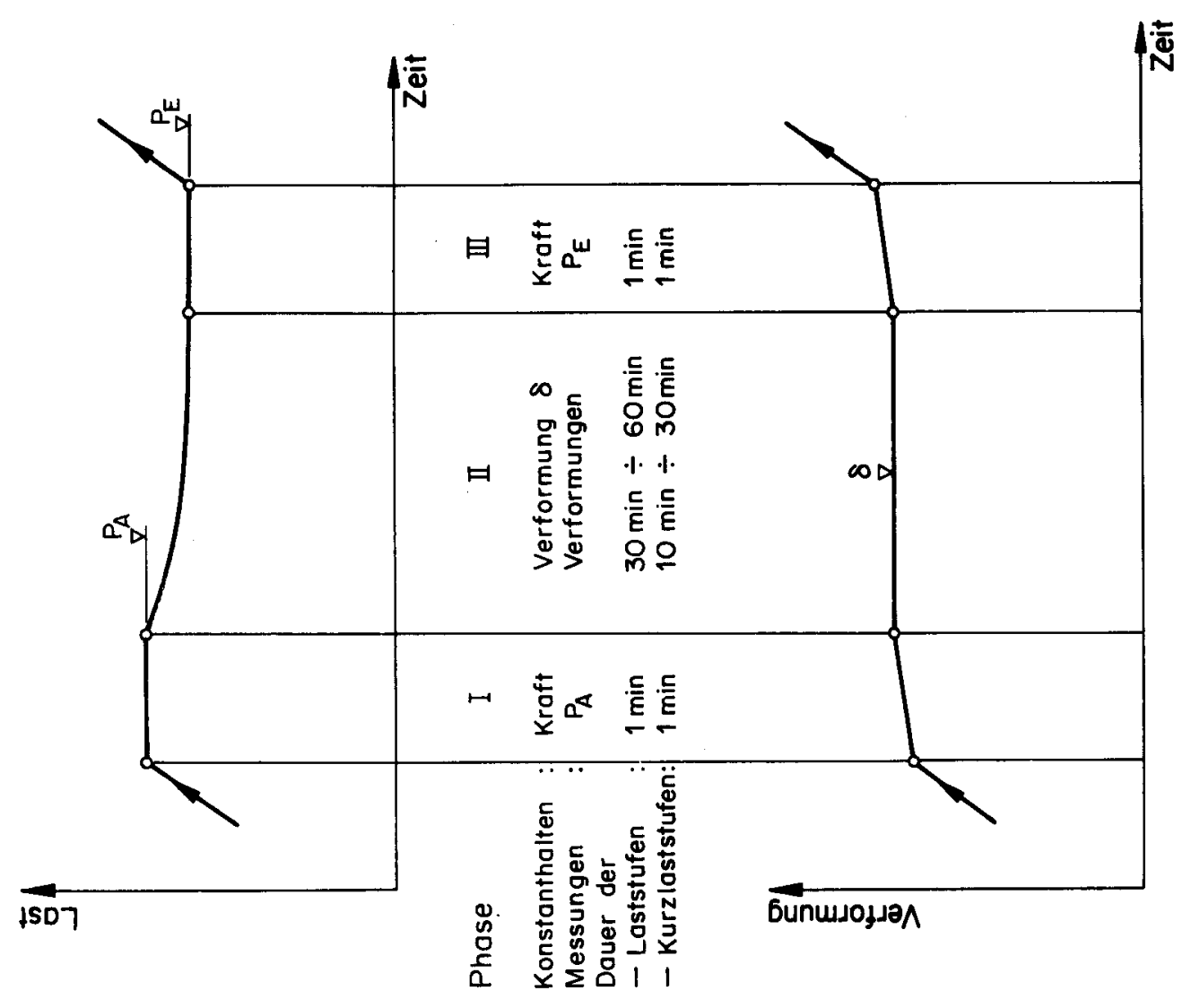

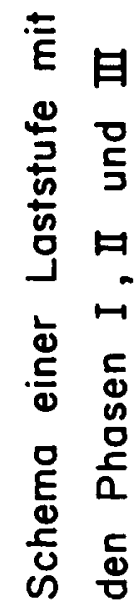

$\frac{\ddot{\infty}}{\ddot{0}}$

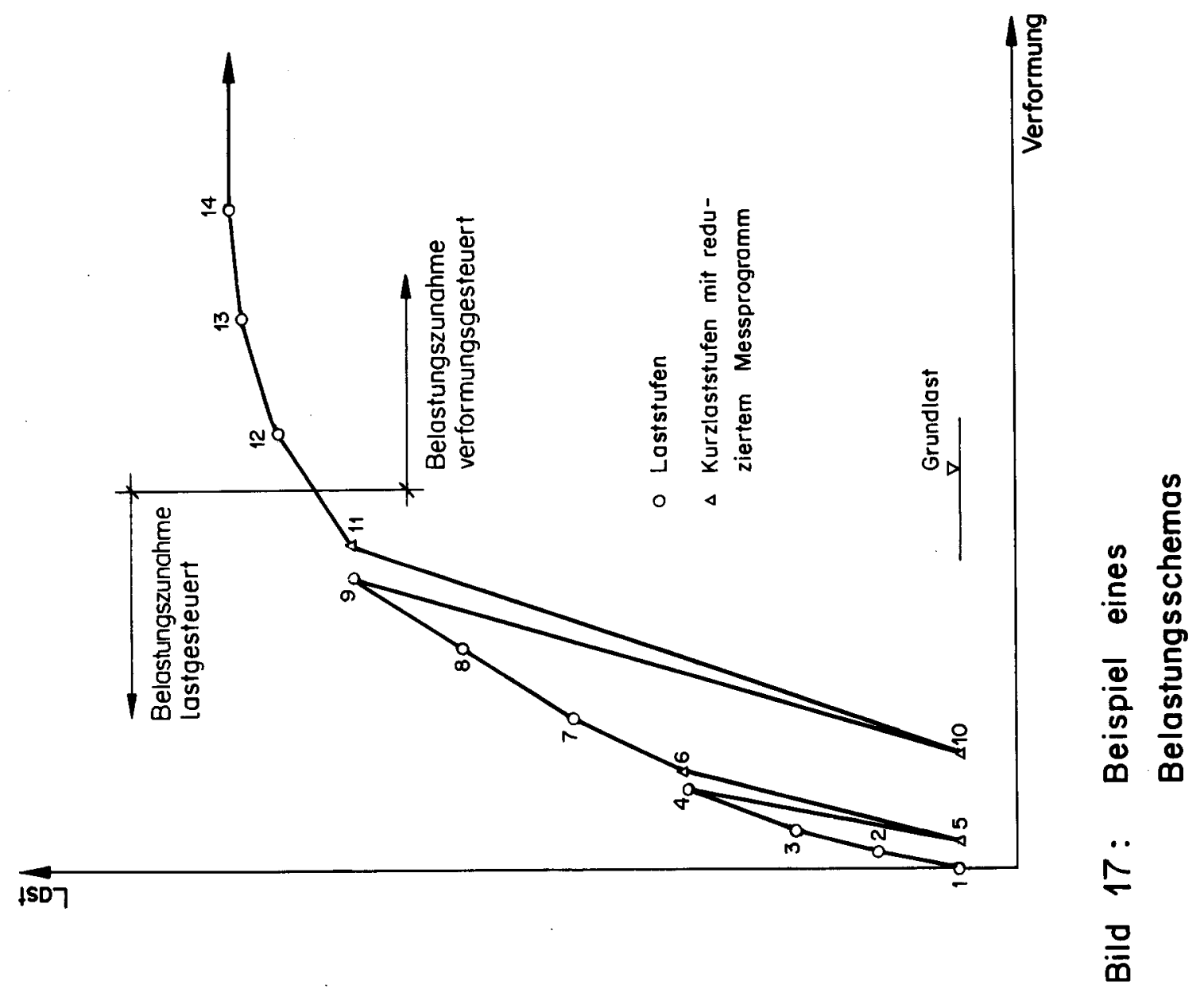




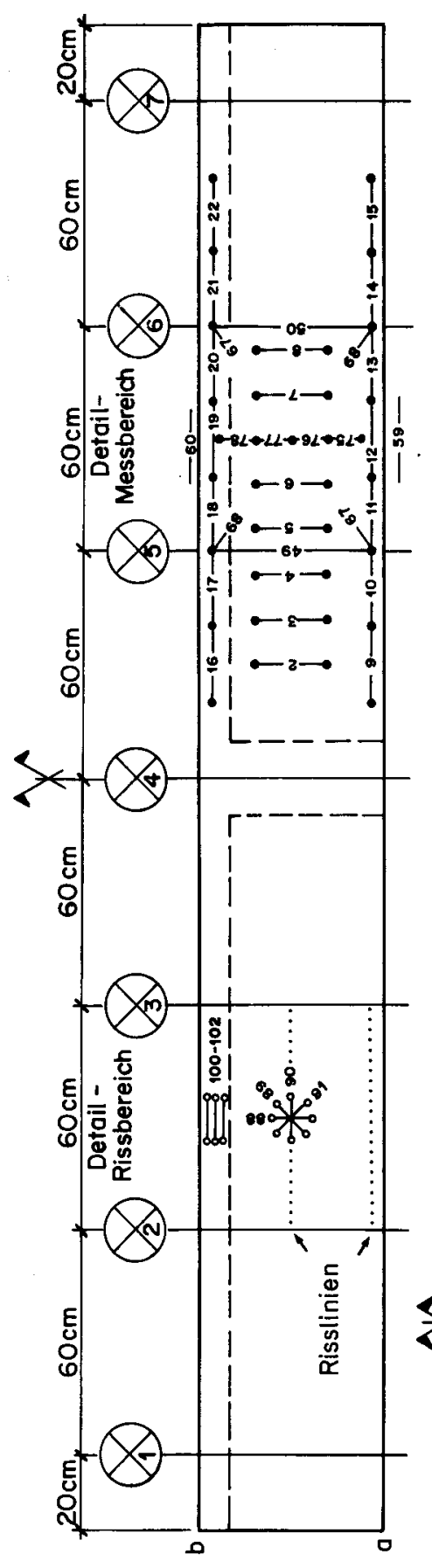

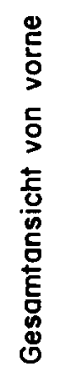
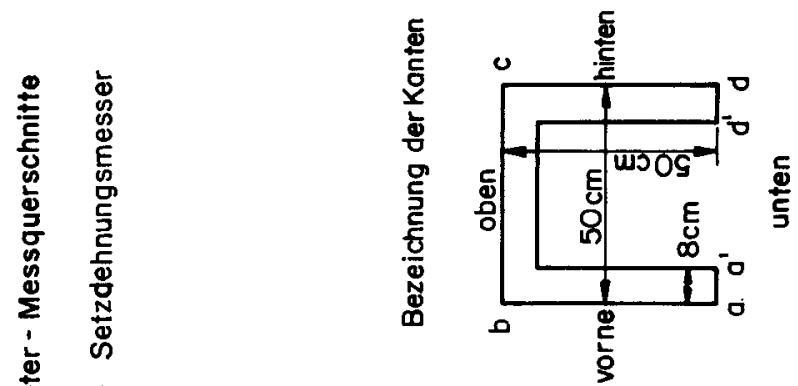

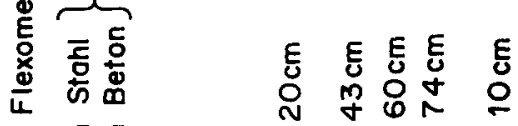

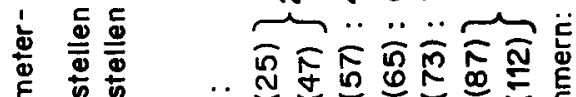

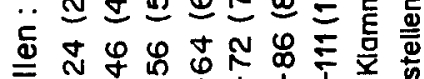

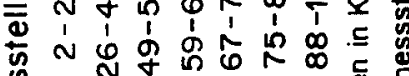

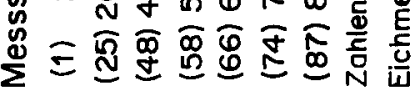
$\otimes 1 !$

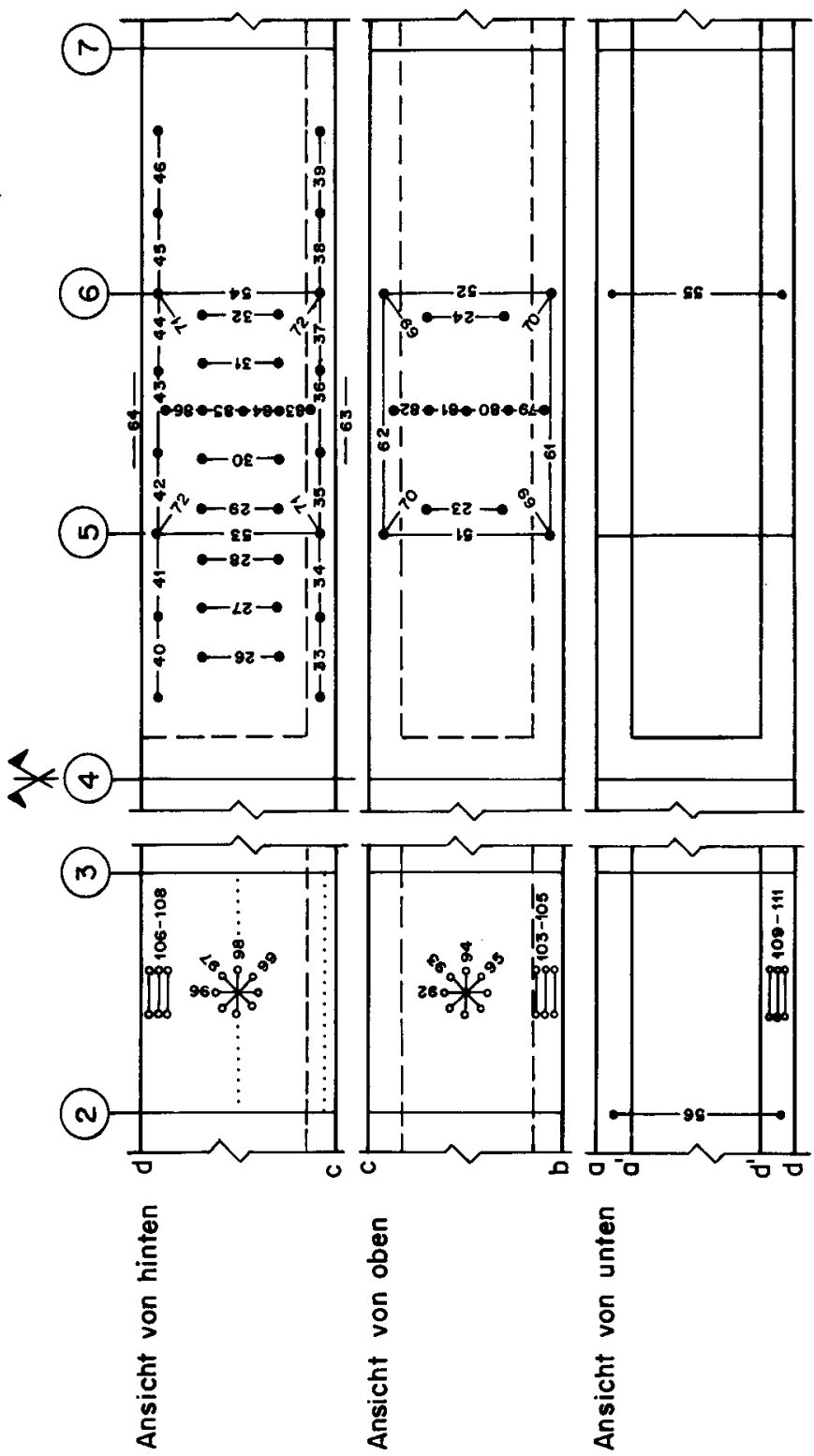

$\sum_{i}^{n}$

高

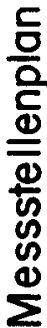

$\ddot{\sigma}$

믐 


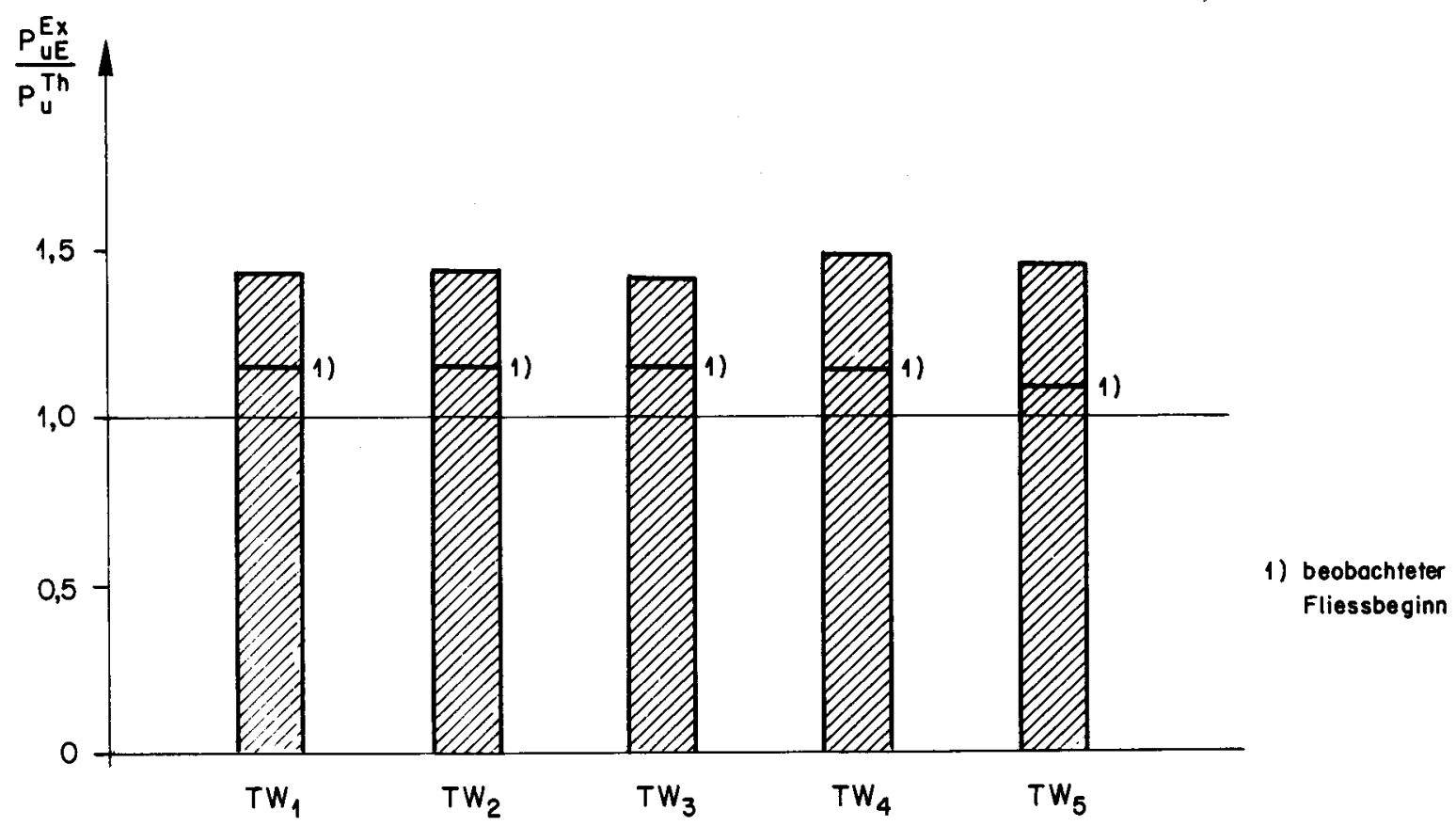

Bild 20: Vergleich der gemessenen Bruchlasten mit den theoretischen Bruchlasten des Scheibenmodells
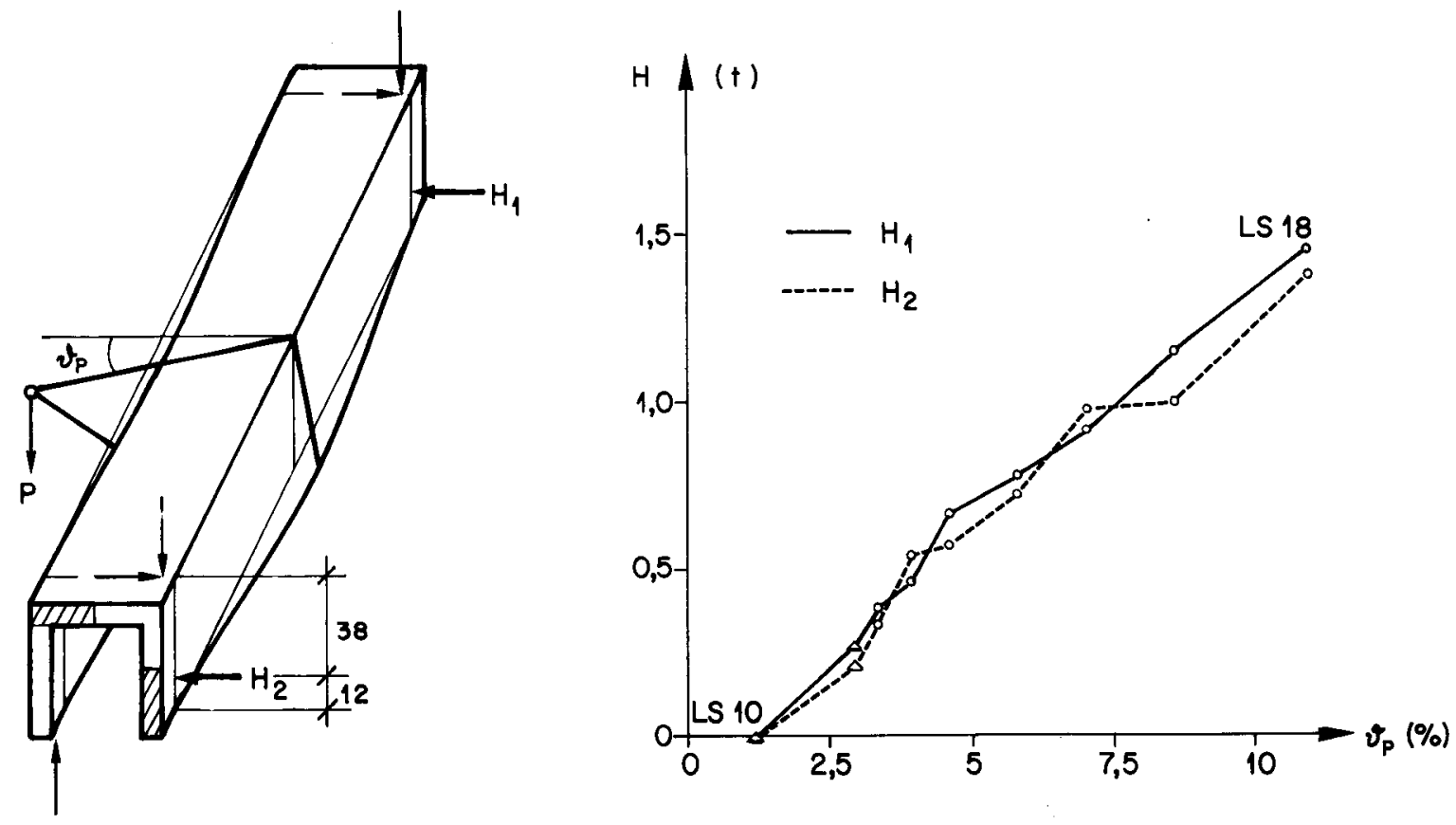

Bild 21: Haltekräfte der hinteren Platte von $T_{1}$ in Funktion der Lastarm - Verdrehung $v_{p}$ 


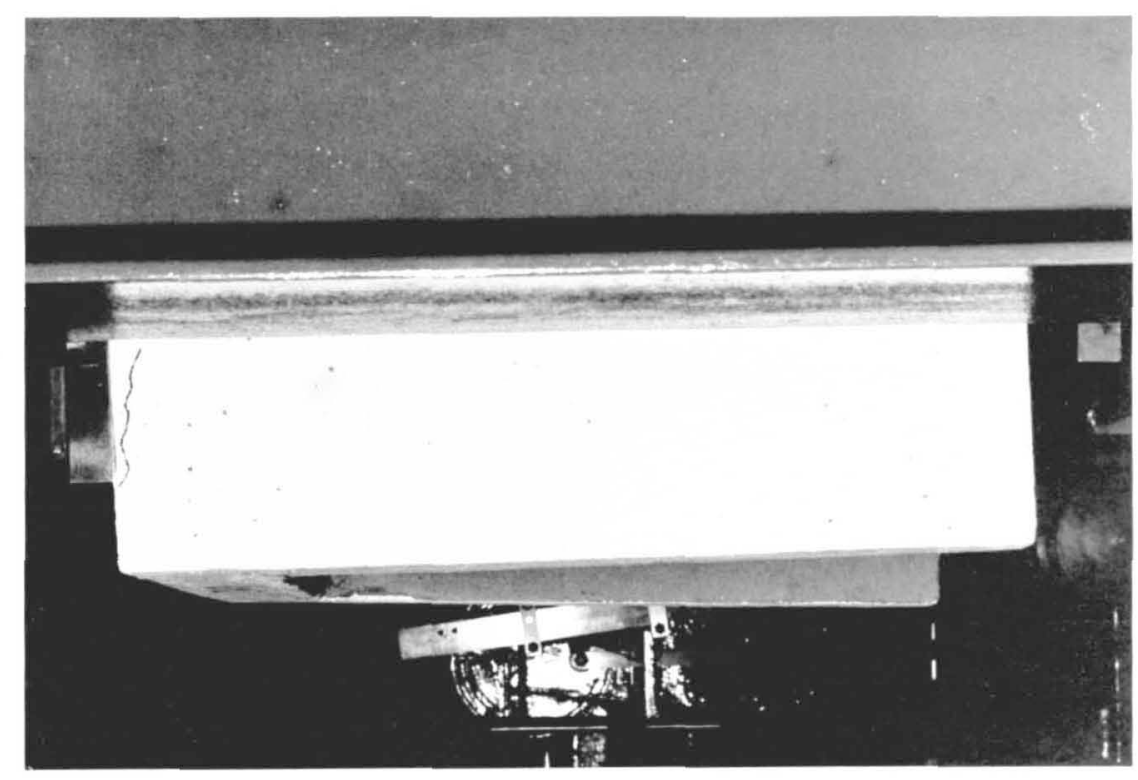

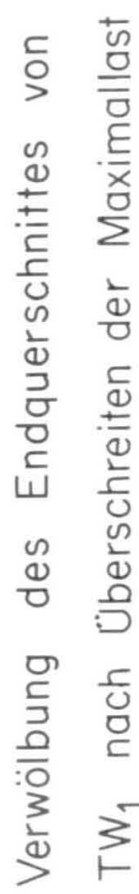

m

N

믐

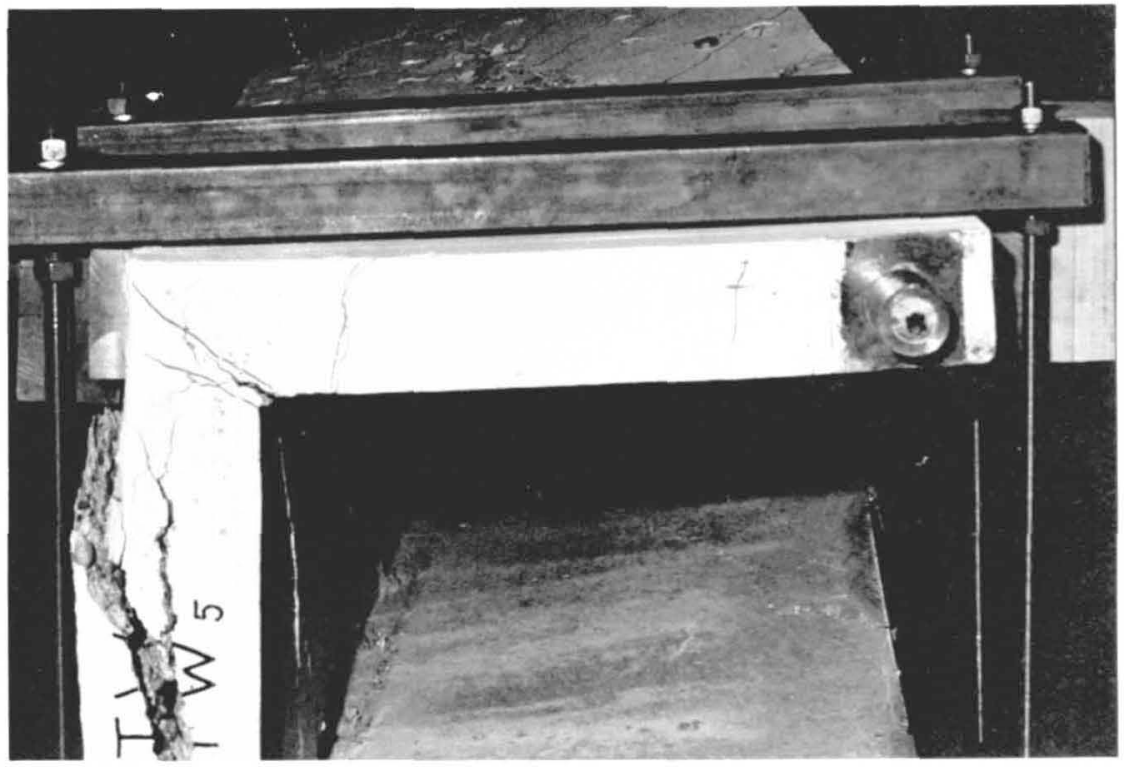

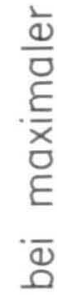

$3^{10}$

둥

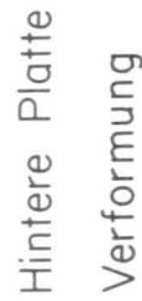

N

믐 


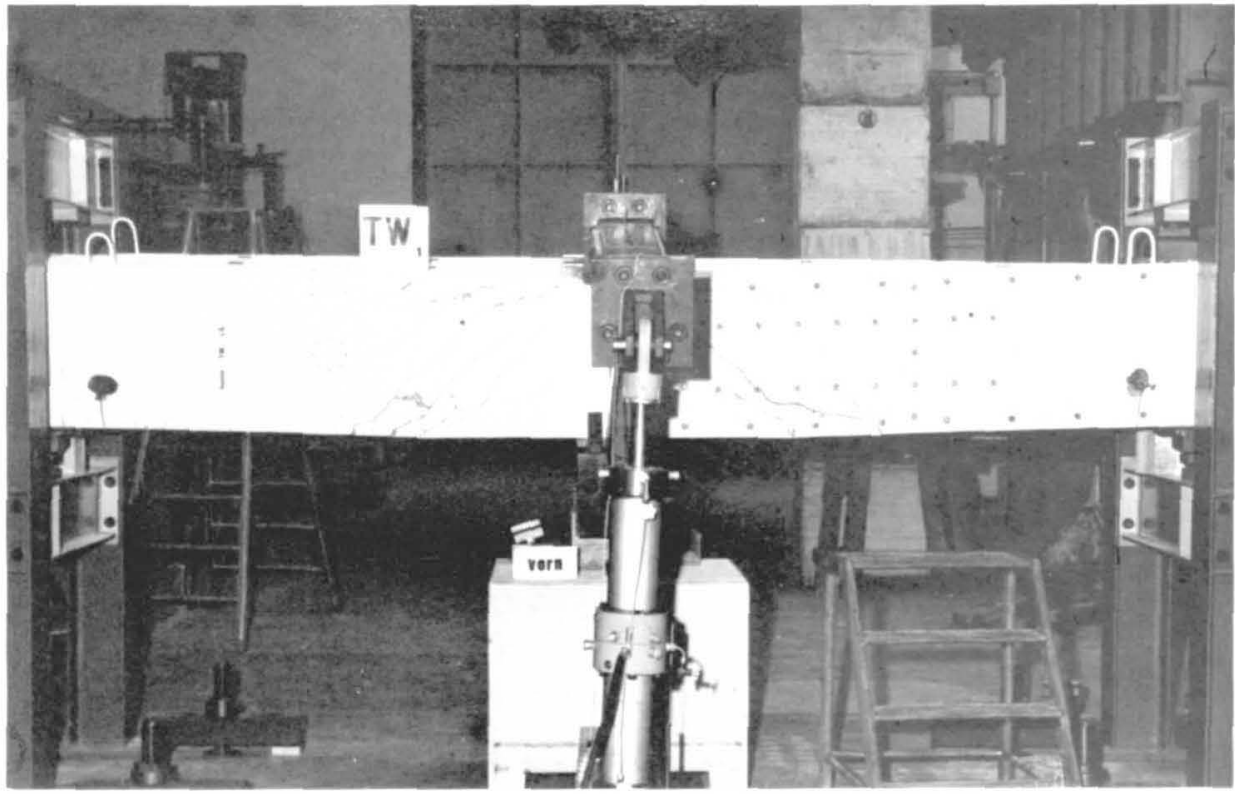

Bild 24: Balken TW 1 nach Überschreiten der Maximallast

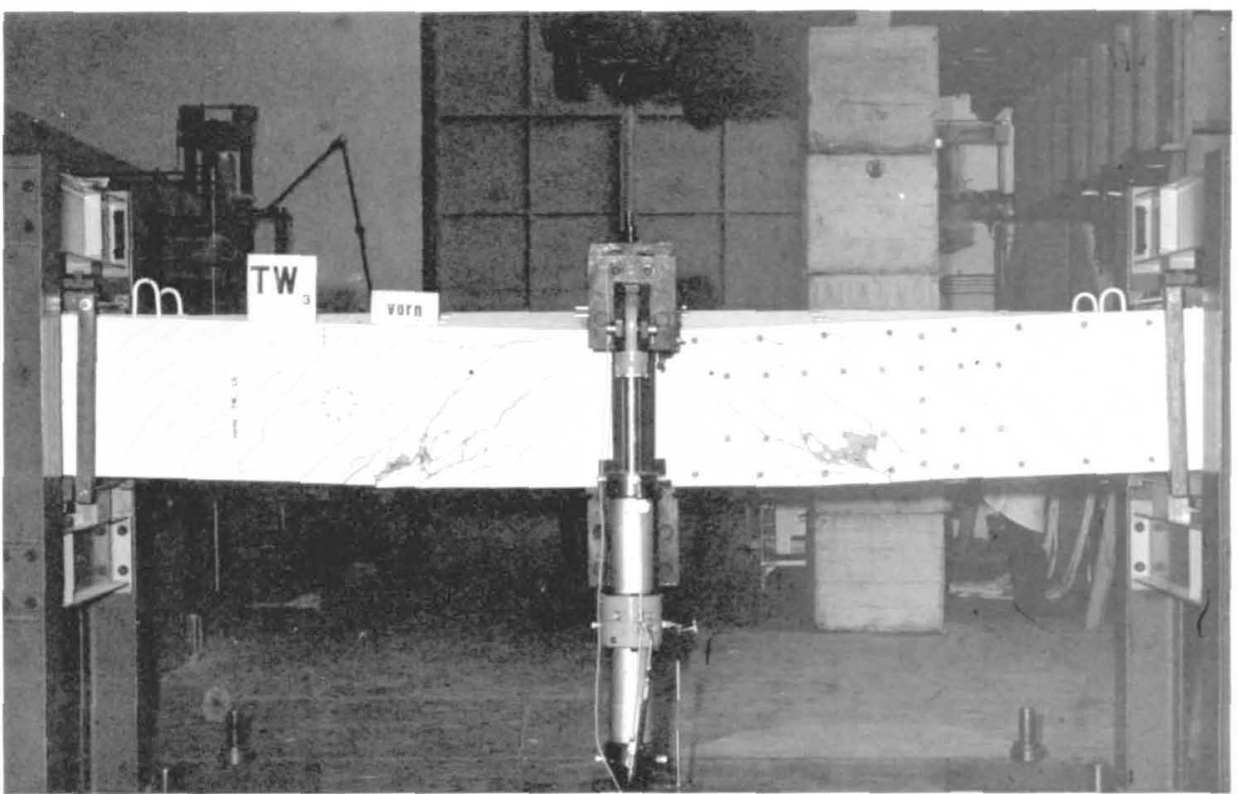

Bild 25: Balken $\mathrm{TW}_{3}$ nach Überschreiten der Maximallast 

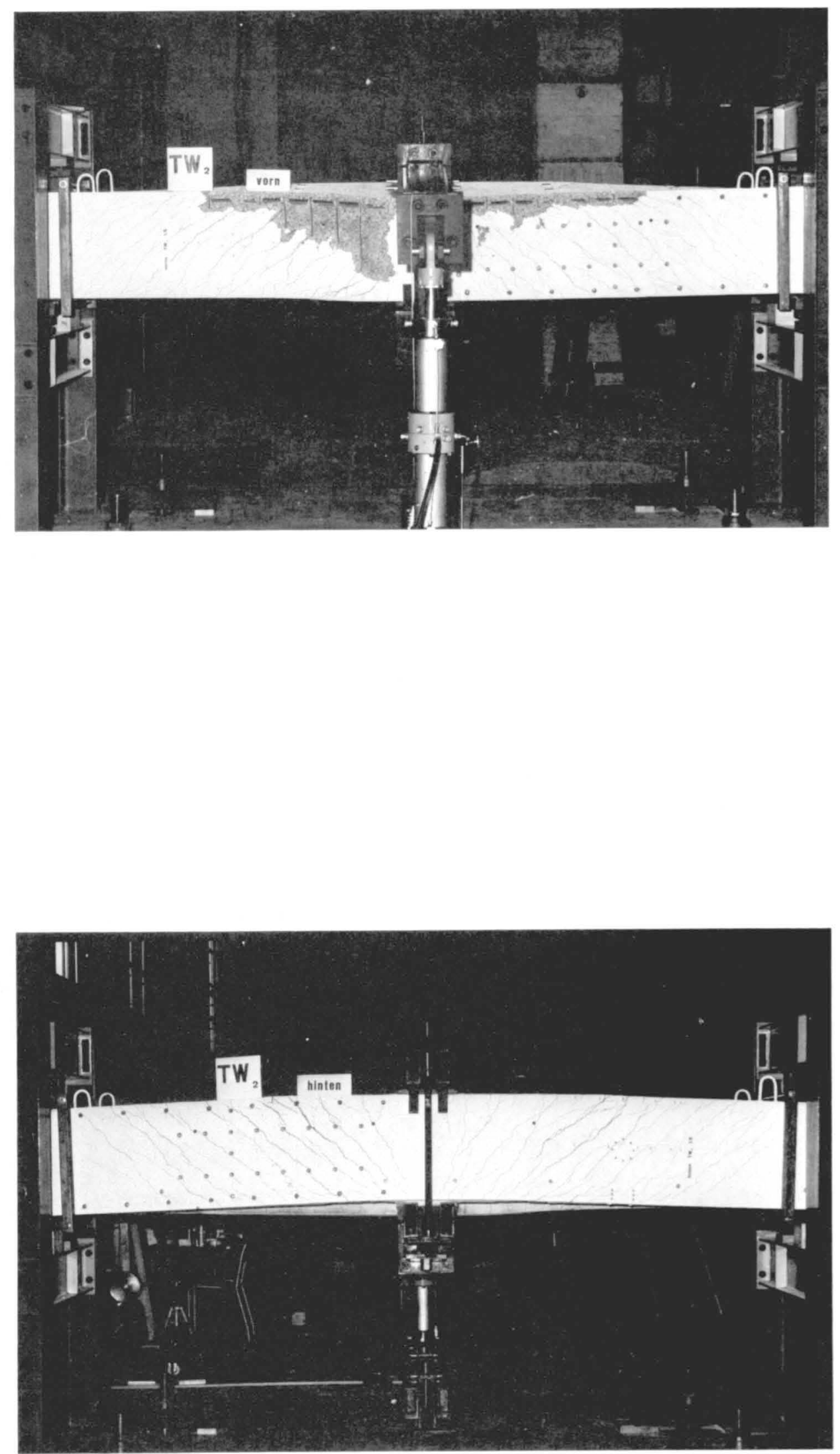

Bild 26: Balken $\mathrm{TW}_{2}$ nach Überschreiten der Maximallast 


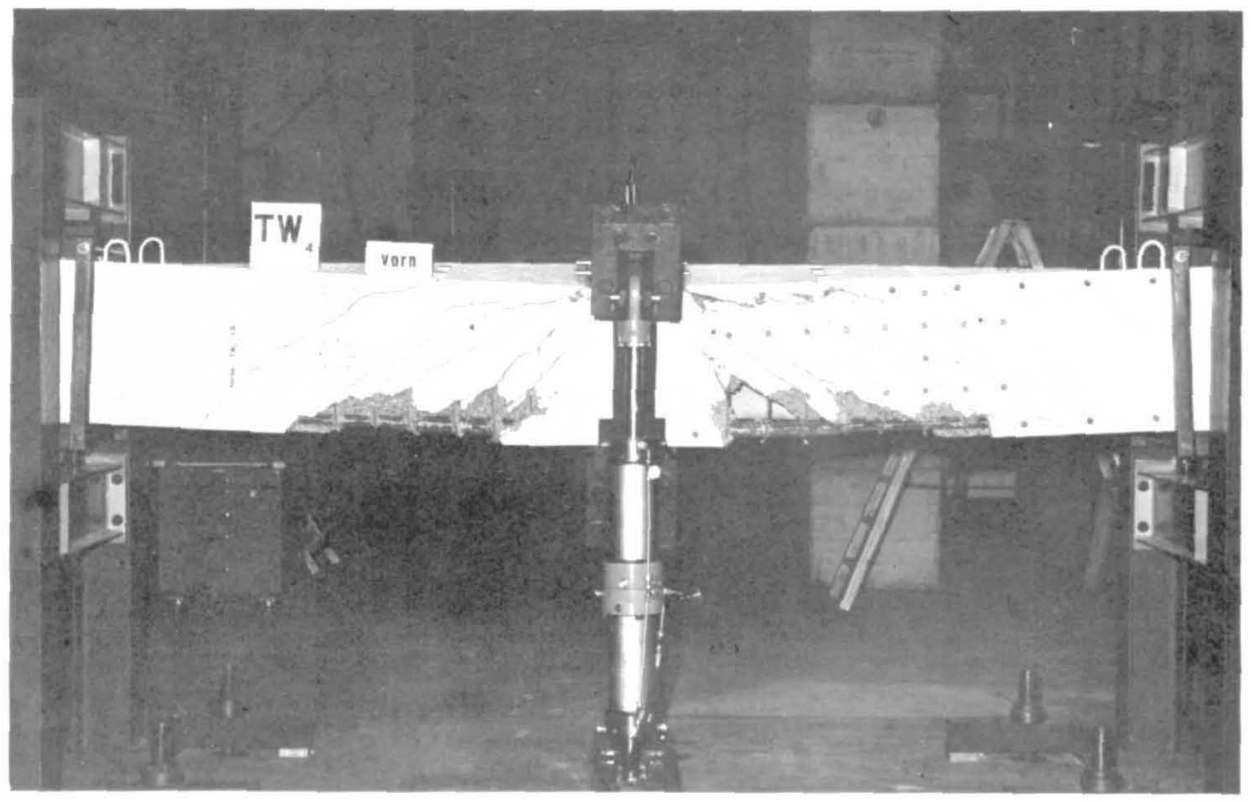

Bild 27: Balken $\mathrm{TW}_{4}$ nach Überschreiten der Maximallast

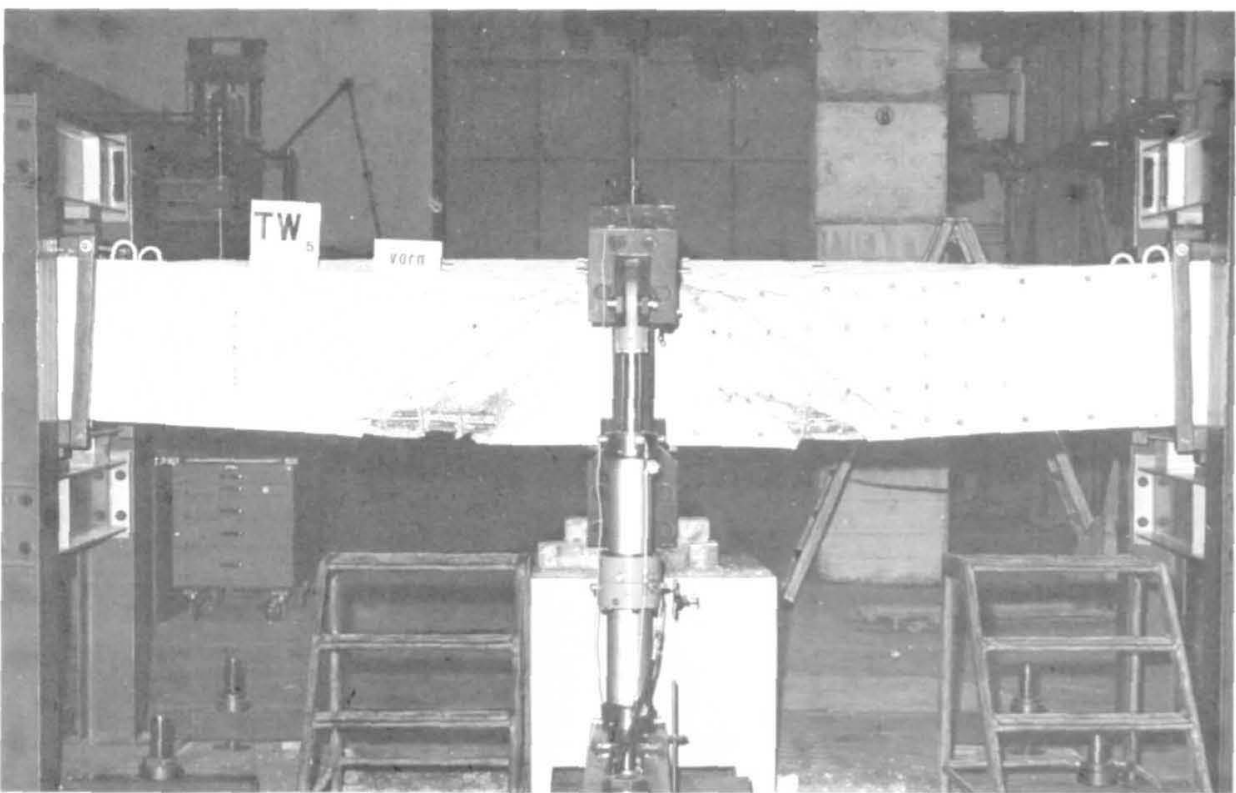

Bild 28: Balken TW $_{5}$ nach Überschreiten der Maximallast 


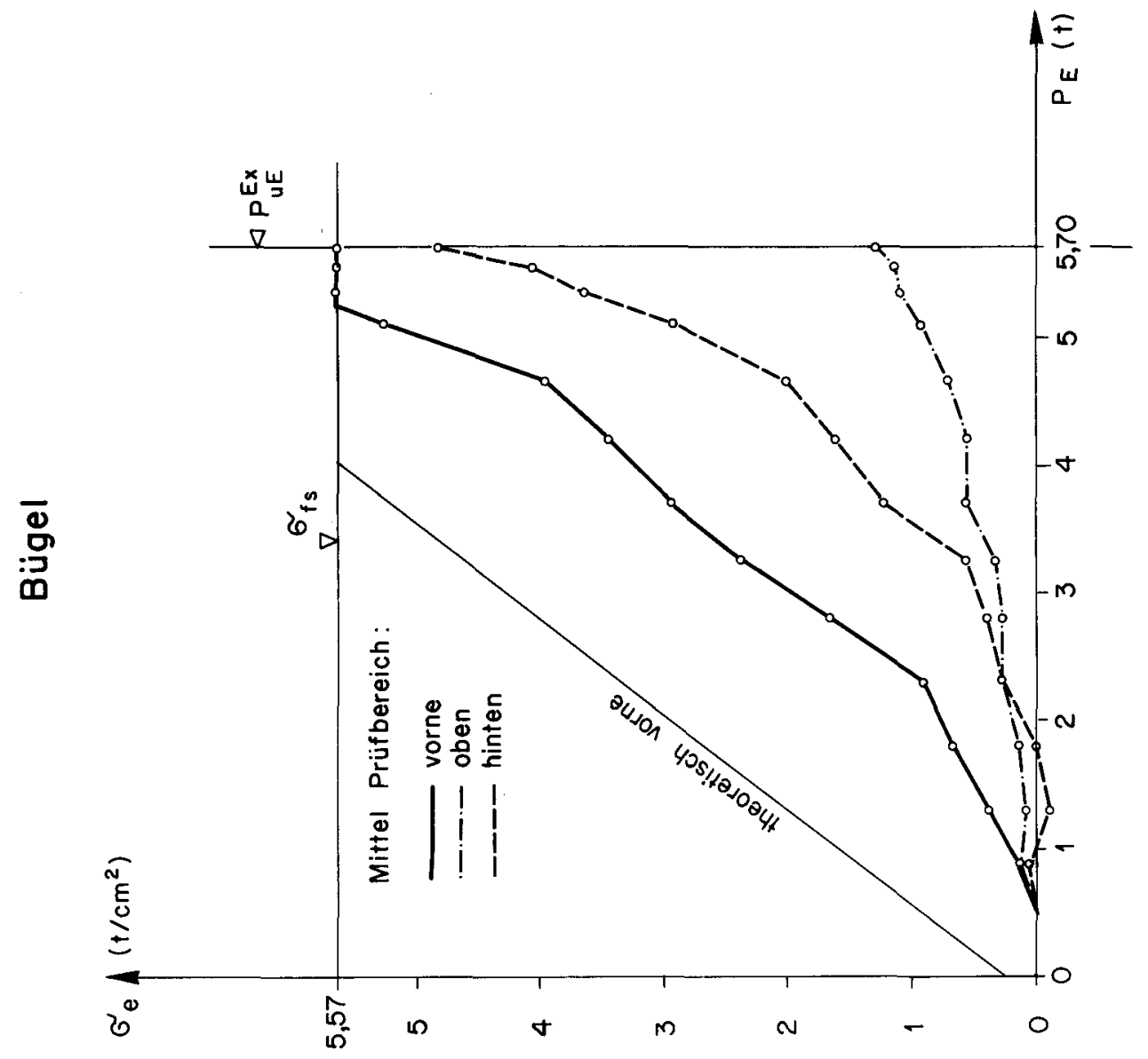

号

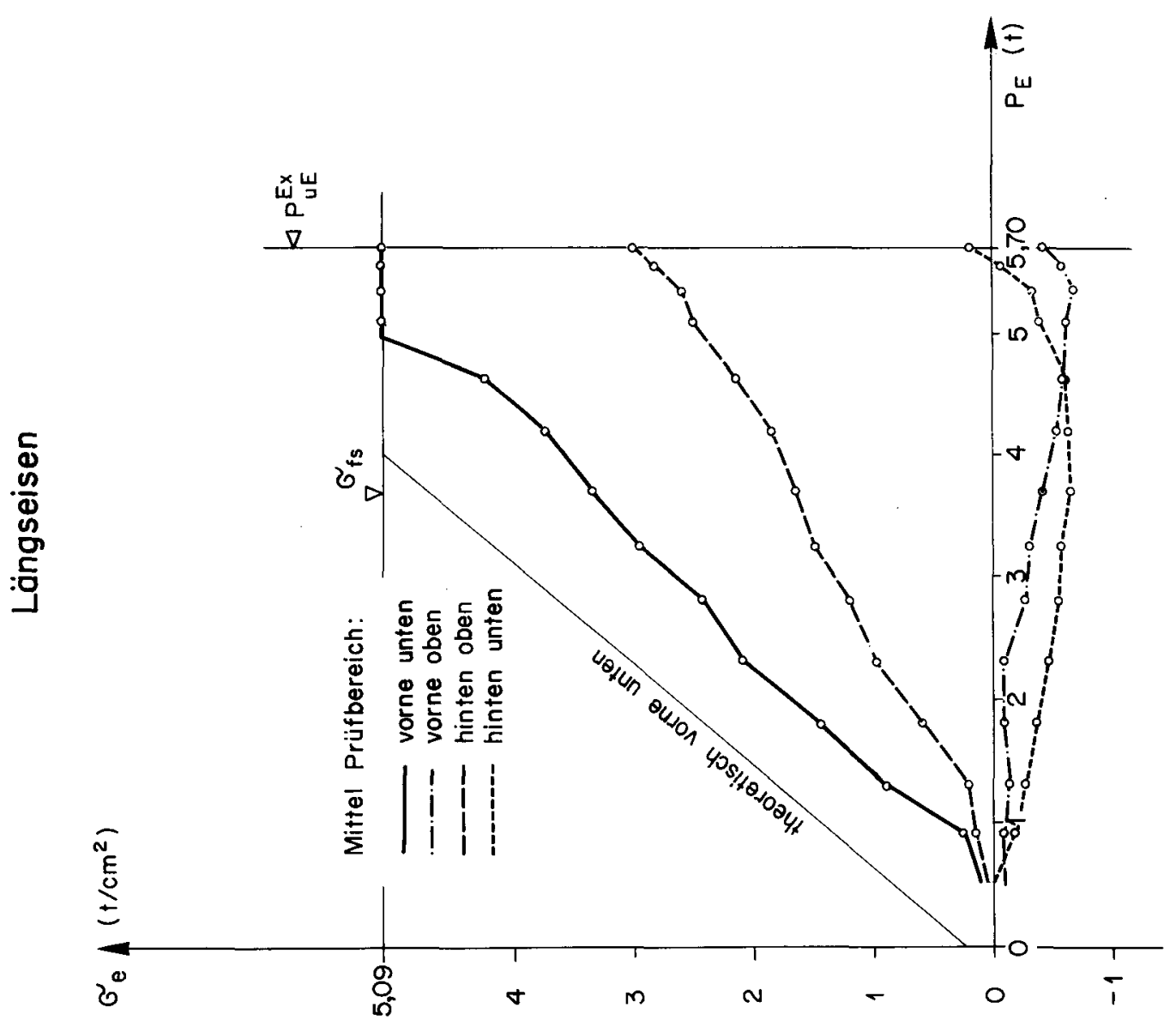

은 . 


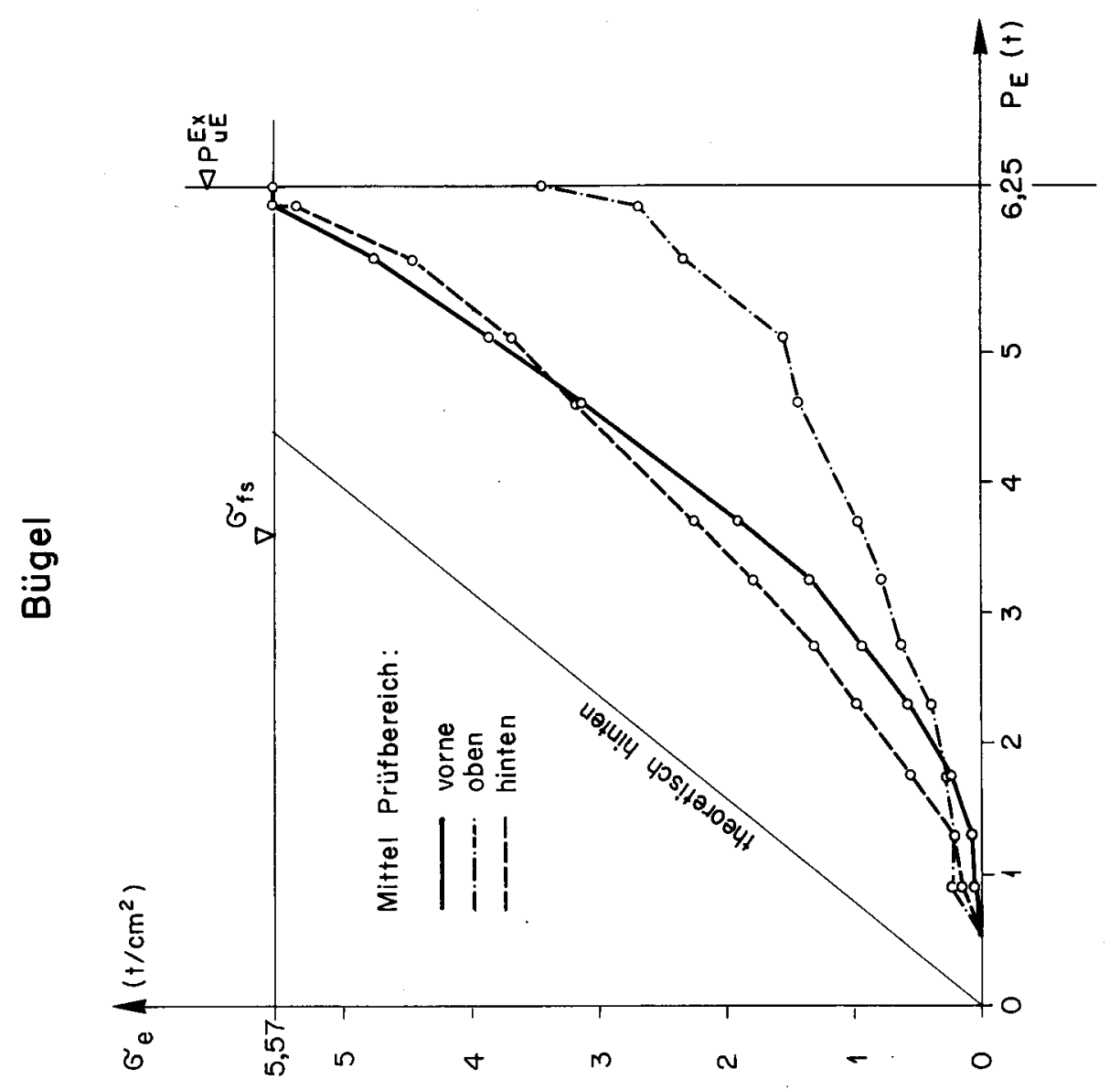

ㅁ
$\frac{5}{5}$
$\frac{5}{0}$
๓
$\frac{1}{0}$

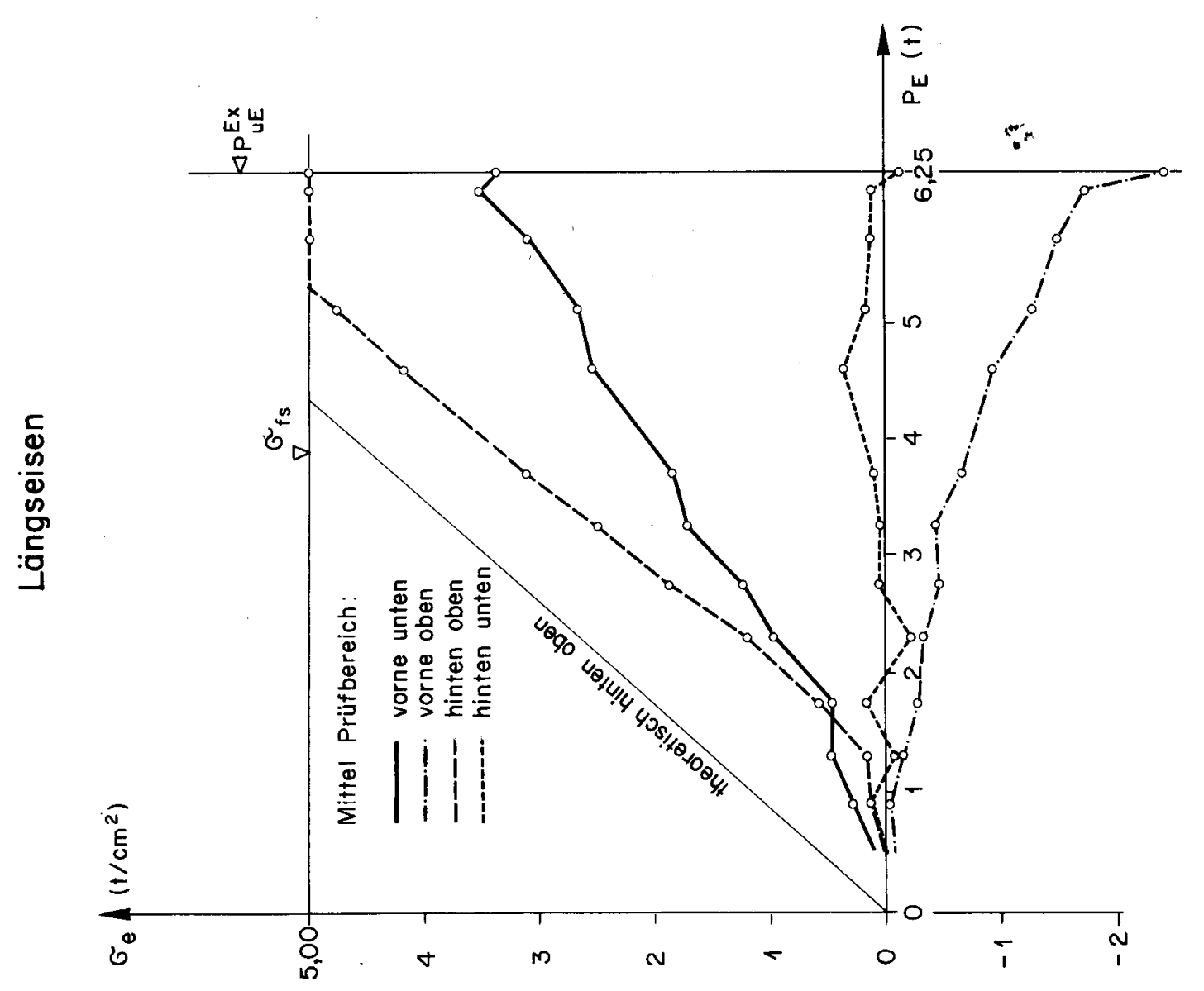

흘 

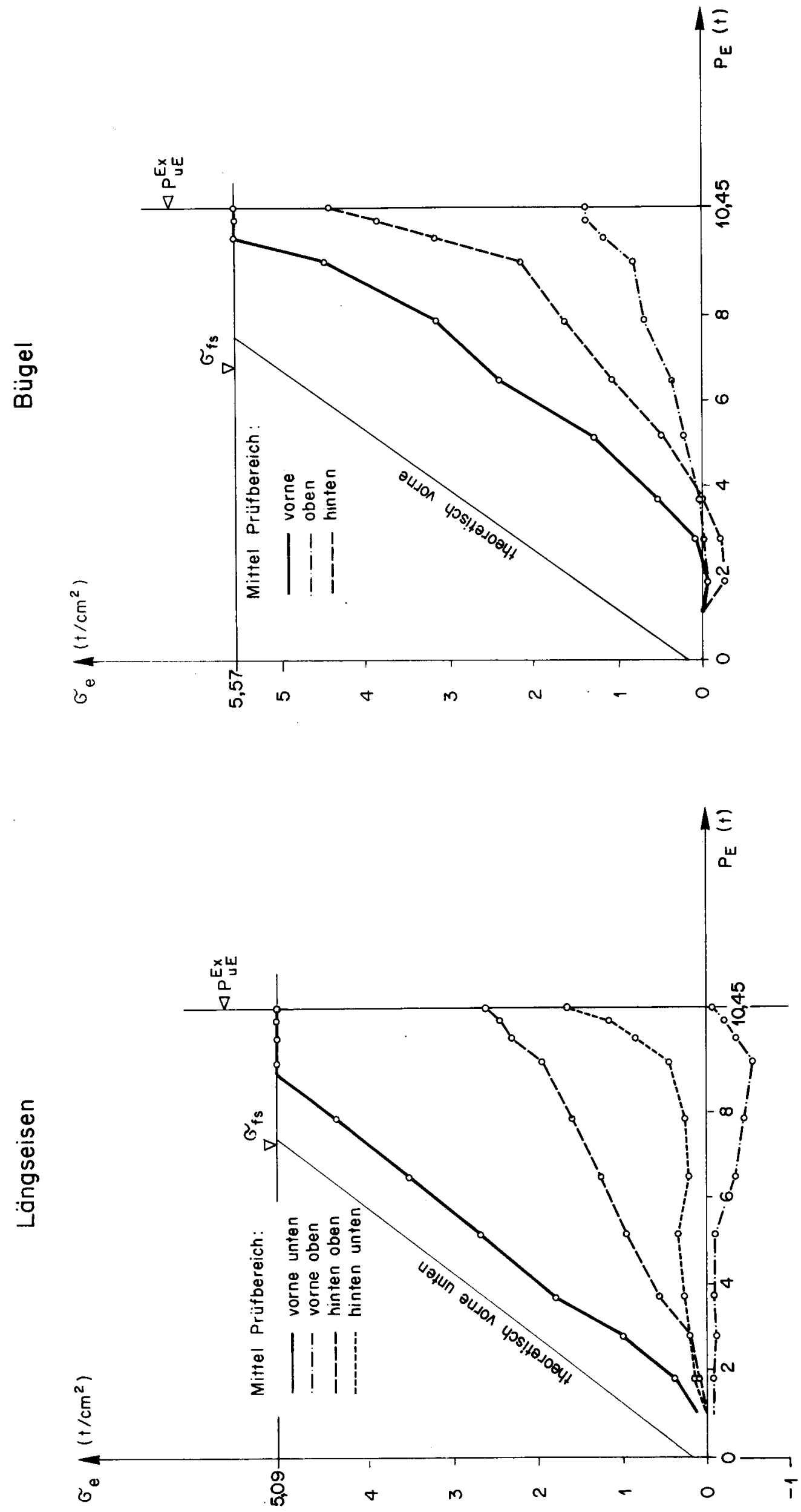

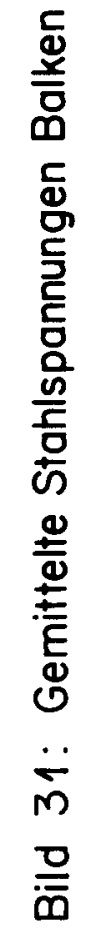




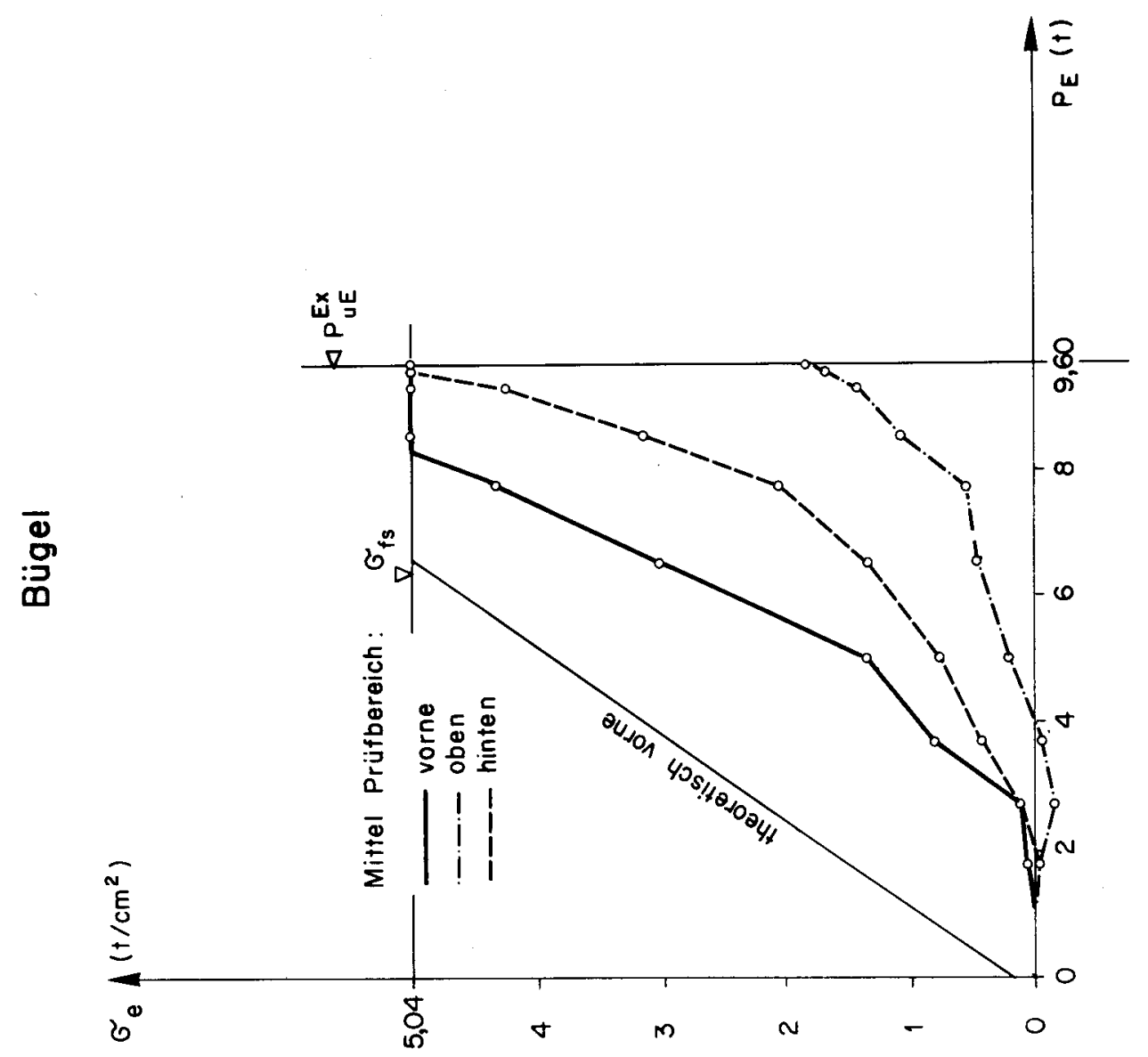

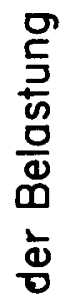

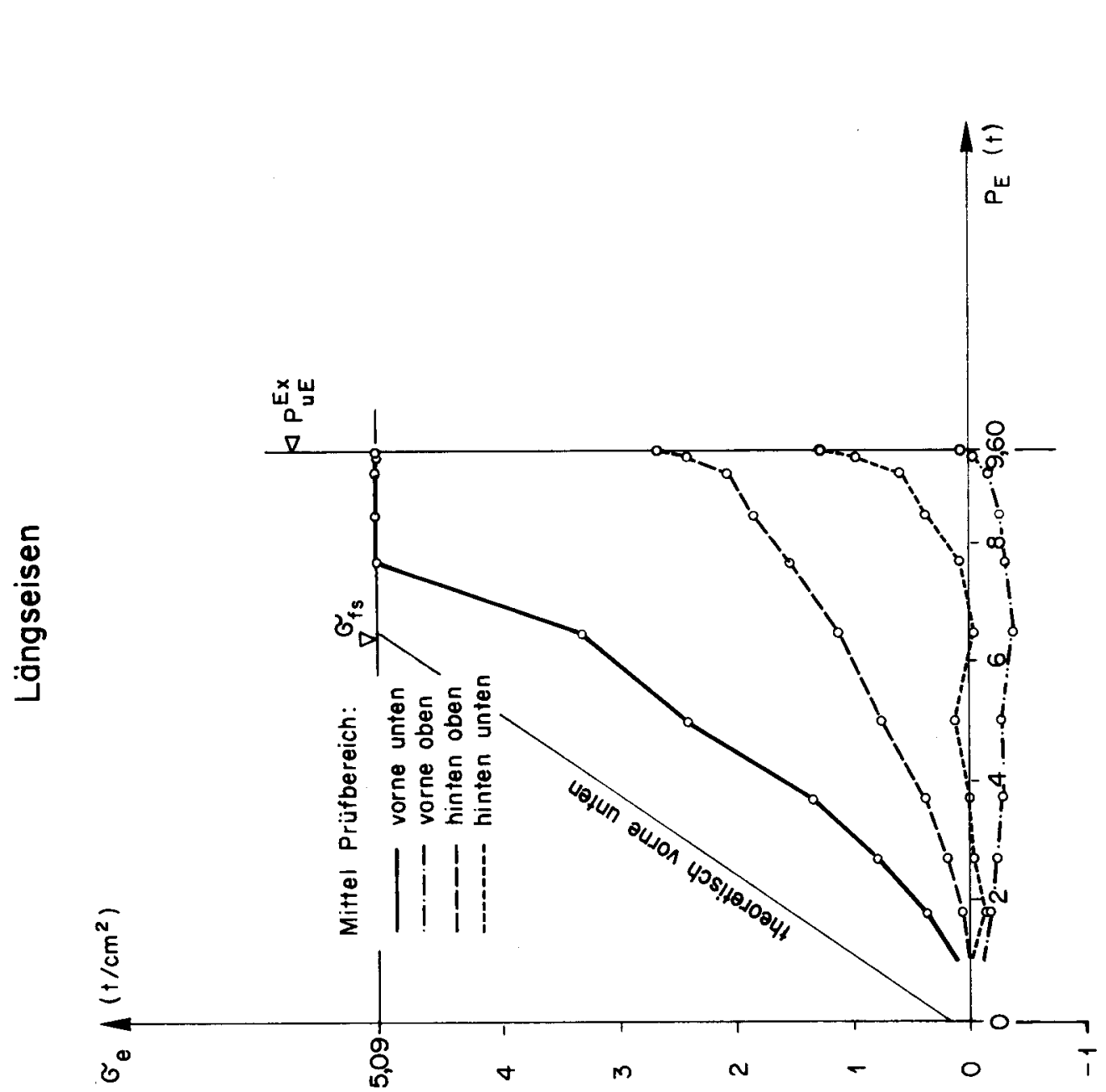

亭

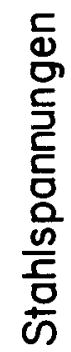

पू

in

음 


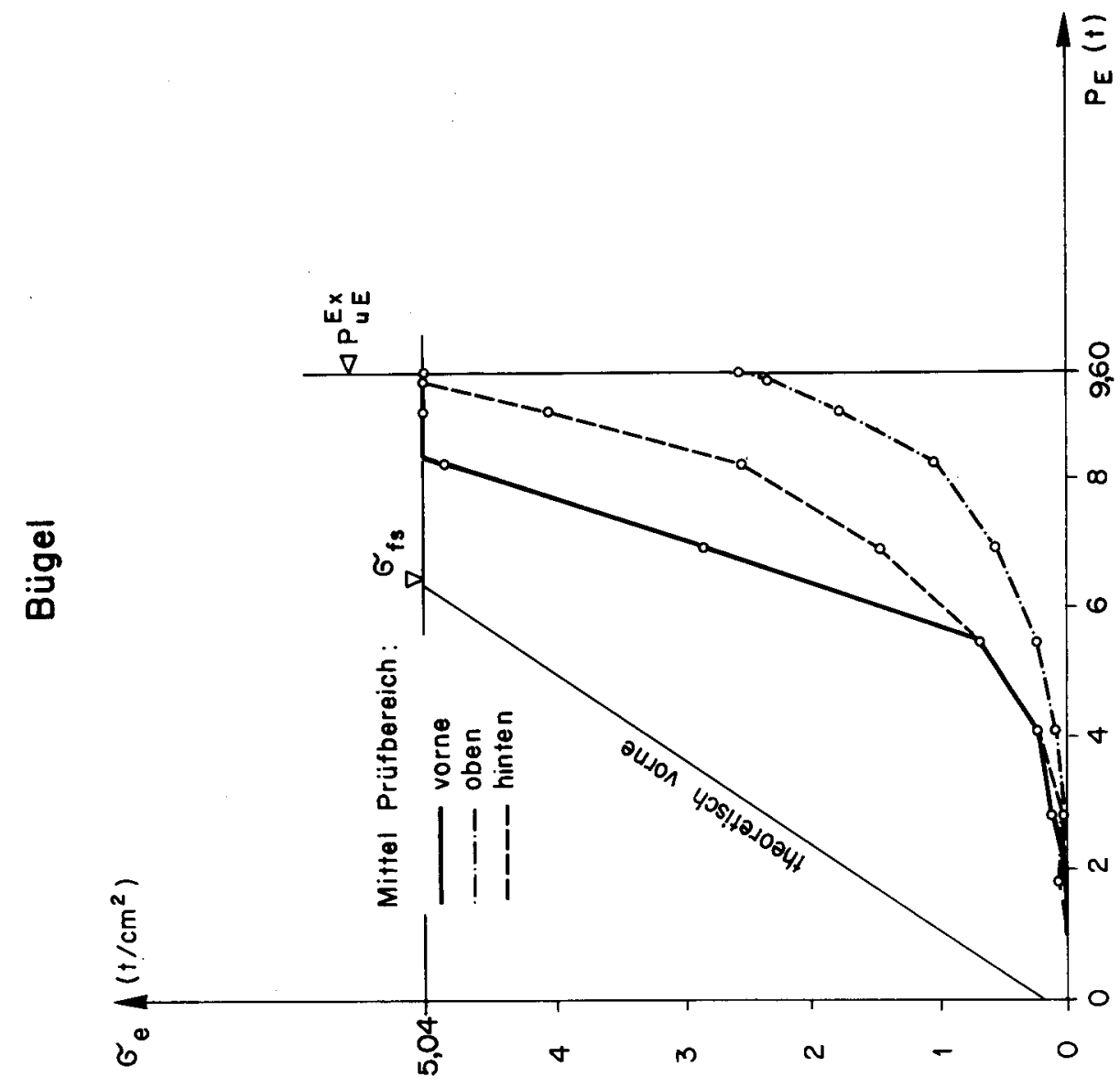

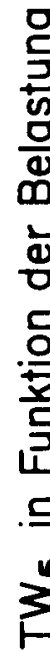

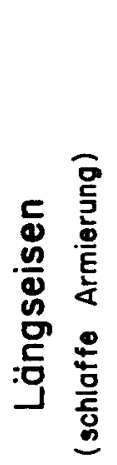

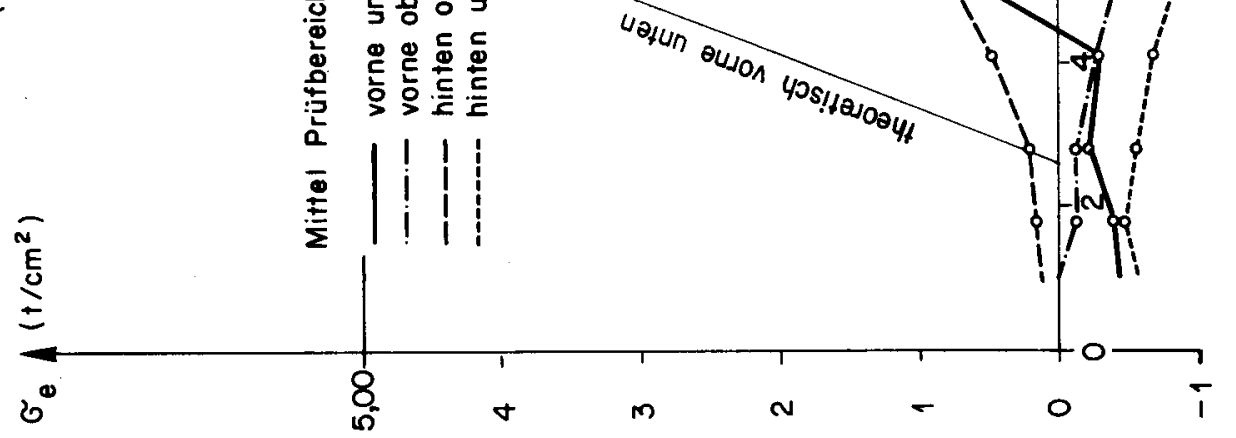

돟 

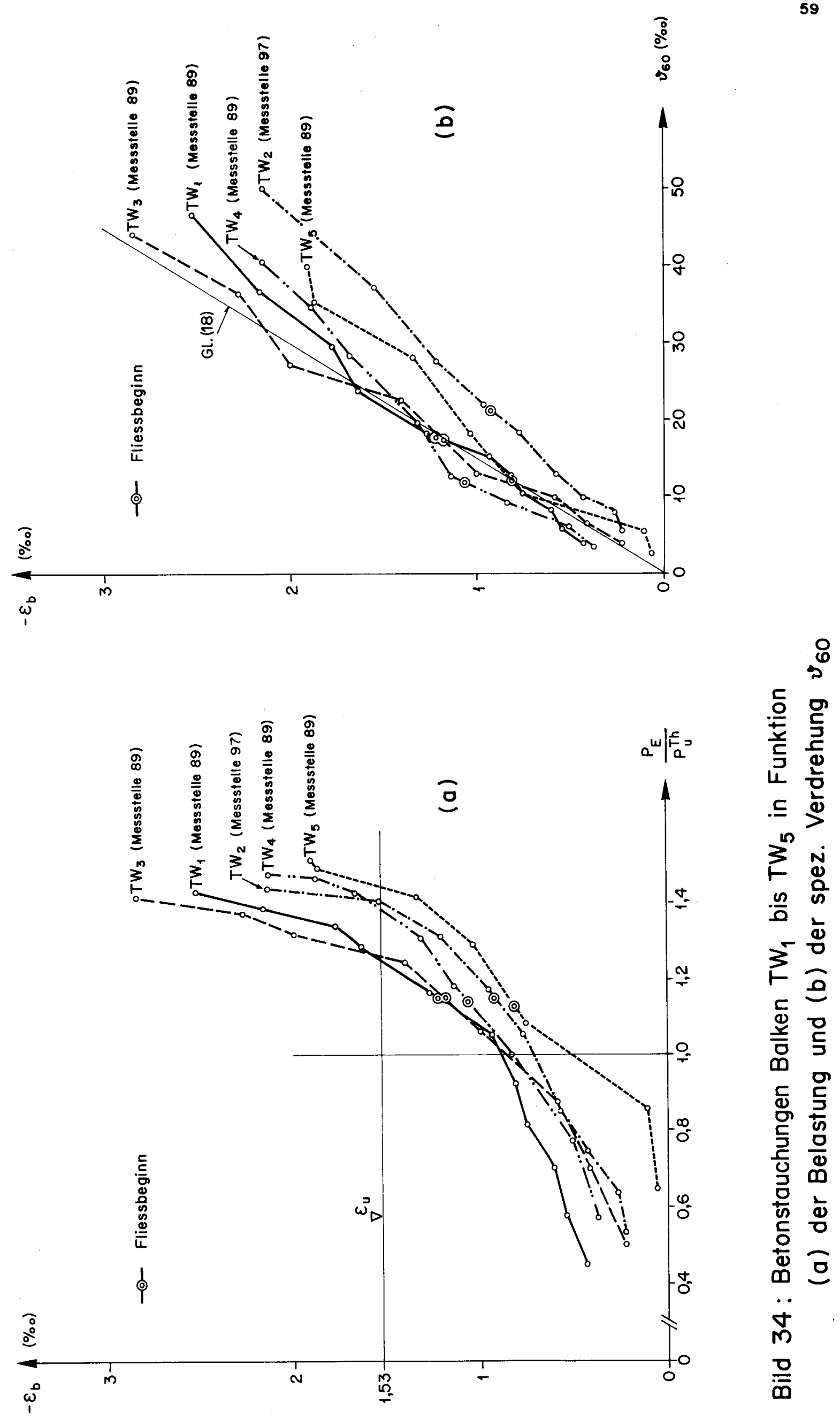


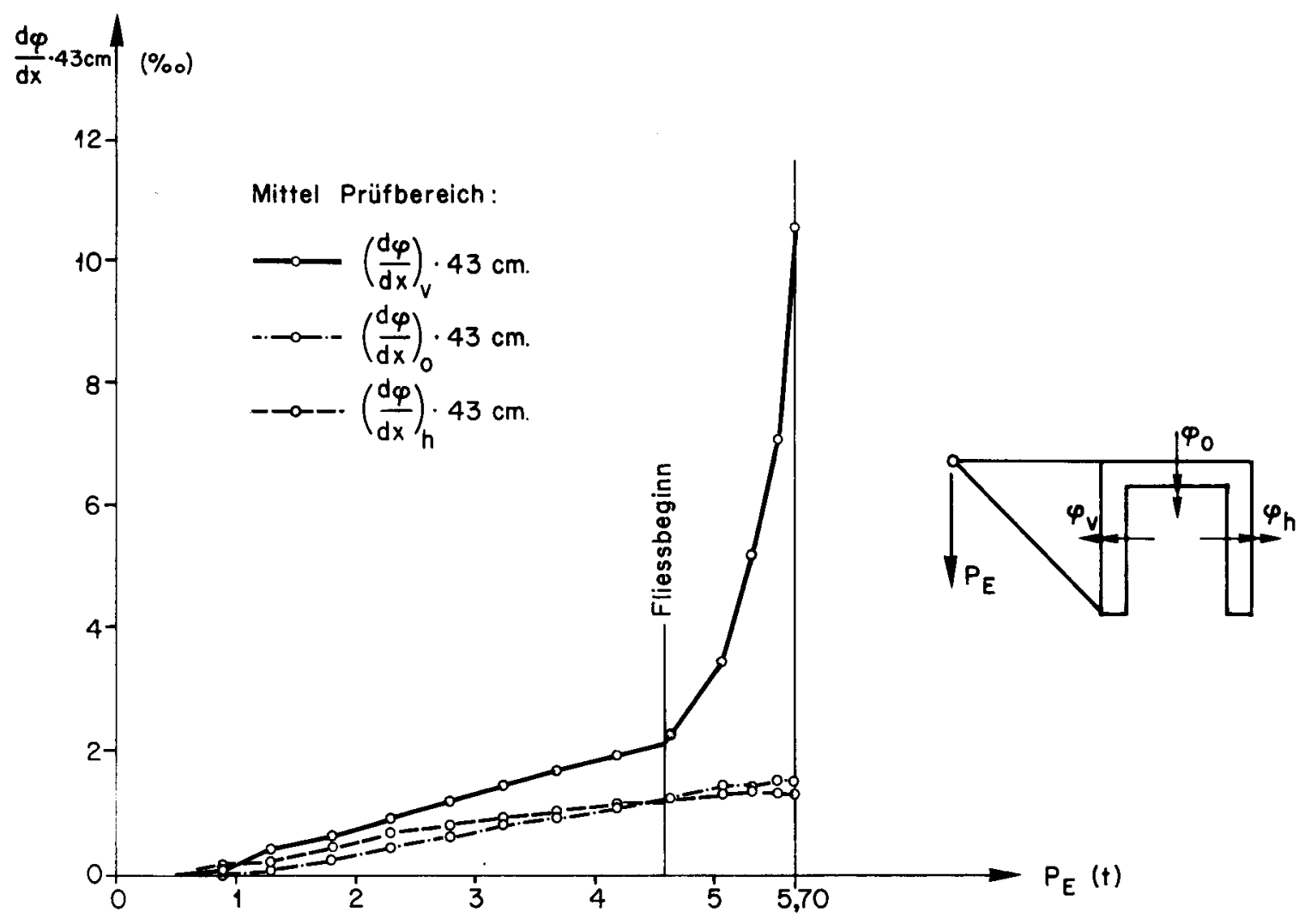

Bild 35: Krümmungen $\frac{d \varphi}{d x}$ Balken $T W_{1}$ in Funktion der Belastung

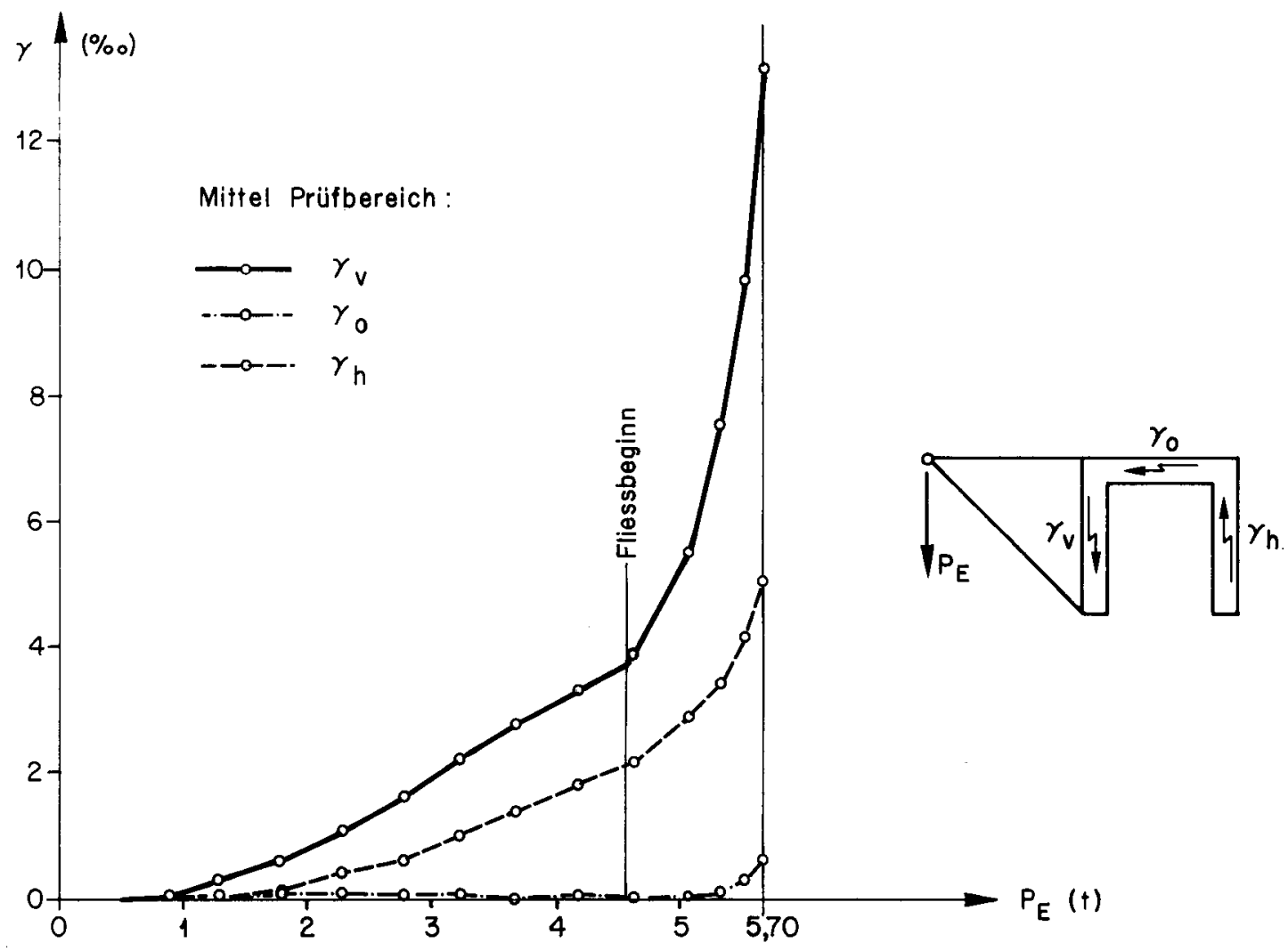

Bild 36: Schiebungen $\gamma$ Balken $T W_{1}$ in Funktion der Belastung 


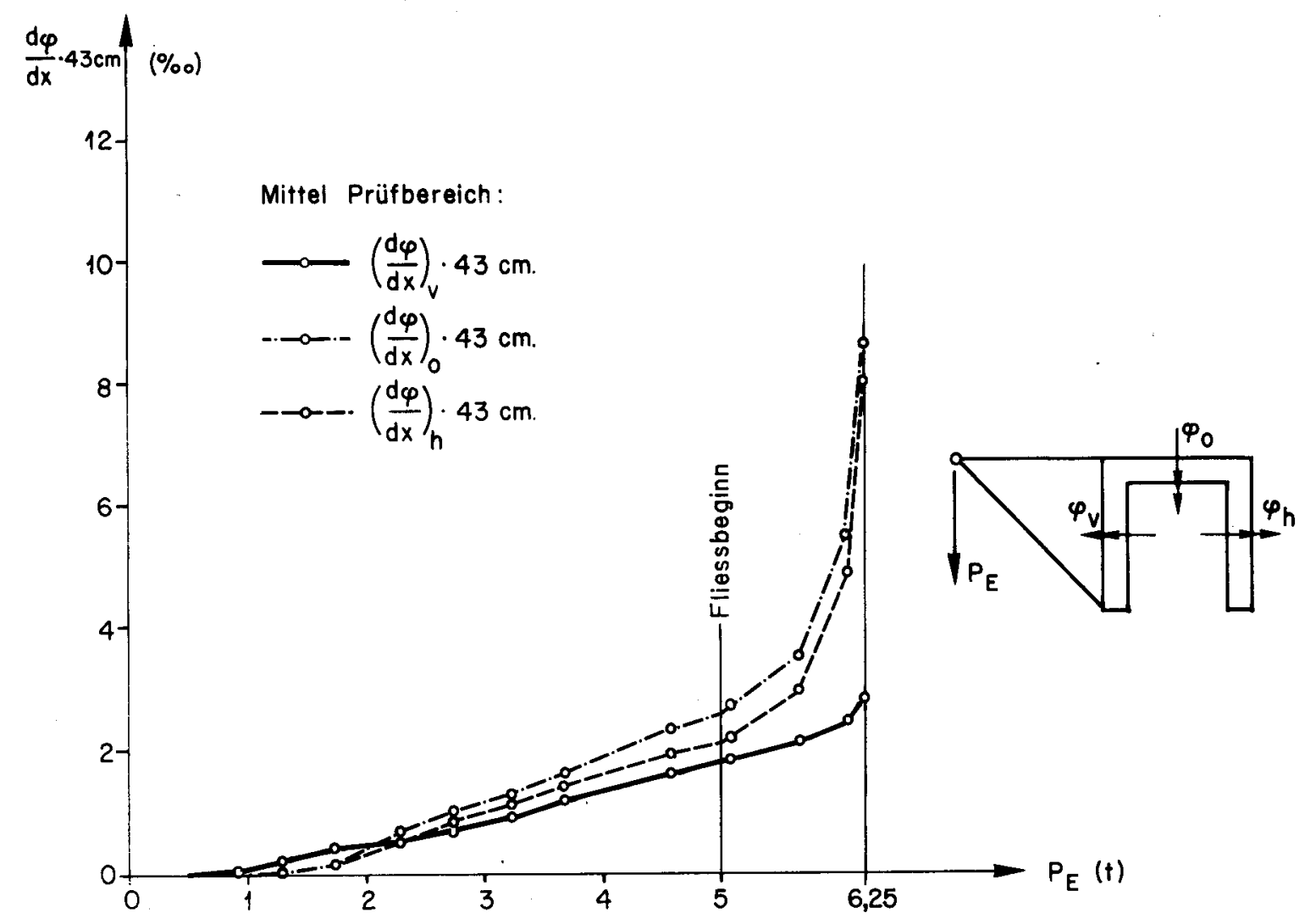

Bild 37: Krümmungen $\frac{d \varphi}{d x}$ Balken $T_{2}$ in Funktion der Belastung

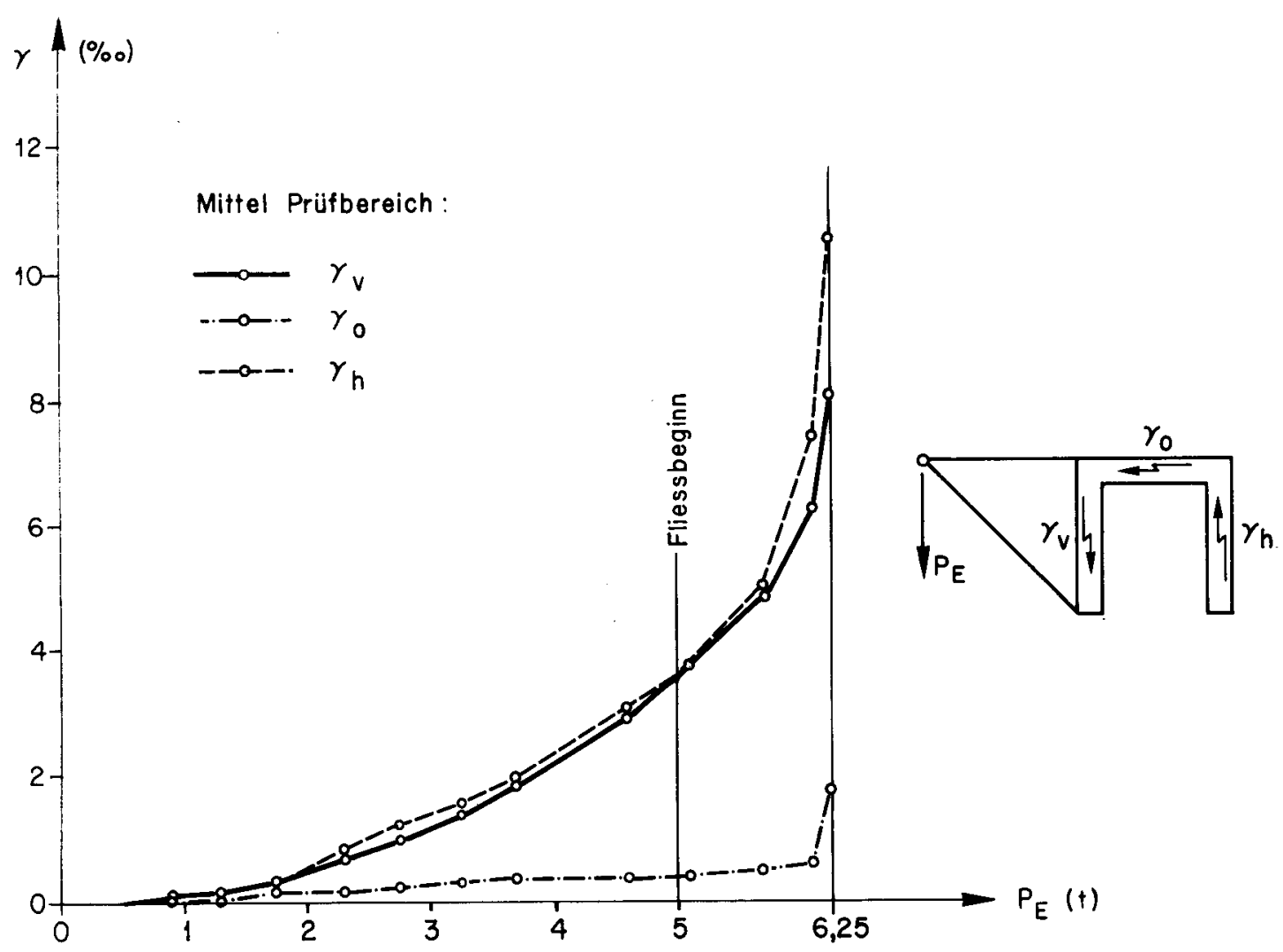

Bild 38: Schiebungen $\gamma$ Balken $\mathrm{TW}_{2}$ in Funktion der Belastung 


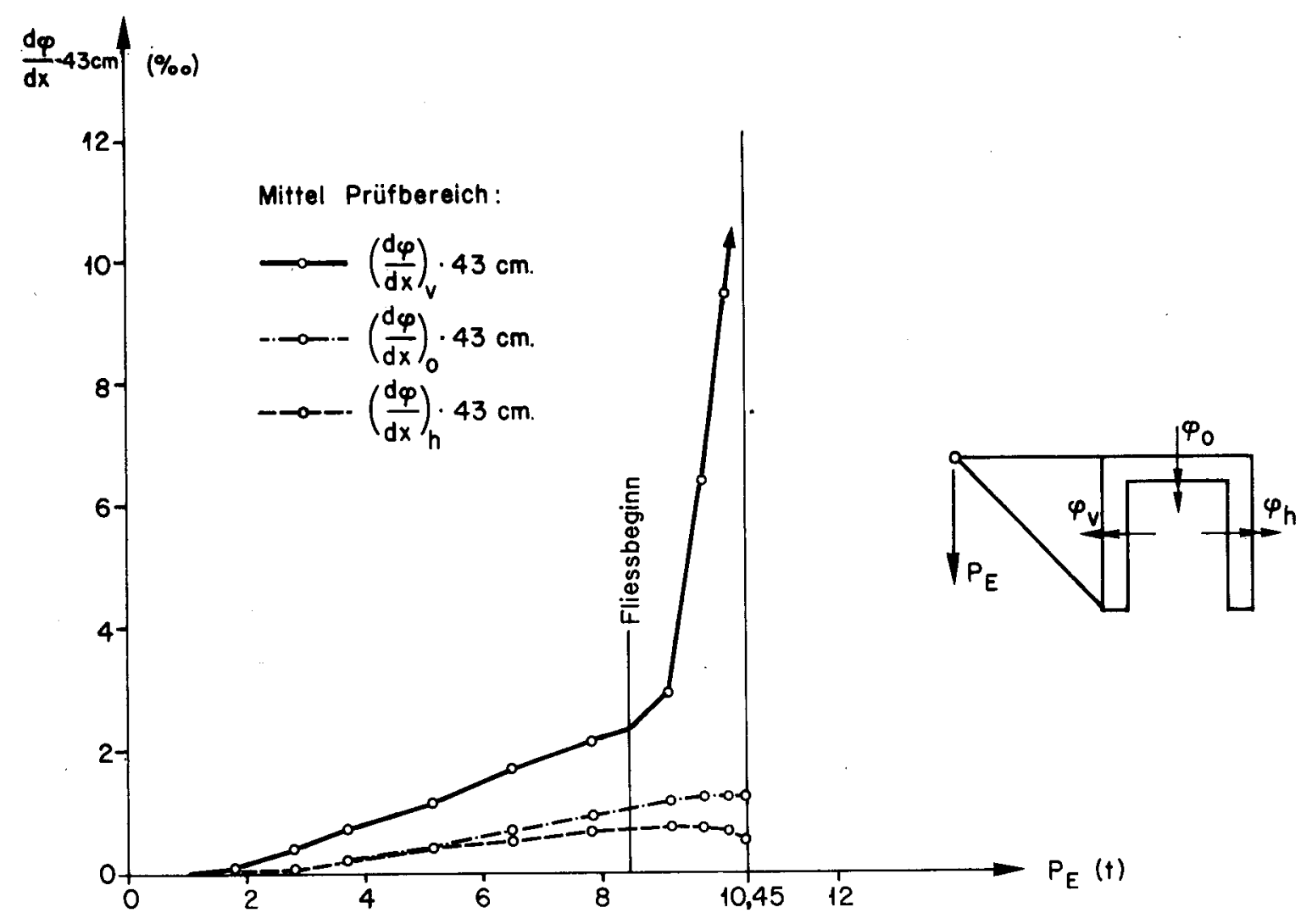

Bild 39: Krümmungen $\frac{d \varphi}{d x}$ Balken $\mathrm{TW}_{3}$ in Funktion der Belastung

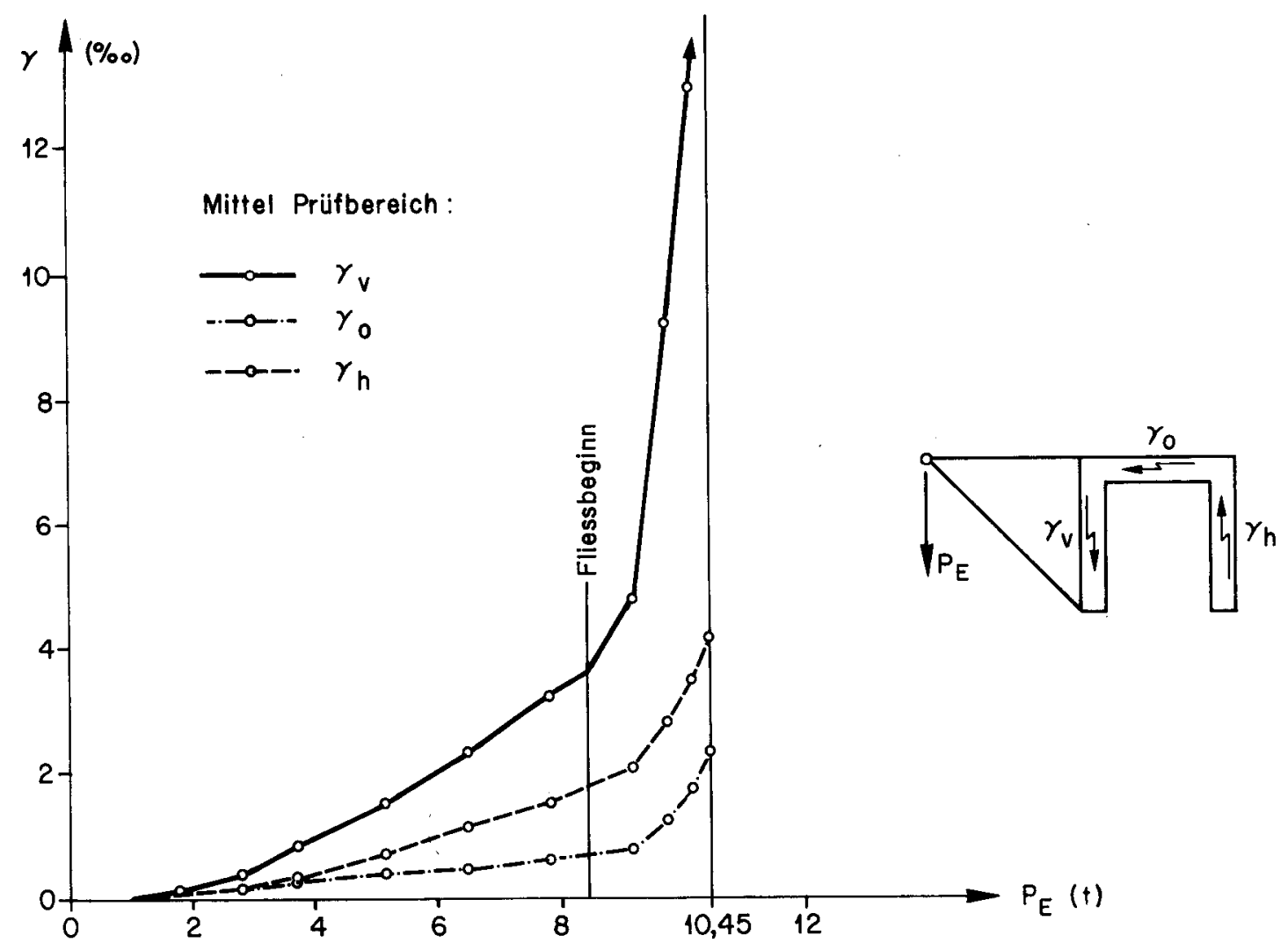

Bild 40: Schiebungen $y$ Balken $\mathrm{TW}_{3}$ in Funktion der Belastung 


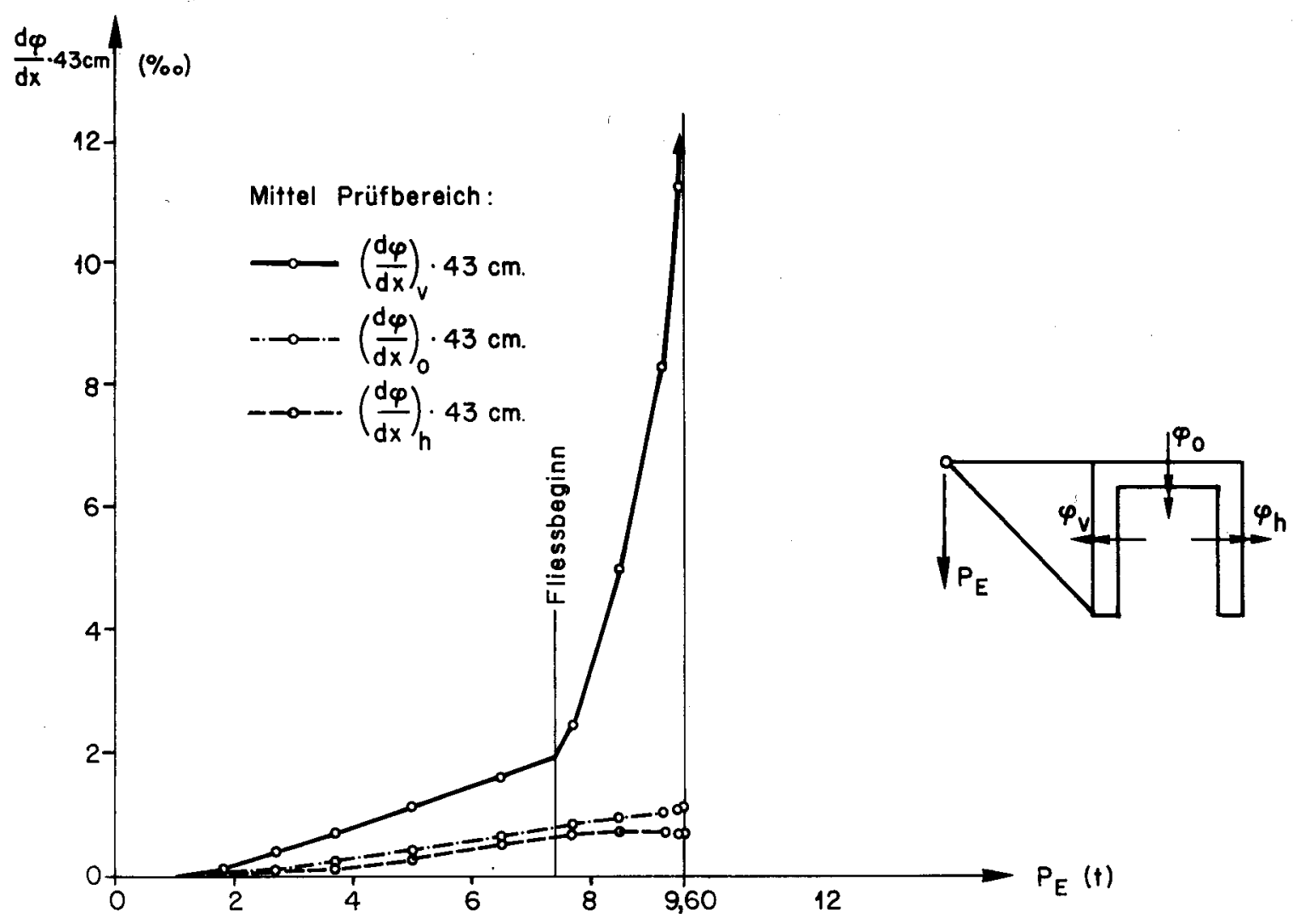

Bild 41 : Krümmungen $\frac{d \varphi}{d x}$ Balken $T W_{4}$ in Funktion der Belastung

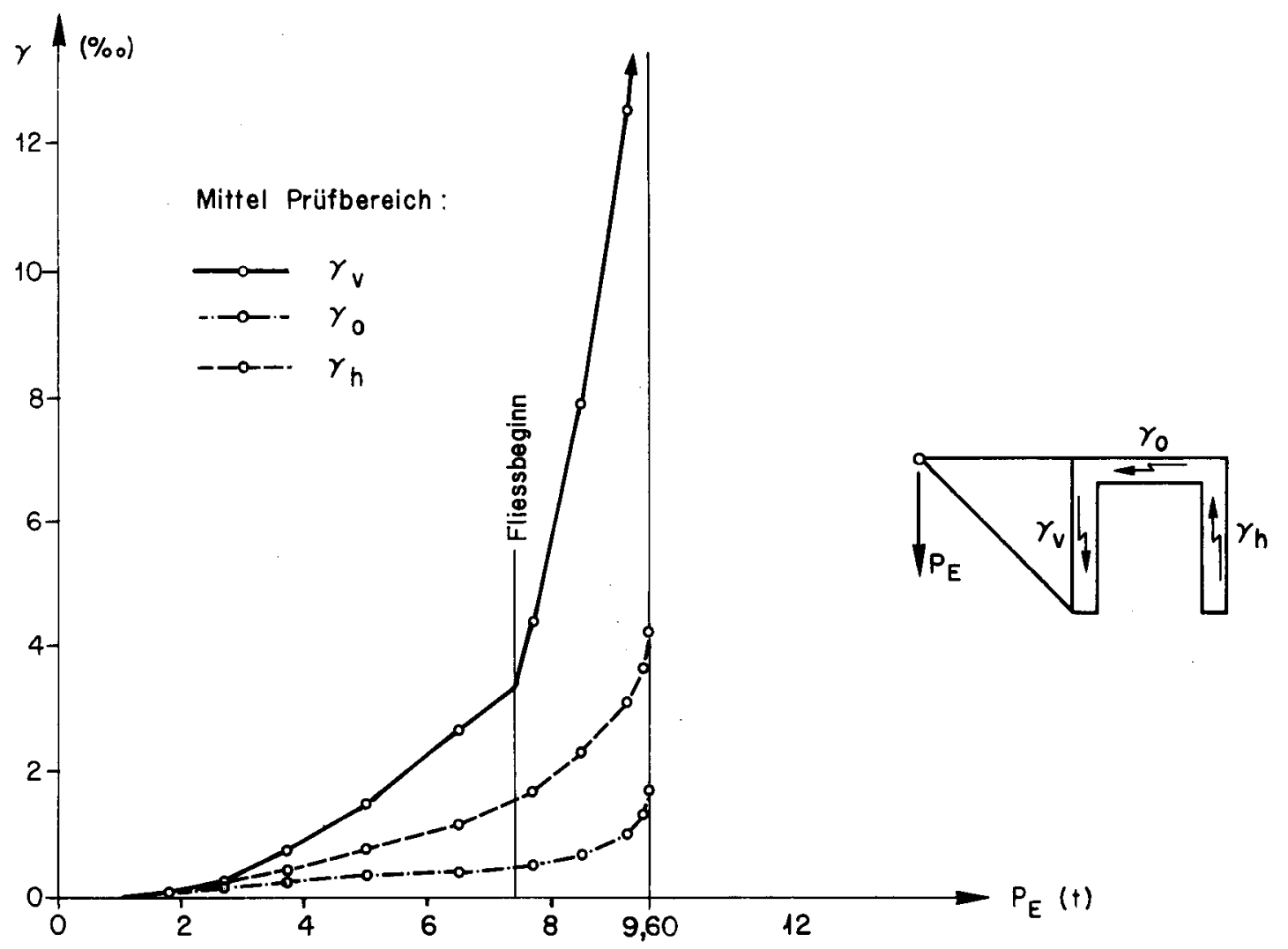

Bild 42: Schiebungen $\gamma$ Balken $\mathrm{TW}_{4}$ in Funktion der Belastung 


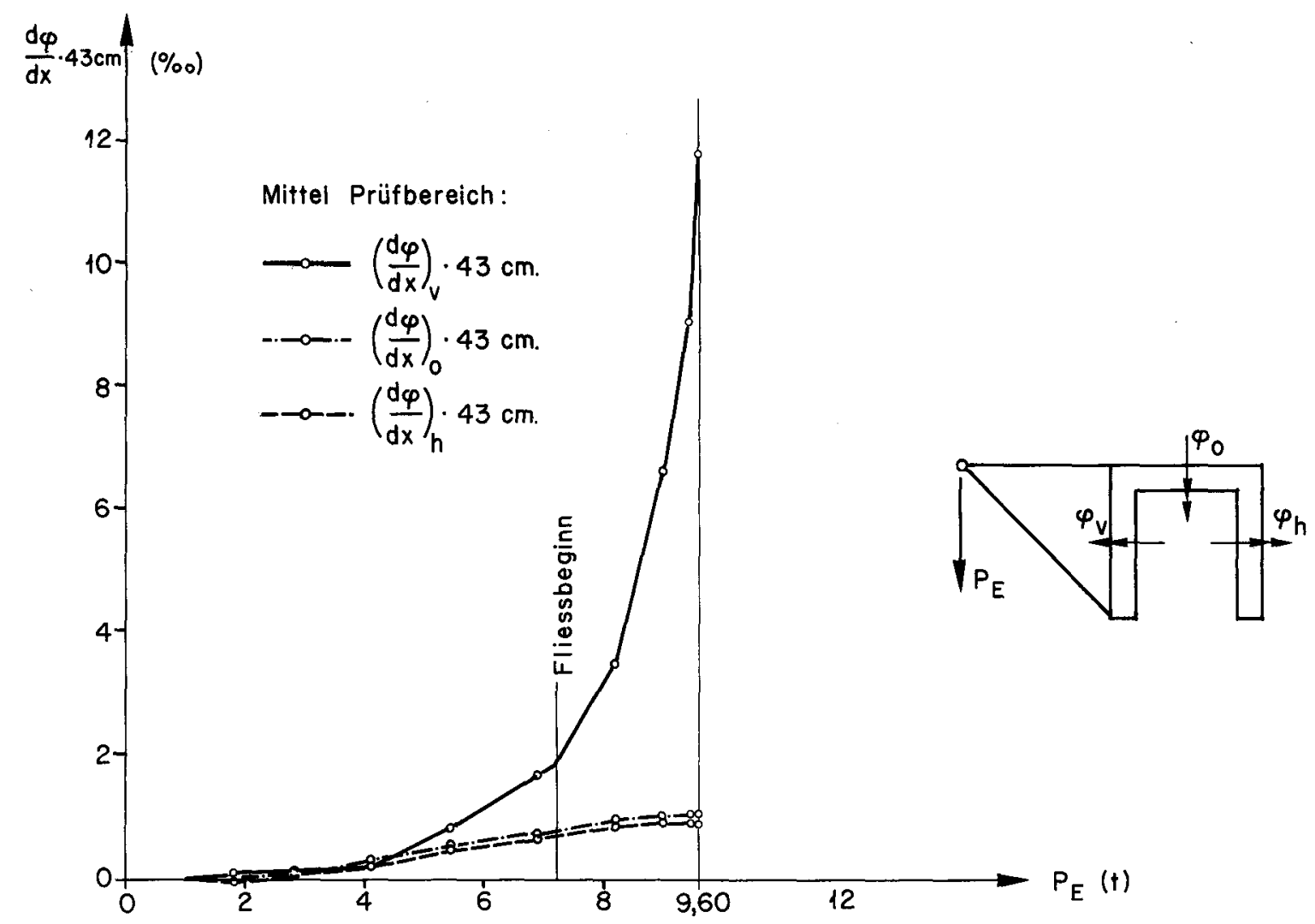

Bild 43: Krümmungen $\frac{d \varphi}{d x}$ Balken $T_{5}$ in Funktion der Belastung

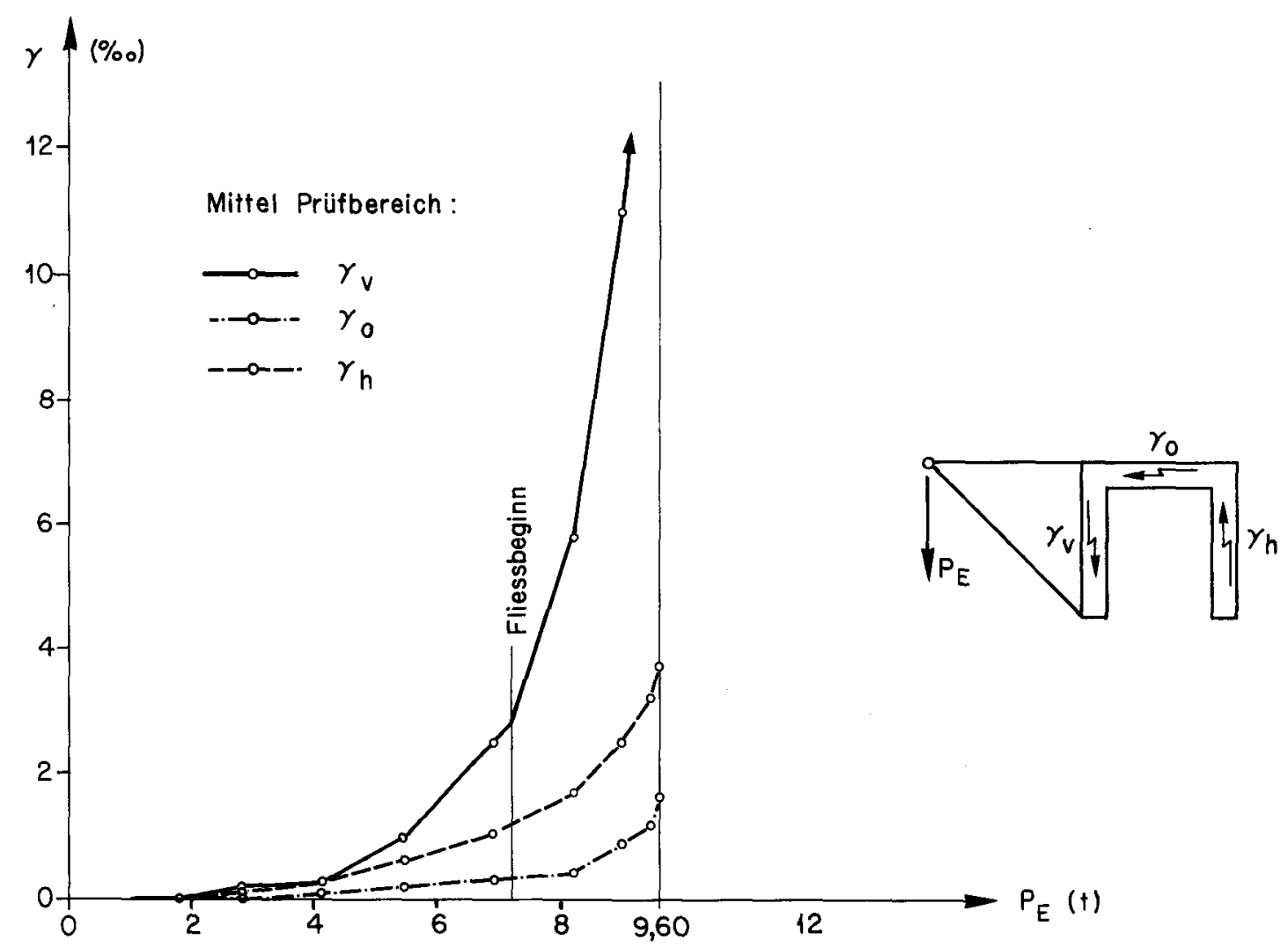

Bild 44: Schiebungen $\gamma$ Balken $T_{W}$ in Funktion der Belastung 


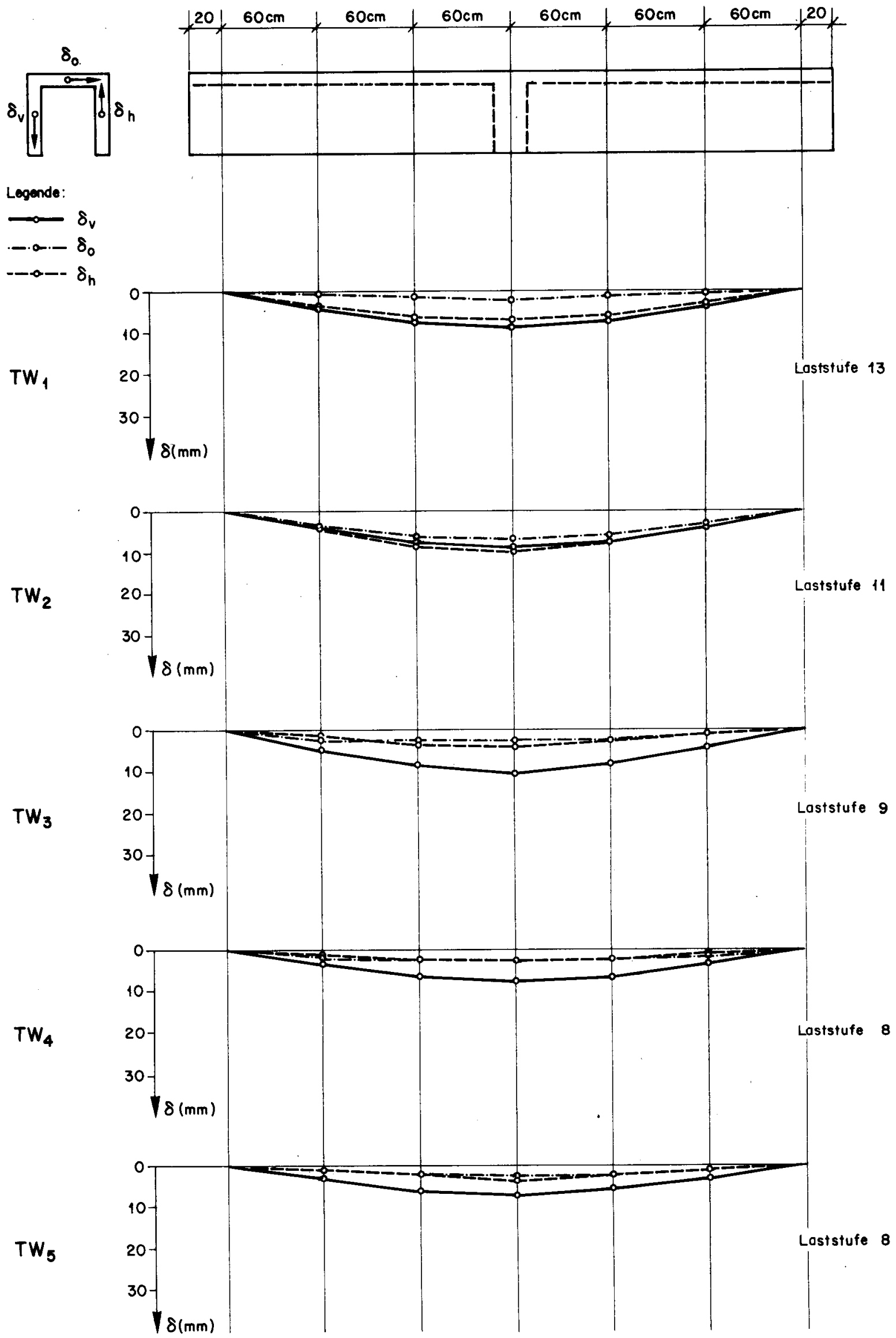

Bild 45: Durchbiegungen Balken $T W_{1}$ bis $T W_{5}$ vor Fliessbeginn 


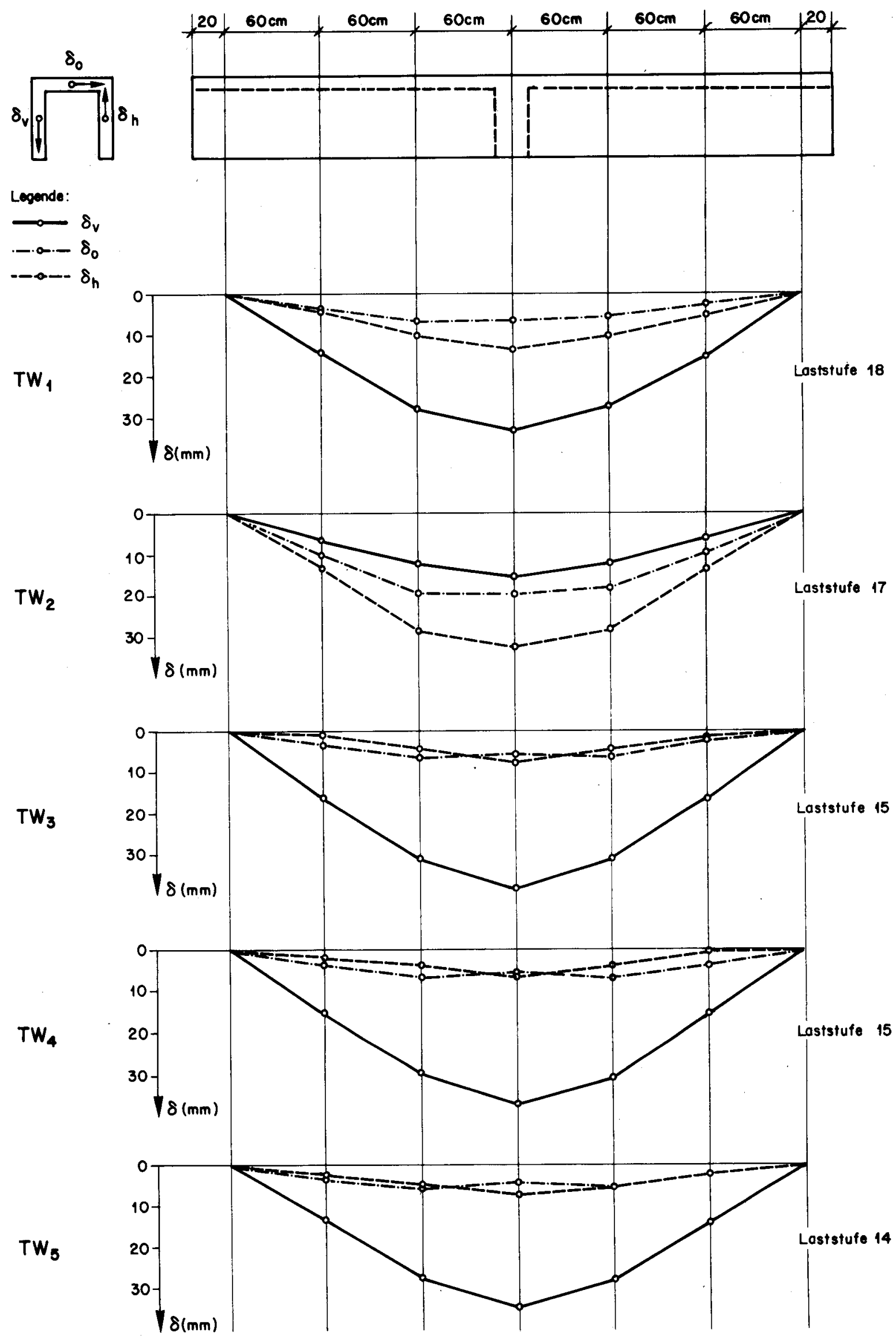

Bild 46: Durchbiegungen Balken TW 


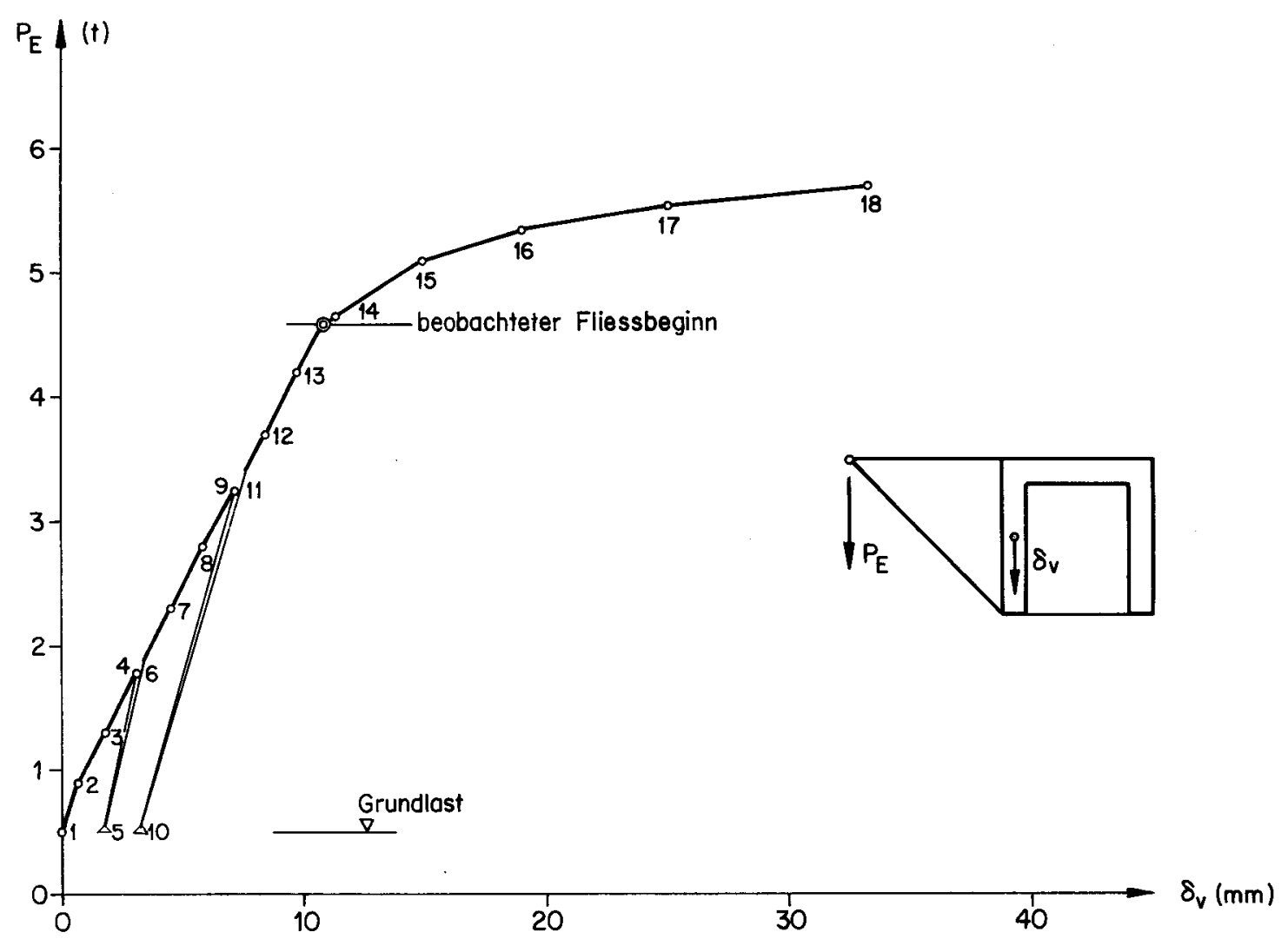

Bild 47: Durchbiegung $\delta_{v}$ in Balkenmitte von TW

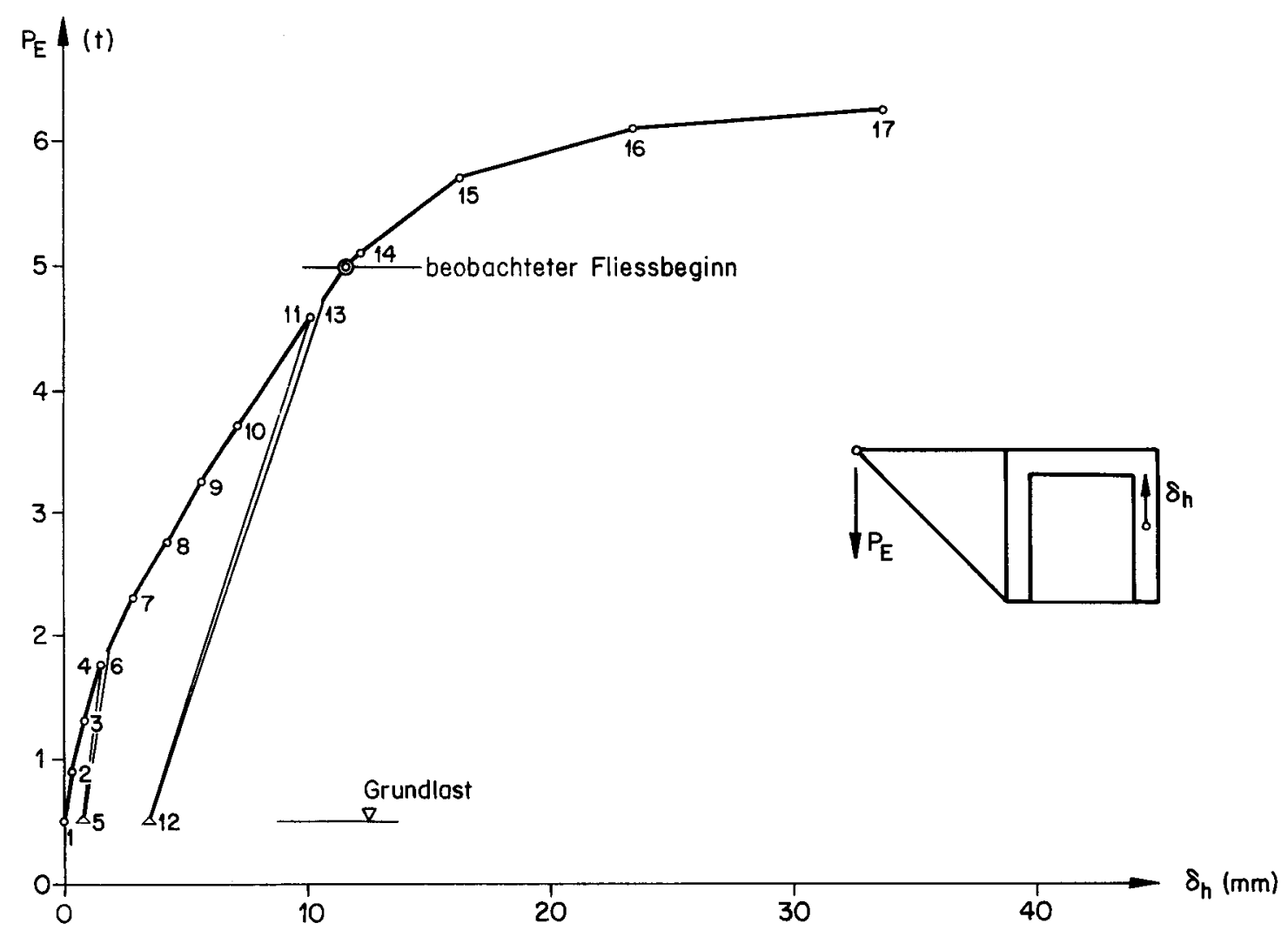

Bild 48: Durchbiegung $\delta_{h}$ in Balkenmitte von $\mathrm{TW}_{2}$ 


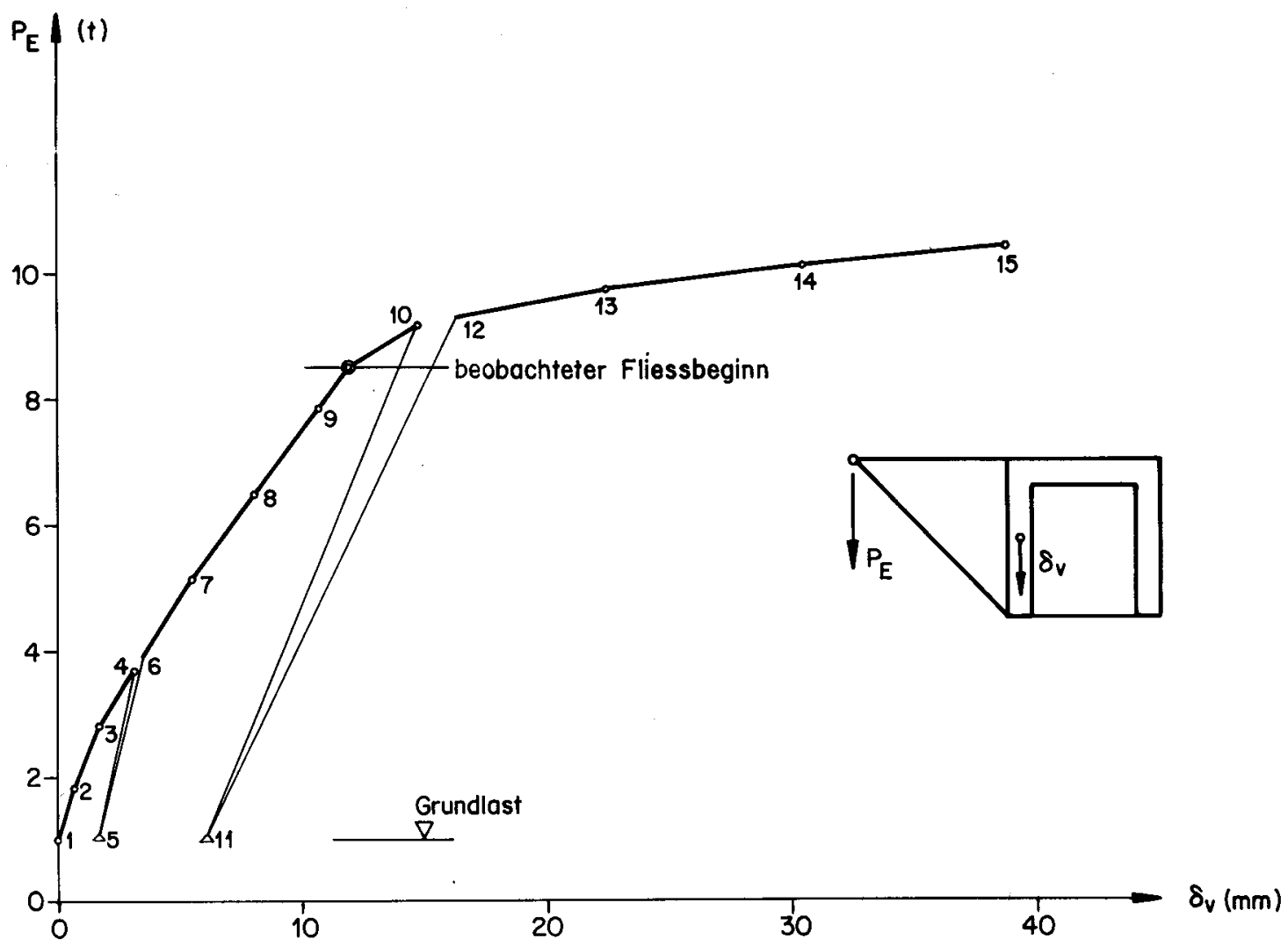

Bild 49: Durchbiegung $\delta_{v}$ in Balkenmitte von $\mathrm{TW}_{3}$

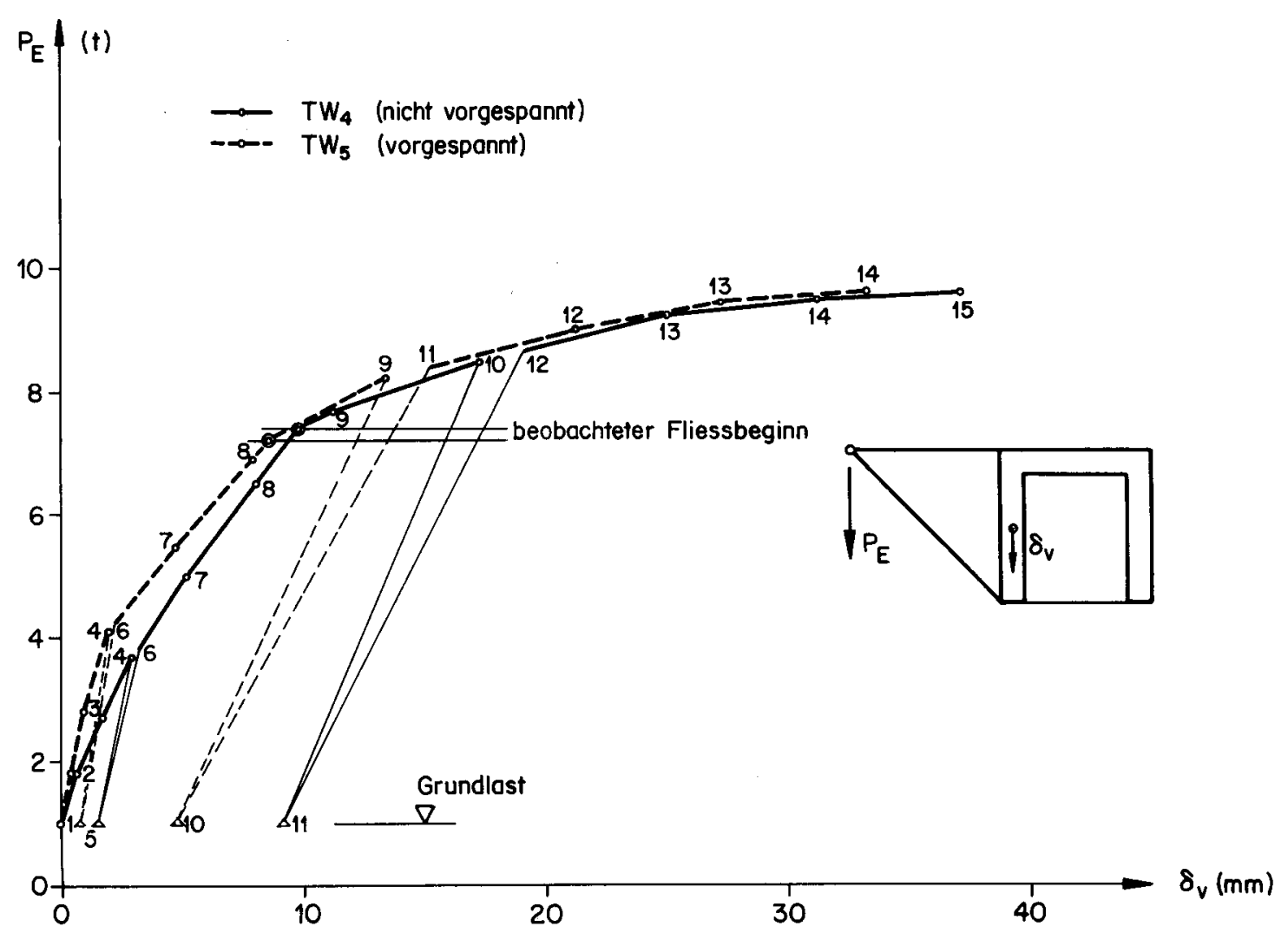

Bild 50: Durchbiegung $\delta_{v}$ in Balkenmitte von $\mathrm{TW}_{4}$ und $\mathrm{TW}_{5}$ 


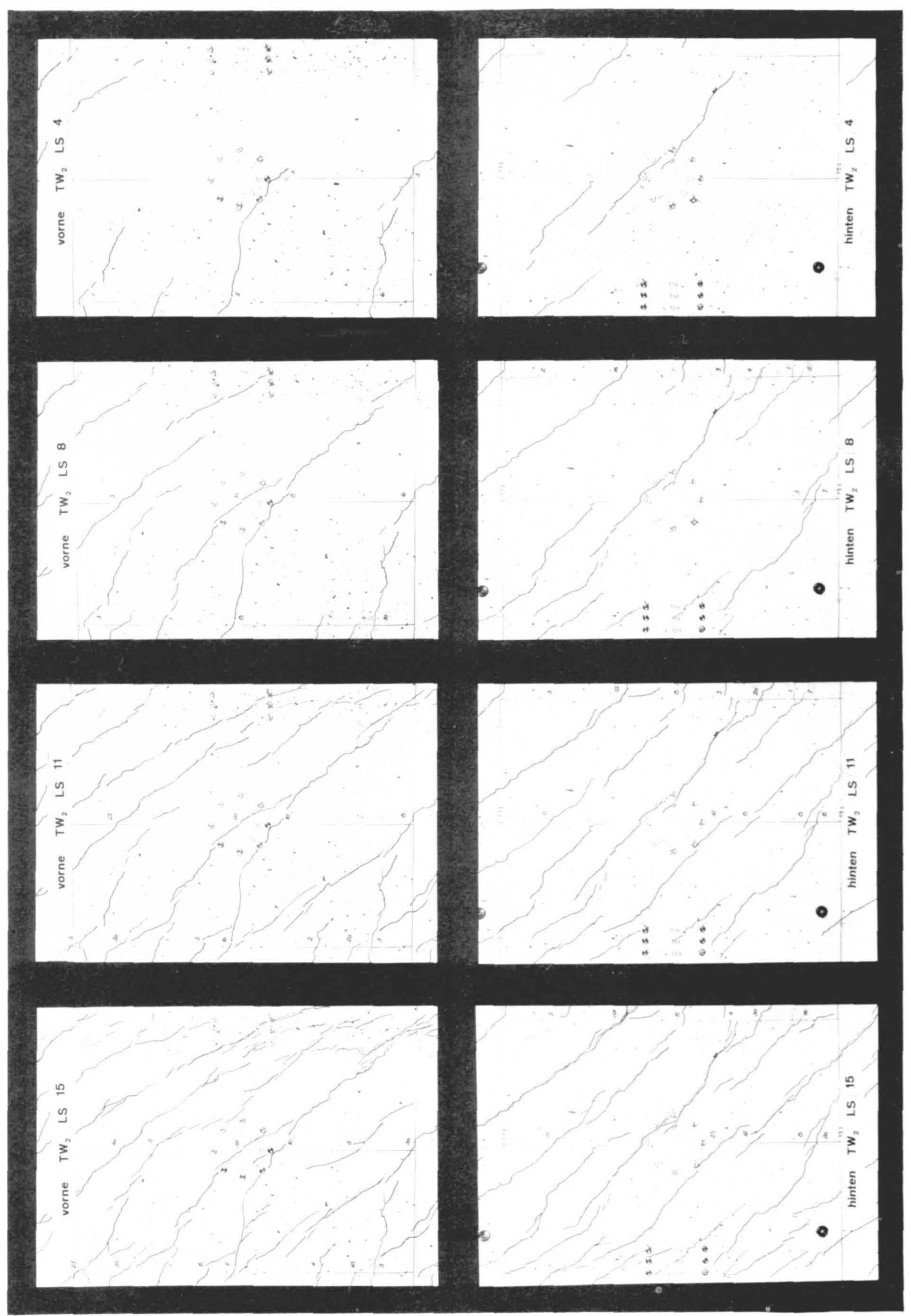

Zahlen bedeuten Rissweiten in 1/100 mm

Bild 51: Rissverlauf vorne und hinten Balken $\mathrm{TW}_{2}$ 


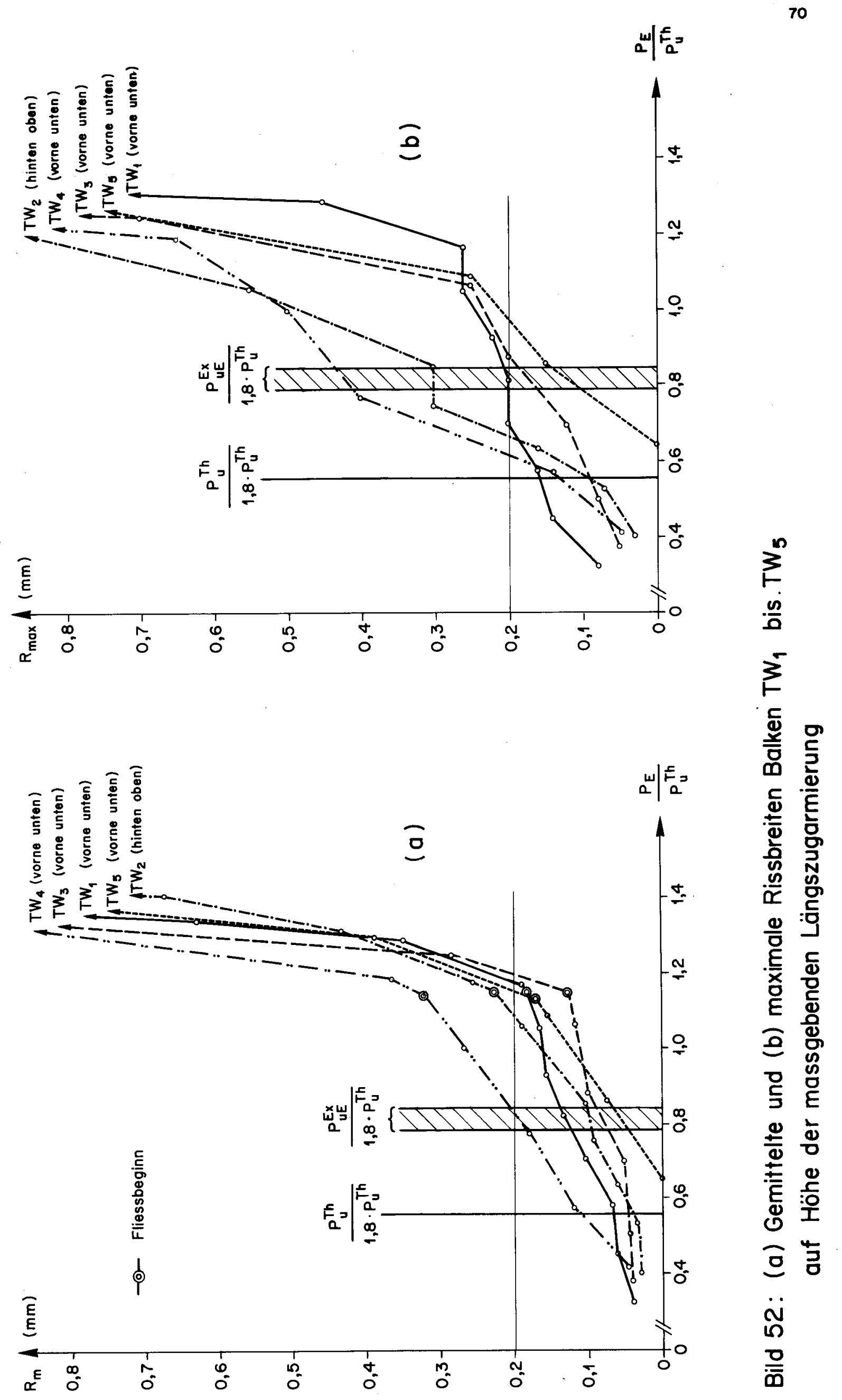


Die Stabtheorie, wie sie im Abschnitt 2.3 dieses Berichtes dargestellt ist, setzt voraus, dass die Erhaltung der Querschnittsform garantiert ist. Andererseits ist die Annahme möglich, dass sich der Querschnitt frei verformen kann. Dies ist der Fall, wenn neben der Vernachlässigung der Plattenwirkung (Biege- und Torsionswiderstand) die Einzelscheiben des Querschnittes gelenkig miteinander verbunden sind. Solche Tragwerke werden als Gelenkfaltwerke oder einfach als Faltwerke bezeichnet.

Diese beiden Theorien, die Stabtheorie und die Faltwerktheorie, werden einander im folgenden Bild gegenübergestellt.

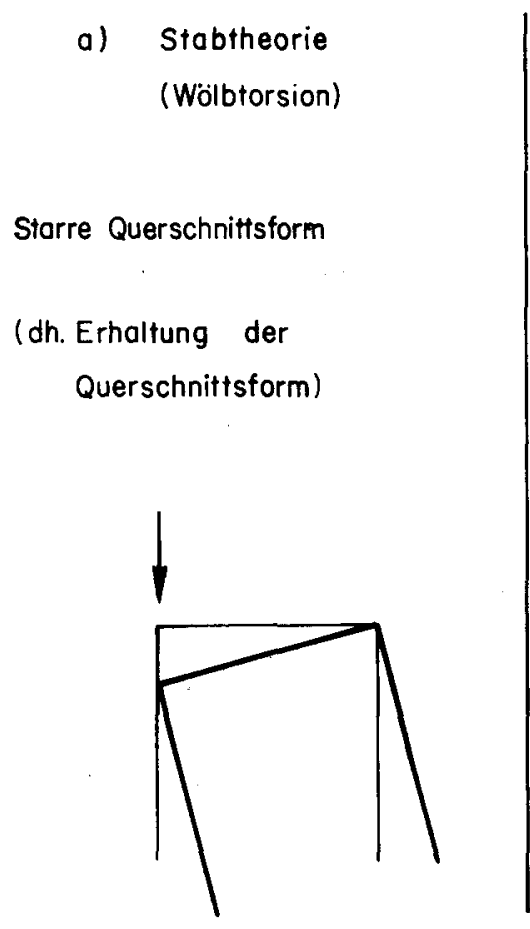

b) Faltwerktheorie

\section{Gelenkige Verbindung der Einzelscheiben}

(dh. keine Erhaltung der Querschnittsform )

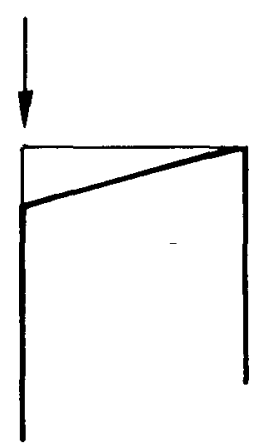

Bild A1: Gegenüberstellung der Stab-und Faltwerktheorie

Im allgemeinen erhält man nach diesen beiden Theorien unterschiedliche Resultate. Für sogenannte Dreischeiben-Querschnitte, wie sie beispielsweise die untersuchten Balken $T W_{1}$ bis $T W_{5}$ aufweisen, gilt jedoch folgende Besonderheit.

Wird die Plattenwirkung (Biege- und Torsionswiderstand) der Querschnittscheiben vernachlässigt, so liefern für Dreischeiben-Querschnitte sowohl die Faltwerk- als auch die Stabtheorie dieselben Resultate. Die Frage, ob die Querschnittsform erhalten bleibt oder nicht, spielt nämlich bei Beschränkung auf die Theorie 1. Ordnung keine Rolle, da die Zerlegung einer beliebigen last in drei Scheibenebenen eindeutig, d.h. statisch bestimmt ist. Ist die Belastung nicht von vorne herein in form von Kantenbzw. Scheibenlasten gegeben, so muss die Einleitung der Lasten in diese Scheiben ge- 
währleistet sein. Die Einleitung beliebiger Lasten kann z.B. durch Querträger, Queraussteifungen oder durch lokale Plattenwirkung der Querschnittscheiben erzielt werden. Im letzteren Falle wird die Plattenwirkung wohl für die Einleitung der Lasten in die Querschnittscheiben herangezogen, man vernachlässigt sie aber für die eigentliche Berechnung des Faltwerkes.

Es soll hier eine Berechnungsmethode hergeleitet werden, die auf der Faltwerktheorie basiert. Es gelten die für Gelenkfaltwerke üblichen Voraussetzungen:

1) Kleine Querschnittsabmessungen im Vergleich zur Länge ( + Berechnung der Einzelscheibe nach Balkentheorie)

2) Formulierung des Gleichgewichtes am unverformten System (Theorie 1. Ordnung)

3) Vernachlässigung der Plattenwirkung (Biegeund Torsionswiderstand) der Einzelscheiben

4) Gelenkige Verbindung der Einzelscheiben durch Scharniere

5) Gleiche Lagerung aller Einzelscheiben (entsprechend Stabtheorie)

Als Modell für die Wirkungsweise eines Stahlbeton-Faltwerkes wird ein räumliches Fachwerk angenommen. In ihm bilden die Längsstäbe die Gurtungen, die Bügel die Pfosten und die Betondiagonalen die Streben. Ausserdem denkt man sich zwecks einfacher mathematischer Behandlung die Längseisen in den Kanten des Faltwerkes konzentriert. Das folgende Bild zeigt einen Ausschnitt aus einem derartigen Faltwerk. Die Wirkungsweise als Fachwerk ist aus der Scheibe $i-1$ ersichtlich.

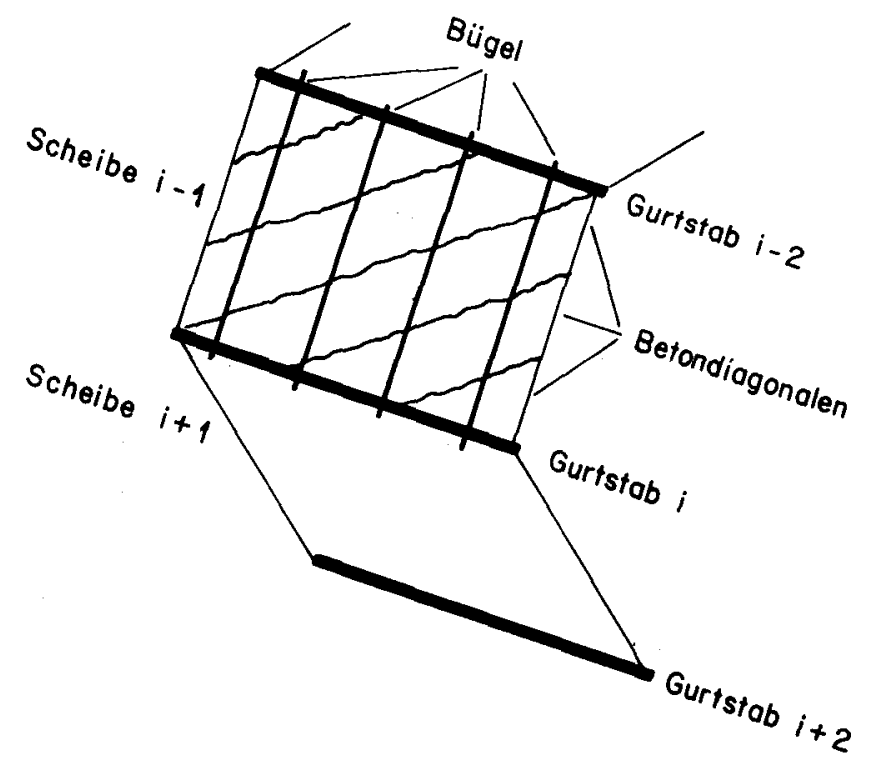

Bild A2: Idealisierung eines Stahlbeton-Faliwerkes 
Für die Beschreibung des Kräftespiels in einer Einzelscheibe wird wie in Abschnitt 2.3.2 ein Fachwerkmodell mit variabler Diagonalenneigung angenommen.

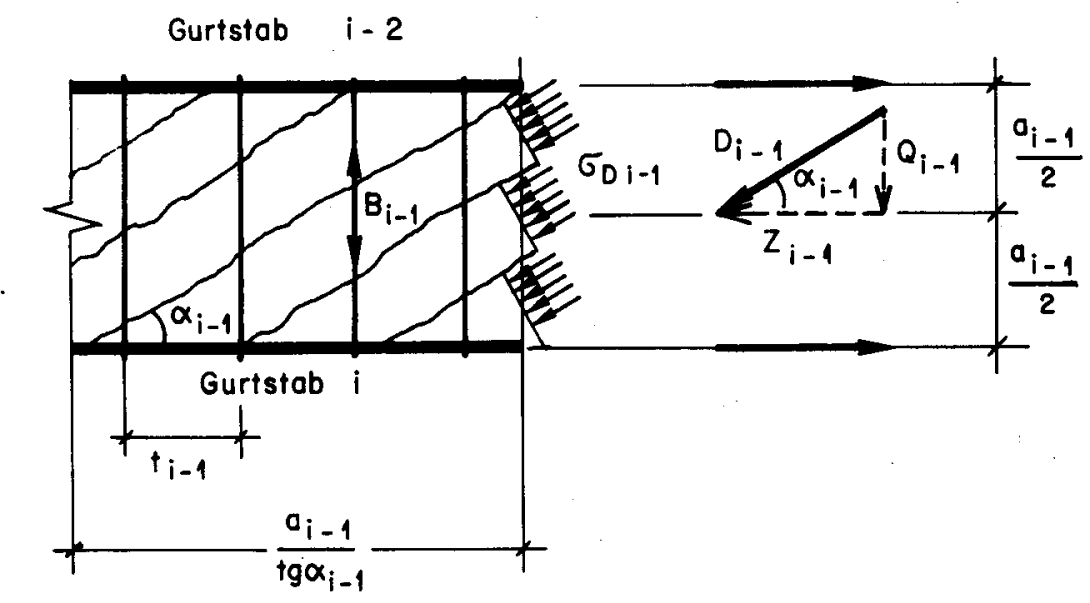

\section{Bild A3: Wirkungsweise einer Einzelscheibe}

Bei Annahme gleichmässig über die Scheibe verteilter Betondruckspannungen $\sigma_{D} i-1$ resultiert aus der Schubbeanspruchung $Q_{i-1}$ eine in Mitte der Scheibe wirkende Längskraft $z_{i-1}$.

Die Annahme einer solchen Druckspannungsverteilung bedingt auch eine gleichmässige Beanspruchung der Bügel im dargestellten Scheibenabschnitt, was zu folgenden Gleichgewichtsbedingungen führt:

$$
\left.\begin{array}{l}
Q_{i-1}=B_{i-1} \cdot \frac{a_{i-1}}{t_{i-1}} \cdot \frac{1}{\operatorname{tg} \alpha_{i-1}} \\
Z_{i-1}=Q_{i-1} \cdot \frac{1}{\operatorname{tg} \alpha_{i-1}}=B_{i-1} \cdot \frac{a_{i-1}}{t_{i-1}} \cdot \frac{1}{\operatorname{tg}^{2} \alpha_{i-1}}
\end{array}\right\}
$$

Der Geltungsbereich dieses Fachwerkmodells ist auf einen gewissen Neigungsbereich der Betondiagonalen beschränkt. Wie den Versuchsberichten [1] bis [5] éntnommen werden kann, bewegt sich die Diagonalenneigung in den Grenzen:

$$
0,5 \leq\left|t_{g \alpha_{i-1}}\right| \leq 2,0
$$

Für die weitere Behandlung wird das Faltwerk längs den Kanten aufgetrennt und in Einzelscheiben zerlegt. Dabei werden, wie aus Bild A4 hervorgeht, die in den Kanten konzentrierten Gurtstäbe auf beide benachbarten Scheiben aufgeteilt.

Auf eine solche Einzelscheibe wirken die Kantenschubkräfte, die entsprechend den gelösten Bindungen énzuführen sind sowie die aus der Scheibenbelastung resultierenden Schnittkräfte. Diese lassen sich bei den gemachten Voraussetzungen nach der Balkenstatik ermitteln. Beschränkt men sich auf statisch bestimmt gelagerte Einzelscheiben, so benötigt man zur Bestimmung der Scheiben-Schnittkräfte lediglich die Gleichgewichtsbedingungen, d.h. die Schnittkraftverteilung ist unabhängig von irgendwelchem Stoffgesetz. 
Das Kräftespiel an einer Einzelscheibe ist in Bild A4 dargestellt.

Innere Kräfte

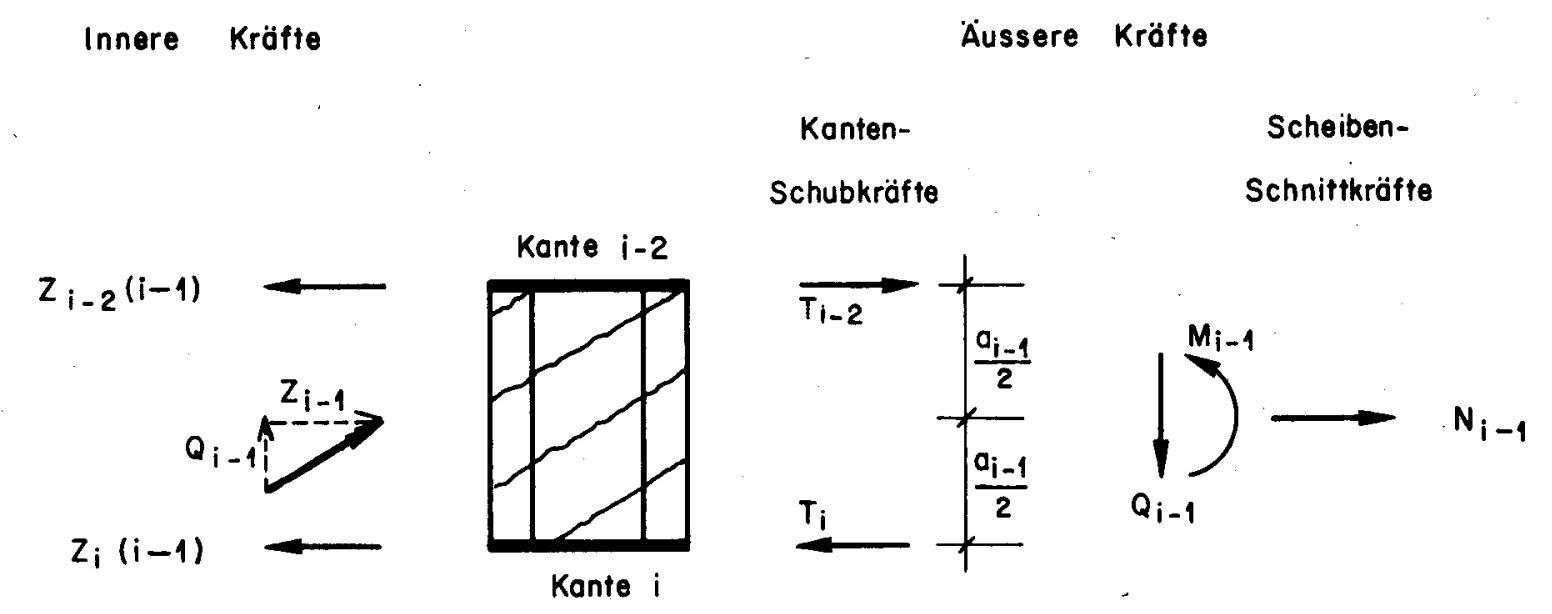

Das

Bild A4: Kräftespiel an einer Einzelscheibe

Die im Gurtstab $i$ wirkende kraft $z_{i}$ setzt sich aus zwei Anteilen zusammen. Der eine. Anteil $Z_{i}(i-1)$ lässt sich mit Hilfe der Gleichgewichtsbedingungen an der Scheibe $i-1$ (Bild $A 4$ ) bestimmen.

$$
\left.\begin{array}{rl}
z_{i}(i-1) & =\frac{N_{i-1}}{2}+\frac{M_{i-1}}{a_{i-1}}+\frac{z_{i-1}}{2}-T_{i}= \\
& =\frac{N_{i-1}}{2}+\frac{M_{i-1}}{a_{i-1}}+\frac{Q_{i-1}}{2 \cdot \operatorname{tg} \alpha_{i-1}}-T_{i}
\end{array}\right\}
$$

Den anderen Anteil $z_{i}(i+1)$ erhält man aus derselben Betrachtung der Scheibe $i+1$.

$$
\left.\begin{array}{rl}
z_{i}(i+1) & =\frac{N_{i+1}}{2}-\frac{M_{i+1}}{a_{i+1}}+\frac{z_{i+1}}{2}+T_{i}= \\
& =\frac{N_{i+1}}{2}-\frac{M_{i+1}}{a_{i+1}}+\frac{Q_{i+1}}{2 \cdot \operatorname{tg} \alpha_{i+1}}+T_{i}
\end{array}\right\}
$$

Die Addition dieser beiden Anteile liefert die gesamte im Gurtstab i wirkende Kraft $z_{i}$. Wie aus der Gleichung für $z_{i} z u$ entnehmen ist, haben die Kantenschübe dank der Konzentration der Gurtstäbe in den Faltwerkkanten keinen Einfluss auf die Gurtstabkräfte. 


$$
\begin{aligned}
& z_{i}=\frac{1}{2} \cdot\left(N_{i-1}+N_{i+1}\right)+\left(\frac{M_{i-1}}{a_{i-1}}-\frac{M_{i+1}}{a_{i+1}}\right)+ \\
& \quad+\frac{1}{2} \cdot\left(\frac{Q_{i-1}}{\operatorname{tg} \alpha_{i-1}}+\frac{Q_{i+1}}{\operatorname{tg} \alpha_{i+1}}\right) \\
& B_{i-1} \cdot \frac{a_{i-1}}{t_{i-1}} \cdot \frac{1}{\operatorname{tg} \alpha_{i-1}}=Q_{i-1} \\
& B_{i+1} \cdot \frac{a_{i+1}}{t_{i+1}} \cdot \frac{1}{\operatorname{tg} \alpha_{i+1}}=Q_{i+1}
\end{aligned}
$$

Der Vollständigkeit halber sind hier noch die aus (A1) abgeleiteten Gleichungen für die Bügelkräfte $B_{i-1}$ und $B_{i+1}$ aufgeführt. Damit sind alle Gleichgewichtsbedingungen zusammengestellt, die zur Erfassung des beschriebenen Faltwerkmodelles gebraucht werden.

Diese Beziehungen können zum Beispiel auf die Versuchsbalken TW bis $_{1} W_{5}$ angewandt werden. Bei Beschränkung auf die wesentliche Beanspruchung infolge der Einzellast P erhält man aus den dargestellten Scheibenlasten

\section{Belostung}

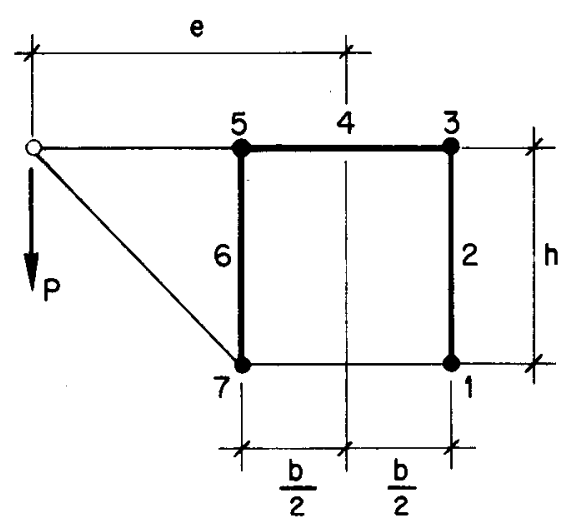

Scheibenlasten

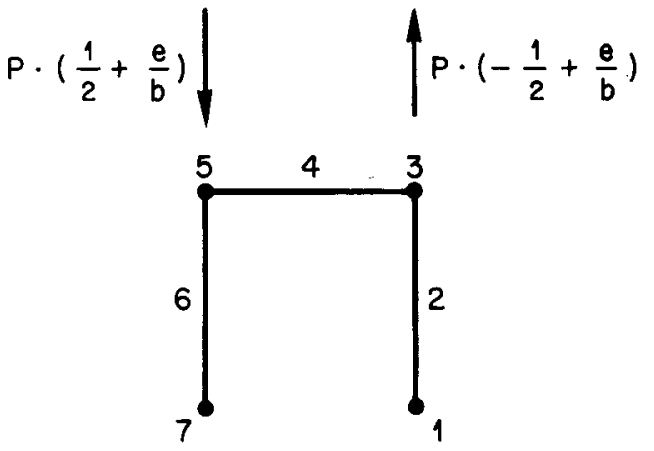

Ungerade Numerierung: Kanten

Gerade Numerierung : Scheiben

Bild A5: Zerlegung der Belastung in Scheibenlasten (Beispiel) 
folgende Scheiben-Schnittkräfte im Querschnitt A-A der Versuchskörper (vgl. Bild 14).

$$
\begin{array}{ll}
Q_{2}=-\frac{P}{2} \cdot\left(-\frac{1}{2}+\frac{e}{b}\right) & Q_{6}=-\frac{P}{2} \cdot\left(\frac{1}{2}+\frac{e}{b}\right) \\
M_{2}=\frac{P \cdot 1}{8} \cdot\left(-\frac{1}{2}+\frac{e}{b}\right) & M_{6}=\frac{P \cdot 1}{8} \cdot\left(\frac{1}{2}+\frac{e}{b}\right)
\end{array}
$$

Daraus ergeben sich mit den Gleichungen (A5) die gesuchten Bügel- und Gurtstabkräfte.

$$
\begin{aligned}
& B_{2} \cdot \frac{h}{t_{B}} \cdot \frac{1}{\operatorname{tg} \alpha_{2}}=Q_{2}=-\frac{P}{2} \cdot\left(-\frac{1}{2}+\frac{e}{b}\right) \\
& B_{4} \cdot \frac{b}{t_{B}} \cdot \frac{1}{\operatorname{tg} \alpha_{4}}=Q_{4}=0 \\
& B_{6} \cdot \frac{h}{t_{B}} \cdot \frac{1}{\operatorname{tg} \alpha_{6}}=Q_{6}=-\frac{P}{2} \cdot\left(\frac{1}{2}+\frac{e}{b}\right) \\
& z_{1}=\frac{P}{4} \cdot\left(-\frac{1}{2}+\frac{e}{b}\right) \cdot\left(-\frac{1}{2 h}-\frac{1}{\operatorname{tg} \alpha_{2}}\right) \\
& z_{3}=\frac{P}{4} \cdot\left(-\frac{1}{2}+\frac{e}{b}\right) \cdot\left(\frac{1}{2 h}-\frac{1}{\operatorname{tg} \alpha_{2}}\right) \\
& z_{5}=\frac{P}{4} \cdot\left(\frac{1}{2}+\frac{e}{b}\right) \cdot\left(-\frac{1}{2 h}-\frac{1}{\operatorname{tg} \alpha_{6}}\right) \\
& z_{7}=\frac{P}{4} \cdot\left(\frac{1}{2}+\frac{e}{b}\right) \cdot\left(\frac{1}{2 h}-\frac{1}{\operatorname{tg} \alpha_{6}}\right)
\end{aligned}
$$

Dieselben Resultate erhält man auch nach den im Abschnitt 2.3.2 angegebenen Beziehungen der Stabtheorie. Dazu braucht man lediglich die aus $P$ resultierenden Schnittkräfte $Q_{z}, M_{z}, T_{w}$ und $M_{w}$ im Querschnitt $A-A$ (siehe Bild 14 rechts) in die Gleichungen (13) einzusetzen.

Bei Beschränkung auf unterarmierte Stahlbeton-Träger, bei denen der Beton für das Versagen nicht massgebend ist, kann ein duktiles Bruchverhalten vorausgesetzt werden. Dies ermäglicht die Anwendung der Plastizitätstheorie, welche mit den folgenden Annahmen arbeitet.
1) Starr-plastisches Materialverhalten
2) Proportionale Steigerung der Belastung bis zum Bruch

Die zweite Voraussetzung bedeutet, dass alle Lasten proportional bis zum Bruch um denselben Traglastfaktor $\lambda$ erhöht werden. Bei statisch bestimmt gelagerten Faltwerken führt eine proportionale Steigerung der Belastung auch zu einer proportionalen Steigerung der Scheiben-Schnittkräfte.

$$
\left\{\begin{array}{c}
N_{i-1} \\
M_{i-1} \\
Q_{i-1}
\end{array}\right\} \longrightarrow\left\{\begin{array}{l}
\lambda \cdot N_{i-1} \\
\lambda \cdot M_{i-1} \\
\lambda \cdot Q_{i-1}
\end{array}\right\}
$$


In schubbeanspruchten Stahlbeton-Scheiben stellt sich innerhalb gewisser Gnenzen aufgrund der Rissverzahnung die Neigung der Betondiagonalen so ein, dass sowohl Längsals auch Bügelarmierung zum Fliessen kommen. Dies heisst mit Blick auf das beschriebene Faltwerk-Modell, dass beim Fliessen eines Gurtstabes im allgemeinen auch die Bügel der beiden benachbarten Scheiben ihre Fliessgrenze erreichen.

Damit hat man bereits einen Querschnittsmechanismus erreicht, weil die Scheiben des Faltwerkes voraussetzungsgemäss gelenkig miteinander verbunden sind.

Es gilt:

Bei einem Querschnittsmechanismus müssen im allgemeinen ein Gurtstab sowie die Bügel der beiden benachbarten Scheiben fliessen.

Zu erwähnen ist noch, dass bei einem statisch bestimmt gelagerten Faltwerk der Mechanismus des Tragwerkes durch den Mechanismus eines einzigen Querschnittes gegeben ist (Proportionale Steigerung der Belastung = Proportionale Steigerung der Schnittkräfte). Hingegen führt ein solcher Querschnittsmechanismus bei einem statisch unbestimmt gelagerten System nicht zwangsweise zu einem Mechanismus des Tragwerkes.

Falls der Gurtstab $i$ sowie die Bügel der Scheiben $i-1$ und $i+1$ für ein Versagen massgebend sind, so ergeben sich aus (A5), wo die Kräfte $z_{i}, B_{i-1}$ und $B_{i+1}$ durch ihre Fliesskräfte $Z_{f i}, B_{f i-1}$ und $B_{f i+1}$ zu ersetzen sind, und aus $(A 6)$ folgende Beziehungen.

$$
\begin{aligned}
& \left.\begin{array}{l}
\frac{1}{\operatorname{tg} \alpha_{i-1}}=\lambda \cdot Q_{i-1} \cdot\left(B_{f} i-1 \cdot \frac{a_{i-1}}{t_{i-1}}\right)^{-1} \\
\frac{1}{\operatorname{tg} \alpha_{i+1}}=\lambda \cdot Q_{i+1} \cdot\left(B_{f} i+1 \cdot \frac{a_{i+1}}{t_{i+1}}\right)^{-1}
\end{array}\right\} \\
& \left.\begin{array}{l}
z_{f i}=\lambda \cdot\left(\frac{1}{2} \cdot\left(N_{i-1}+N_{i+1}\right)+\left(\frac{M_{i-1}}{a_{i-1}}-\frac{M_{i+1}}{a_{i+1}}\right)\right]+ \\
+\frac{\lambda^{2}}{2} \cdot\left[\left(Q_{i-1}\right)^{2} \cdot\left(B_{f} i-1 \cdot \frac{a_{i}-1}{t_{i-1}}\right)^{-1}+\left(Q_{i+1}\right)^{2} \cdot\left(B_{f} i+1 \cdot \frac{a_{i+1}}{t_{i+1}}\right)^{-1}\right]
\end{array}\right\}
\end{aligned}
$$

Die Gleichung (A9) liefert die Lösung für die Traglast bzw. den Traglastfaktor $\lambda$. Mit den Beziehungen (A8) können die beiden Diagonalneigungen $\alpha_{i-1}$ und $\alpha_{i+1}$ bestimmt werden. Fällt einer dieser Werte aus dem in (A2) angegebenen Bereich, so verlieren die Beziehungen (AP) bis (A9) ihre Gültigkeit, da sich die Diagonalenneigung nicht beliebig variieren lässt. Bei Einhaltung der entsprechenden Grehzen sind neben dem beschriebenen allgemeinen Fall noch folgende Spezialfälle denkbar. 
Gurtstab i

Scheibe i-1

Scheibe $i+1$

Bruchart

\begin{tabular}{|c|c|c|c|}
\hline Fliessen & $\begin{array}{c}\text { Bügelfliessen } \\
0,5 \leq\left|\dagger g \alpha_{i-1}\right| \leq 2,0\end{array}$ & $\begin{array}{c}\text { Bügelfliessen } \\
0,5 \leq \mid \operatorname{tg} \alpha_{i+1} \leq 2,0\end{array}$ & \multirow{3}{*}{$\begin{array}{c}\text { Biege- } \\
\text { Schubbrüche }\end{array}$} \\
\hline Fliessen & $\begin{array}{c}\text { Bügelfliessen } \\
0,5 \leq\left|\operatorname{tg} \alpha_{i-1}\right| \leq 2,0\end{array}$ & $\begin{array}{c}\text { Kein Bügelfliessen } \\
\mid \operatorname{tg} \alpha_{i+1}=2,0 \text { oder } Q_{i+1} \rightarrow 0\end{array}$ & \\
\hline Fliessen & $\begin{array}{c}\text { Kein Bügelfliessen } \\
\left|\operatorname{tg} \alpha_{i-1}\right|=2,0 \text { oder } Q_{i-1} \rightarrow 0\end{array}$ & $\begin{array}{c}\text { Bügelfliessen } \\
0,5 \leq \mid \operatorname{tg} \alpha_{i+1 \mid \leq 2,0}\end{array}$ & \\
\hline Fliessen & $\begin{array}{c}\text { Kein Bügelfliessen } \\
\left|\operatorname{tg} \alpha_{i-1}\right|=2,0 \text { oder } Q_{i-1} \rightarrow 0\end{array}$ & $\begin{array}{c}\text { Kein Bügelfliessen } \\
\operatorname{tg} \alpha_{i+1}=2,0 \text { oder } Q_{i+1} \rightarrow 0\end{array}$ & Biegebrüche \\
\hline Kein Fliessen & $\begin{array}{l}\text { Bügelfliessen } \\
\mid \operatorname{tg} \alpha_{i-1}=0,5\end{array}$ & $\begin{array}{l}\text { Bügelfliessen } \\
\mid t g \alpha_{i+1 \mid}=0,5\end{array}$ & \multirow{3}{*}{ Schubbrüche } \\
\hline Kein Fliessen & $\begin{array}{l}\text { Bügelfliessen } \\
\left|\operatorname{tg} \alpha_{i-1}\right|=0,5\end{array}$ & Kein Bügelfliessen & \\
\hline Kein Fliessen & Kein Bügelfliessen & $\begin{array}{l}\text { Bügelfliessen } \\
\operatorname{tg} \alpha_{i+1}=0,5\end{array}$ & \\
\hline
\end{tabular}

Für jede dieser Brucharten erhält man die Traglast auf gleiche Weise wie im allgemeinen Fall, wo ein Gurtstab und die Bügel der beiden benachbarten Scheiben fliessen. Auf eine weitere Behandlung dieser Spezialfälle wird deshalb verzichtet.

Abschliessend sei noch auf die Versuchsbalken TW, bis TW 5 hingewiesen. Die Belastung, wie sie Bild A5 zeigt, führt zu einem Spezialfall, da die Scheibe 4 nicht auf Schub beansprucht wird. Aus diesem Grunde müssen nach dem obigen Tableau beim Fliessen des Gurtstabes 3 nur die Bügel der Scheibe 2 ins Fliessen kommen. In diesem Zusammenhang ist zu erwähnen, dass die Gleichung ( $A g$ ) auch auf alle jene Fälle angewandt werden kann, wo entweder die Schubbeanspruchung beider Scheiben null ist oder wo die Bügel in der einen Scheibe fliessen und die Schubbeanspruchung der anderen Scheibe verschwindet. Die Gleichung ( $A 9$ ) gilt demnach auch für die Versuchsbalken und führt zu denselben Resultaten wie die in Abschnitt 2.3 .2 angegebenen Beziehungen (14b) und $(15 b)$. 